Alessandra Teodoro Neves

\title{
APLICAÇÃO DOS MODELOS PARAMÉTRICOS ARMAV E ARV NA IDENTIFICAÇÃO MODAL DE SISTEMAS MECÂNICOS
}

\begin{abstract}
Tese apresentada à Escola de Engenharia de São Carlos da Universidade de São Paulo, como parte dos requisitos para a obtenção do Título de Doutor em Engenharia Mecânica.
\end{abstract}

Orientador: Prof. Titular Eduardo Morgado Belo Co-Orientador: Prof. Dr. MáRIo Francisco Mucheroni 
Aos meus amores: meus pais, Jesus e Maria por simplesmente tudo...; meu marido Francisco, amor e companheiro, pelo incentivo, compreensão e paciência; minha filha, meu presente de Deus, minha preciosidade... Ana Laura. 


\section{Agradecimentos}

Ao Prof. Dr. Mário Francisco Mucheroni, pela orientação segura e paciente na elaboração deste trabalho. Pelo incentivo, valiosa amizade e compreensão, o meu muito obrigada.

Ao Prof. Titular Eduardo Morgado Belo pela confiança que depositou em mim.

Ao Prof. Dr. Roberto Hideaki Tsunaki pelas valiosas discussões que contribuíram para o desenvolvimento deste trabalho.

Ao Prof. Dr. Benedito Di Giacomo, o Benê, por ter me acolhido no laboratório de metrologia durante todo o tempo que precisei.

Aos meus amigos Cláudio e Edson pela preocupação, e presteza com a qual fui atendida sempre que solicitei, principalmente durante a realização do ensaio experimental.

As minhas amigas Andréa, Érica, Elizangela e Rosenda pelo apoio e pelas palavras de incentivo. A Cecília, muito obrigada.

À minha amiga Dani, com quem pude contar sempre.... obrigada por tudo.

À minha amiga Jú, quem sempre me apoiou, me ouviu e me deu muitos conselhos desde o tempo da graduação.

Aos colegas e funcionários do laboratório de metrologia pela consideração e amizade.

Aos meus pais Jesus e Maria por me ensinar desde muito cedo, a simplicidade e a magnitude da vida. Por terem me ouvido, me incentivado e me dado tanto apoio.....amo muito vocês.

À toda minha família.

Ao meu marido Francisco, pelo amor, compreensão e muita paciência e por todo seu apoio, minha eterna gratidão.

À minha filhota Ana Laura, que Deus me concedeu, o melhor presente de toda minha vida.

À CAPES, pela bolsa de estudo concedida.

E acima de tudo à DEUS, por permitir que a vida aconteça. 


\section{Resumo}

Neves, A. T. (2006). Aplicação dos Modelos Paramétricos ARMAV e ARV na

Identificação Modal de Sistemas Mecânicos. Tese (Doutorado) - Escola de

Engenharia de São Carlos, Universidade de São Paulo, São Carlos, 2006.

A análise modal experimental tem contribuído de forma decisiva para caracterização e solução de problemas de engenharia, relacionados à vibração estrutural. Uma das áreas fundamentais da análise modal experimental é a identificação de sistemas, cujo objetivo é determinar as propriedades dinâmicas de uma estrutura, descritas através das freqüências naturais, fatores de amortecimento e modos de vibrar do sistema em análise. Neste trabalho é realizado um estudo sobre as técnicas paramétricas de identificação de sistemas no domínio do tempo utilizando o modelo Auto-Regressivo de Média Móvel Vetorial (ARMAV) e o modelo Auto-Regressivo Vetorial (ARV). Em ambos os modelos, os procedimentos de identificação dos parâmetros auto-regressivos, responsáveis pela dinâmica do sistema, são estimados utilizando a aproximação dos mínimos quadrados. A partir desses coeficientes um modelo em espaço de estado do sistema é construído, a fim de estimar os parâmetros modais do sistema dinâmico. A ordem do modelo ARMAV, necessária para determinar as características dinâmicas do sistema, é estimada através do critério de informação Bayesiana (BIC). Para o caso do procedimento baseado no modelo $\mathrm{ARV}$, onde apenas as respostas do sistema são consideradas no processo de identificação, uma nova técnica é proposta para solucionar o problema da identificação da ordem do modelo dinâmico. Essa técnica, baseada na estabilidade das freqüências naturais estimadas em várias identificações, contribuiu também para automação do procedimento de identificação. $O$ desempenho dos algoritmos de identificação utilizando o modelo ARMAV, e o modelo ARV juntamente com a nova metodologia desenvolvida, é verificado através de aplicações a dados provenientes de simulações numéricas e de um ensaio experimental realizado em uma placa de alumínio.

Palavras-chave: modelos ARMAV e ARV; identificação de sistemas; método dos mínimos quadrados, ordem do modelo. 


\section{Abstract}

\section{Neves, A. T. (2006). Application of ARMAV and ARV Parametric Models in the}

Modal Identification of Mechanical Systems. Ph. D. Thesis - Escola de Engenharia de

São Carlos, Universidade de São Paulo, São Carlos, 2006.

The experimental modal analysis has contribued in a decisive way to characterization and solution of engineering problems, related to structural vibration. One of the fundamental areas of the experimental modal analysis is the mechanical systems identification, whose objective is to identify the dynamic properties of a structure, described through the natural frequencies, damping ratios and mode shapes of the system in analysis. In this work a study is accomplished on the parametric techniques of systems identification in time domain using the Auto-Regressive Moving Average Vector (ARMAV) and the Auto-Regressive Vector (ARV) models. In these models, the procedures of the auto-regressive parameters identification that describes the dynamics of the system are estimated using the least square approach. Trough these coefficients a model in state space is built, in order to identify the modal parameters of the dynamic system. The order of the ARMAV model, necessary to determine the dynamic characteristics of the system, is estimated through Bayesian Information Criterion (BIC). For the procedure based on the model ARV, where only the system responses are considered in the identification process, a new technique is proposed to solve the identification problem of the order of the dynamic model. This technique, based on the stability of the natural frequencies in several identifications, also contributed to automation of the identification procedure. The performance of these identification algorithms using the ARMAV model, and the ARV model together with the new developed methodology, is verified using data from numerical simulations and from an experimental test accomplished in an aluminum plate.

Keywords: ARMAV and ARV models; systems identification; least-square method, model order. 


\section{Lista de Figuras}

1.1 Fases da análise modal teórica (a) e experimental (b) ................................. 18

6.1 Fluxograma do procedimento de identificação - modelo ARMAV ...................83

6.2 Fluxograma do procedimento de identificação - modelo ARV .........................84

6.3 Fluxograma da seleção das melhores estimativas....................................87

6.4 Sistema massa - mola - amortecedor de $n$ gdl..................................... 89

6.5 Diagramas de estabilidade - modelo ARMAV - (caso 1): (a) ruído 0\% ( $L=500)$;

(b) ruído $0 \%\left(L=L_{\max }\right)$; (c) ruído 5\% $(L=500)$; (d) ruído 5\% $\left(L=L_{\max }\right) \ldots \ldots \ldots . . .93$

6.6 Critério BIC aplicado ao modelo ARMAV - (caso 1).................................93

6.7 Comparação entre as formas dos modos de vibrar teóricos e identificados modelo ARMAV - (caso 1$)$..........................................................96

6.8 Teste do sinal residual da saída 1 (a) função de auto-correlação; (b) histograma (caso 1$)$

6.9 Diagramas de estabilidade - modelo ARV - (caso 1) (a) ruído 0\%; (b) ruído5\%.100

6.10 Comparação entre as formas dos modos de vibrar teóricos e identificados modelo ARV - (caso 1$)$

6.11 Diagramas de estabilidade - modelo ARMAV- (caso 2): (a) ruído 0\% ( $L=500$ );

(b) ruído $\quad 0 \% \quad\left(L=L_{\max }\right)$; $\quad$ (c) ruído $5 \% \quad(L=500)$; (d) ruído $5 \%$

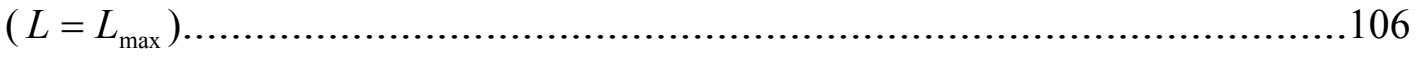

6.12 Critério BIC aplicado ao modelo ARMAV- (caso 2)................................106

6.13 Comparação entre as formas dos modos de vibrar teóricos e identificados - modelo

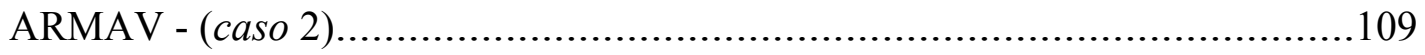

6.14 Teste do sinal residual da saída 1 (a) função de auto-correlação; (b) histograma (caso 2).

6.15 Diagramas de estabilidade - modelo ARV- (caso 2) (a) ruído 0\%; (b) ruído $5 \%$.

6.16 Comparação entre as formas dos modos de vibrar teóricos e identificados - modelo ARV - (caso 2).

7.1 Placa de alumínio utilizada no ensaio experimental. 
7.2 Descrição da placa de alumínio e localização dos pontos de entrada e saída.......120

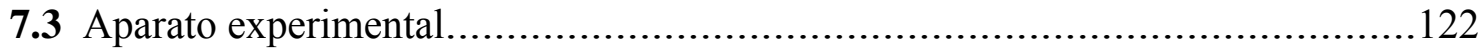

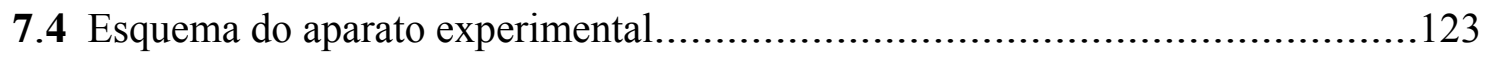

7.5 Espectro de potência médio - ponto de excitação $\boldsymbol{A}$ e $\boldsymbol{B}$.............................124

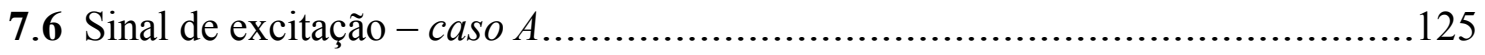

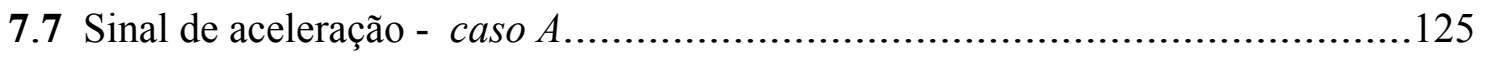

7.8 Diagramas de estabilidade - modelo ARMAV- (a) caso A; (b) caso B................126

7.9 Critério BIC aplicado ao modelo ARMAV- (caso A)..................................127

7.10 Teste do sinal residual da saída 2 (a) função de auto-correlação; (b) histograma - (

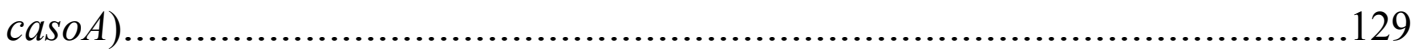

7.11 Diagramas de estabilidade - modelo ARV- (a) caso A; (b) caso B..................130

7.12 Auto-espectro de potência médio de saída - ponto de excitação $\boldsymbol{A}$ e $\boldsymbol{B}$..............131

7.13 Distribuição dos pontos de excitação e resposta sobre a placa utilizada na representação gráfica dos modos.................................................133

$7.141^{\mathrm{o}}$ modo de vibrar da placa de alumínio......................................... 134

$7.152^{\circ}$ modo de vibrar da placa de alumínio..........................................135

$7.163^{\mathrm{o}}$ modo de vibrar da placa de alumínio........................................135

$7.174^{\mathrm{o}}$ modo de vibrar da placa de alumínio...........................................136

$7.185^{\circ}$ modo de vibrar da placa de alumínio...........................................136 


\section{Lista de Tabelas}

6.1 Parâmetros físicos e parâmetros modais teóricos - modelo $4 \mathrm{gdl} . . . . . . . . . . . . . . . . . . . .91$

6.2 Modos de vibrar teóricos - modelo 4gdl............................................92

6.3 Parâmetros modais identificados - modelo ARMAV - (caso 1)........................94

6.4 Modos de vibrar identificados - modelo ARMAV - (caso 1).........................95

6.5 Erros dos modos identificados - modelo ARMAV - (caso1) ..........................95

6.6 Valores assumidos por MAC - modelo ARMAV - (caso 1) ...........................97

6.7 Erros dos parâmetros modais identificados considerando número reduzido de pontos de medição - modelo ARMAV- (caso 1) ..............................................98

6.8 Valores assumidos por MAC - modelo ARMAV - (caso 1) considerando número reduzido de pontos de medição ..................................................98

6.9 Parâmetros modais identificados - modelo ARV- (caso 1)............................101

6.10 Modos de vibrar identificados - modelo ARV - (caso 1)...........................101

6.11 Erros dos modos identificados - modelo ARV - (caso1)............................102

6.12 Valores assumidos por MAC - modelo ARV - (caso 1) …..........................102

6.13 Erros dos parâmetros modais identificados considerando número reduzido de pontos de medição - Modelo ARV- (caso 1)...........................................103

6.14 Valores assumidos por MAC - modelo ARV - (caso 1) considerando número reduzido de pontos de medição .......................................................104

6.15 Parâmetros físicos e parâmetros modais teóricos - modelo $5 \mathrm{gdl}$....................105

6.16 Modos de vibrar teóricos - modelo 5gdl.......................................... 105

6.17 Parâmetros modais identificados - modelo ARMAV - (caso 2).....................107

6.18 Modos de vibrar identificados - modelo ARMAV - (caso 2).......................108

6.19 Erros dos modos identificados - modelo ARMAV - (caso 2).......................108

6.20 Valores assumidos por MAC - modelo ARMAV - (caso 2) .........................109

6.21 Erros dos parâmetros modais identificados considerando número reduzido de pontos de medição - modelo ARMAV - (caso 2). 
6.22 Valores assumidos por MAC - modelo ARMAV - (caso 2) considerando número reduzido de pontos de medição

6.23 Parâmetros modais identificados - modelo ARV - (caso 2).......................113

6.24 Modos de vibrar identificados - modelo ARV - (caso 2)............................114

6.25 Erros dos modos identificados - modelo ARV - (caso 2)...........................114

6.26 Valores assumidos por MAC - modelo ARV - (caso 2) …........................115

6.27 Erros dos parâmetros modais identificados considerando número reduzido de pontos de medição - modelo ARV - (caso 2)...........................................116

6.28 Valores assumidos por MAC - modelo ARV - (caso 2) considerando número reduzido de pontos de medição

7.1 Equipamentos utilizados no experimento........................................ 122

7.2 Parâmetros modais identificados - modelo ARMAV - (caso A e caso B)..........127

7.3 Estimativas das freqüências obtidas por diferentes modelos.......................128

7.4 Máximo erro relativo percentual - freqüências (ARMAV) .......................... 128

7.5 Parâmetros modais identificados - modelo ARV - (caso A e caso B)...............130

7.6 Estimativas das freqüências naturais obtidas pelos $A E P M \ldots \ldots \ldots \ldots \ldots \ldots \ldots \ldots \ldots \ldots 131$

7.7 Máximo erro relativo percentual - freqüências (ARV) .............................132

7.8 Média e desvios-padrão das freqüências.................................................134 


\section{Lista de Simbolos}

$J(\theta)$ função critério escalar;

$m \quad$ número de saídas;

$n \quad$ graus de liberdade do sistema de segunda ordem;

$p \quad$ ordem das matrizes polinomiais auto-regressiva $A(q)$ e média móvel $B(q)$

$r \quad$ número de entradas;

$t \quad$ variável temporal;

$\alpha_{j} \quad j$-ésimo autovalor de $A_{c}$;

$\delta(j)$ delta de Kronecker;

$\Delta t \quad$ intervalo de tempo constante;

$\zeta_{j} \quad j$-ésimo fator de amortecimento;

$\lambda_{j} \quad j$-ésimo autovalor de $A_{d}$

$\omega_{d j} \quad j$ - ésima freqüência amortecida;

$\omega_{j} \quad j$ - ésima freqüência natural;

$f(t) \quad$ vetor força nas coordenadas generalizadas; $(n \times 1)$

$s(k) \quad$ ruído do processo; $(r \times 1)$

$u(t) \quad$ vetor entrada; $(r \times 1)$

$u(k) \quad$ vetor entrada do sistema em tempo discreto; $(r \times 1)$

$v(k) \quad$ ruído de medida; $(m \times 1)$

$x(t) \quad$ vetor de estado; $(2 n \times 1)$

$x(k) \quad$ vetor de estado em tempo discreto; $(2 n \times 1)$

$y(t) \quad$ vetor saída; $(m \times 1)$

$y(k) \quad$ vetor saída do sistema em tempo discreto; $(m \times 1)$

$\hat{y} \quad$ vetor preditor de resposta; $(m \times 1)$

$w(t) \quad$ vetor de deslocamento generalizado; $(n \times 1)$

$\dot{w}(t) \quad$ vetor de velocidade generalizado; $(n \times 1)$

$\ddot{w}(t) \quad$ vetor de aceleração generalizado; $(n \times 1)$

$\varepsilon(k) \quad$ vetor erro residual; $(m \times 1)$

$\phi_{j} \quad j$-ésimo modo de vibrar; $(m \times 1)$ 
$A_{c} \quad$ matriz de estado em tempo contínuo; $(2 n \times 2 n)$

$A_{d} \quad$ matriz de estado em tempo discreto; $(2 n \times 2 n)$

$A_{i} \quad i$-ésimo coeficiente matricial auto-regressivo; $(m \times m)$

$A(q)$ matriz polinomial auto-regressiva; $(m \times m)$

$B_{c}$ matriz de influência de entrada em tempo contínuo; $(2 n \times r)$

$B_{d} \quad$ matriz de influência de entrada em tempo discreto; $(2 n \times r)$

$B_{i} \quad i$-ésimo coeficiente matricial média móvel; $(m \times r)$

$B(q)$ matriz polinomial média móvel; $(m \times r)$

C matriz de amortecimento; $(n \times n)$

$C_{c}$ matriz de influência de saída em tempo contínuo; $(m \times 2 n)$

$C_{d} \quad$ matriz de influência de saída em tempo discreto; $(m \times 2 n)$

$D_{c} \quad$ matriz de transmissão direta em tempo contínuo; $(m \times r)$

$D_{d} \quad$ matriz de transmissão direta; $(m \times r)$

$\hat{E} \quad$ matriz composta dos erros residuais; $(m \times L)$

$F \quad$ matriz de influência de entrada; $(n \times r)$

$h(j)$ função de resposta ao impulso; $(m \times r)$

$H(q)$ função transferência; $(m \times r)$

$K \quad$ matriz de rigidez; $(n \times n)$

$L_{d} \quad$ matriz de influência de saída para o deslocamento; $(m \times n)$

$L_{v} \quad$ matriz de influência de saída para a velocidade; $(m \times n)$

$L_{a} \quad$ matriz de influência de saída para a aceleração; $(m \times n)$

$M \quad$ matriz de massa; $(n \times n)$

$Q_{o}(v)$ matriz de observabilidade; $(m v \times 2 n)$

$Q_{c}(v)$ matriz de controlabilidade; $(2 n \times v r)$

$R(j)$ função correlação dos dados de saída; $(m \times m)$

$\hat{S} \quad$ matriz composta dos estimadores de resposta e entrada; $((m p+r p+r) \times L)$

$\widetilde{U}$ e $\widetilde{S}$ matrizes compostas por matrizes de função de correlação de saídas; $(m \widetilde{L} \times m)$ e $(m \tilde{L} \times m p)$, respectivamente

$\hat{Y} \quad$ matriz composta dos estimadores de resposta; $(m \times L)$

$\Delta \quad$ matriz covariância do processo ruído branco $u(k) ;(r \times r)$ 
$\hat{\theta} \quad$ estimativa dos parâmetros auto-regressivo e de média móvel; $(m \times(m p+r p+r))$

$\widetilde{\theta}$ estimativa dos parâmetros auto-regressivo; $(\mathrm{mp} \times m)$

$\Phi \quad$ matriz modal; $(m \times 2 n)$

$\Omega \quad$ matriz covariância do erro residual; $(m \times m)$

$E[$.$] \quad operador esperança;$

$q^{-i} \quad$ operador de atraso;

$\operatorname{tr}($ ) operador traço;

o operador derivada; 


\title{
Lista de Abreviaturas
}

\author{
AIC Critério de Informação de Akaike \\ AMO Análise Modal Operacional \\ AMT Análise Modal Tradicional \\ ARMA Modelo Auto-Regressivo de Média Móvel \\ ARMAV Modelo Auto-Regressivo de Média Móvel Vetorial \\ ARMAX Modelo Auto-Regressivo de Média Móvel com entrada Exógena \\ ARV Modelo Auto-Rregressivo Vetorial \\ AEPM Auto-Espectro de Potência Médio \\ BIC Critério de Informação Bayesiana \\ CE Método da Exponencial Complexa \\ ERA Algoritmo de Realização de Sistemas \\ EPM Espectro de Potência Médio \\ FRF Função de Resposta em Freqüência \\ FPE Critério do Erro Preditor Final \\ IRF Função de Resposta ao Impulso \\ ITD Método de Ibrahim no Domínio do Tempo \\ IV Método da Variável Instrumental \\ LSCE Método dos Mínimos Quadrados para Exponenciais Complexas \\ LMS Método dos Mínimos Quadrados em Múltiplos Estágios \\ LS Método dos Mínimos Quadrados \\ MAC Critério de Correlação Modal \\ MDL Critério de Comprimento de Descrição Mínima \\ MIMO Múltiplas Entradas e Múltiplas Saídas \\ ML Método da Máxima Verossimilhança \\ PEM Método do Erro Preditor \\ PRCE Método de Referência Múltipla \\ RIV Método da Variável Instrumental Recursivo \\ RLS Método dos Mínimos Quadrados Recursivo \\ SISO Única Entrada e Única Saída \\ SSI Método Estocástico de Subespaço \\ SVD Decomposição em Valores Singulares
}




\section{Sumário}

\section{Introdução}

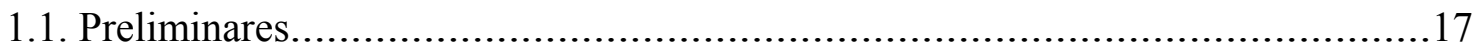

1.2. Análise Modal Tradicional e Análise Modal Operacional .............................19

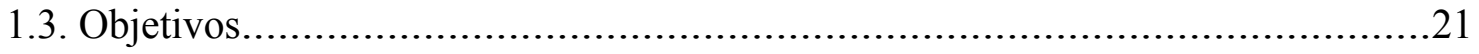

1.4. Organização da Tese....................................................................21

\section{Revisão Bibliográfica}

2.1. Preliminares

2.2.Considerações Gerais sobre os Métodos de Identificação

2.3. Métodos Clássicos de Identificação no Domínio do Tempo. .25

2.4. Revisão de Literatura - Modelo ARMA e suas Variações.

\section{Representação de Sistemas Dinâmicos em Espaço de Estado}

3.1. Preliminares 35

3.2. Modelagem em Espaço de Estado - Sistema em Tempo Contínuo.....................36

3.3. Modelagem em Espaço de Estado - Sistema em Tempo Discreto......................39

3.4. Modelo de Estado nas Coordenadas Modais. .42

4. Desenvolvimento Teórico da Relação de Equivalência entre o Modelo de Espaço de Estado e o Modelo ARMAV

4.1. Preliminares. 
4.2. Modelagem de Sistemas Determinísticos e Estocásticos.

4.3. Descrição do Modelo ARMAV ...........................................................50

4.4. Relação entre o Modelo ARMAV e o Modelo de Espaço de Estado...................51

4.5. Uma Realização em Espaço de Estado do Modelo ARMAV............................56

4.5.1. Função de Resposta ao Impulso do Modelo ARMAV ............................56

4.5.2. A Realização em Espaço de Estado - Observabilidade Canônica...............58

4.6. Decomposição Modal da realização em Espaço de Estado do Modelo ARMAV...64

\section{Identificação de Sistemas Utilizando o Modelo ARMAV e ARV}

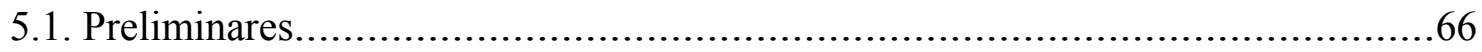

5.2. Métodos de Estimação dos Parâmetros dos Modelos ARMAV e ARV ...............67

5.2.1. Método dos Mínimos Quadrados - Modelo ARMAV ..........................67

5.2.2. Método dos Mínimos Quadrados - Modelo ARV ...............................70

5.2.3. Método dos Mínimos Quadrados Modificado - Modelo ARV................74

5.3. Procedimentos de Determinação da Ordem do Modelo...............................77

5.4. Validação do Modelo.................................................................. 80

\section{Aspectos Computacionais e Simulações Numéricas}

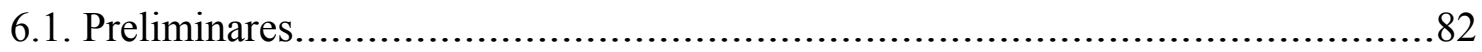

6.2. Procedimentos de Identificação...................................................... 83

6.3. Simulações Numéricas de Sistemas Dinâmicos.........................................89

6.3.1. Sistema Dinâmico de 4 GDL $-1^{\circ}$ CASO..........................................91

6.3.2. Sistema Dinâmico de 5 GDL $-2^{\circ}$ CASO.....................................104 


\section{Aplicação Experimental}

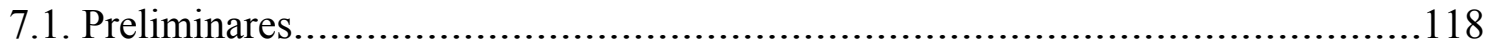

7.2. Descrição do Modelo Experimental..........................................................118

7.3. Aquisição e Processamento dos Sinais................................................120

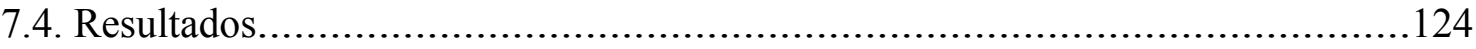

\section{Conclusões e Sugestões}

8.1. Conclusões Gerais 138

8.2. Proposta para Futuros Desenvolvimentos. 140

Referências Bibliográficas 141

Apêndice A - Função de Correlação 


\section{Introdução}

\subsection{PRELIMINARES}

As grandes descobertas científicas, juntamente com um acelerado desenvolvimento tecnológico e uma economia cada vez mais globalizada em busca de mercados competitivos vem impondo novos desafios à sociedade. O caráter global das relações comerciais, a competitividade e a luta por maiores parcelas do mercado, levaram as indústrias e universidades a investirem na procura de novas tecnologias com o objetivo de alcançar maior qualidade dos produtos, de forma a satisfazer a exigência dos centros consumidores.

A fabricação de produtos de qualidade envolve uma série de determinações e normalizações que especificam condições de operação ou utilização destes produtos. No contexto da engenharia mecânica, uma das características que afetam diretamente a qualidade destes produtos é o seu comportamento dinâmico. Desta forma, torna-se necessário o conhecimento de técnicas que possibilitem encontrar as características dinâmicas das estruturas mecânicas. Tais características podem ser determinadas através de dois procedimentos (EWINS,1984).

O primeiro denominado Análise Modal Teórica pertence à classe dos chamados problemas diretos. As fases deste procedimento são mostradas na figura 1.1 (a). Este 
procedimento consiste inicialmente na formulação de um modelo matemático descrito através das matrizes de massa e rigidez de estruturas obtidas, por exemplo, utilizando o método de elementos finitos. A essa descrição dá-se o nome de modelo espacial. Em seguida, essas matrizes são utilizadas na formulação de um problema de autovalor cuja solução fornece as características dinâmicas da estrutura, ou seja, os modos normais de vibração e suas respectivas freqüências naturais, denominado modelo modal.

É importante ressaltar que esta solução descreve as várias formas nas quais a estrutura é capaz de vibrar naturalmente com certas condições iniciais, sem qualquer força externa ou excitação e, portanto, tais modos são chamados de modos normais ou naturais de vibrar da estrutura. A terceira fase determina como e com quais amplitudes a estrutura irá vibrar sob certas condições de excitação. Naturalmente, a resposta do sistema não dependerá somente das propriedades inerentes à estrutura, mas também da origem e magnitude da excitação imposta. A resposta da estrutura a uma forma de excitação padronizada é dita modelo de resposta, que consiste em um conjunto de funções de respostas em freqüência (FRF), as quais devem ser definidas em uma faixa de freqüência de interesse.

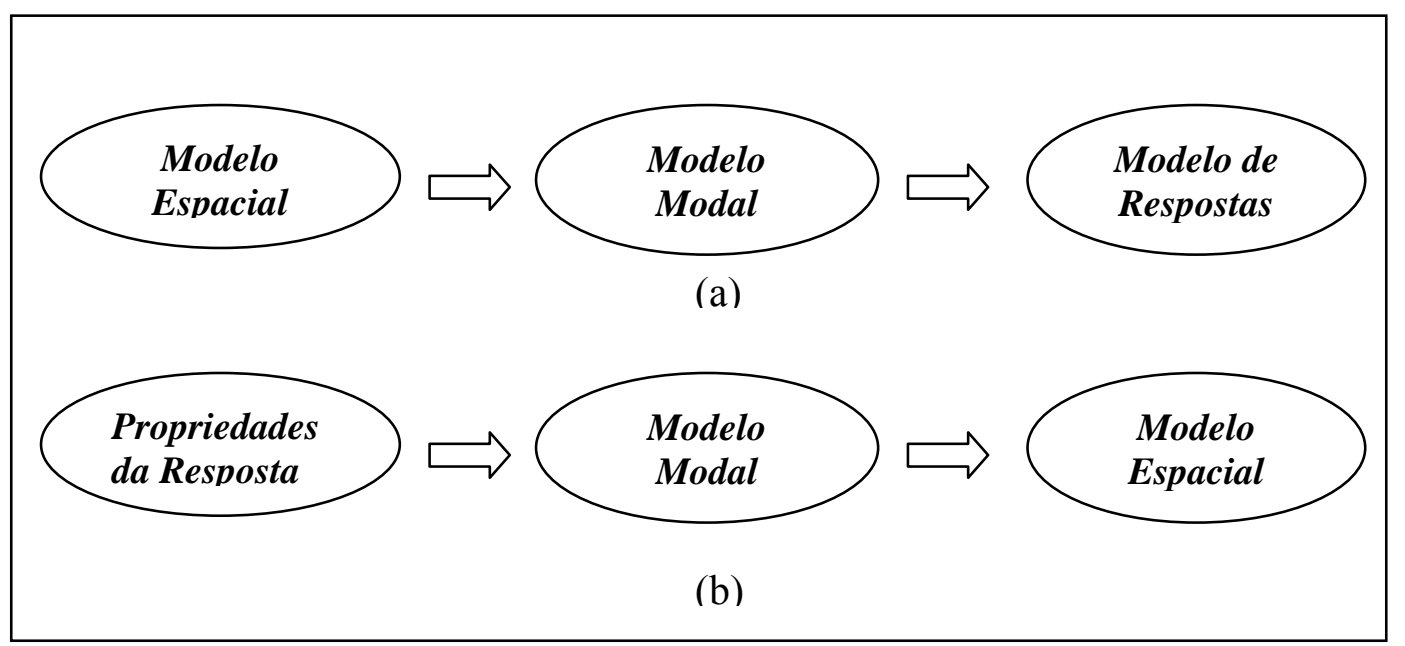

Figura 1.1. Fases da análise modal teórica (a) e experimental (b)

O segundo procedimento chamado Análise Modal Experimental é classificado como problema inverso, cujas fases podem ser vistas na figura 1.1 (b). Essa técnica inicia-se através da utilização das propriedades das respostas obtidas experimentalmente, provenientes de medições sobre a estrutura, para obter as propriedades dinâmicas e a partir destes dados extrair as características espaciais. Geralmente, esse procedimento corresponde a um ajuste de curvas aos dados experimentais e posterior determinação dos parâmetros modais. 
Como desvantagens na formulação do modelo matemático, imprescindíveis no procedimento da análise modal teórica estão: a necessidade do estabelecimento de hipóteses simplificadoras, a falta de conhecimento das condições de contorno físicas do sistema real e o quase total desconhecimento da matriz de amortecimento, que representa a dissipação de energia vibratória pelo sistema. Essas limitações causadas no processo da análise dinâmica acabam produzindo resultados imprecisos.

Tendo em vista essas limitações, geralmente faz-se necessário a análise experimental na obtenção do modelo modal, quer seja para comparar ou corrigir o modelo obtido pela análise modal teórica, ou até mesmo nos casos que torna-se impossível obter um modelo analítico através da análise modal teórica.

Segundo Maia e Silva (1997), a análise modal experimental foi aplicada pela primeira vez com sucesso por volta de 1940, na análise do comportamento dinâmico de uma estrutura aeronáutica.

Nos últimos anos, com o desenvolvimento e difusão de equipamentos apropriados, esta técnica tem sido aplicada não somente nas indústrias: aeroespacial, automotiva e de máquinas de precisão, mas também expandida para outros setores, como por exemplo: eletrodomésticos e eletro-eletrônicos. Essa expansão é plenamente justificável, uma vez que esses produtos agregam conteúdo tecnológico devendo ser submetidos às normas de produção e a um alto controle de qualidade para que possam ser mantidos no mercado competitivo.

A análise modal experimental também possui uma estreita relação com temas de pesquisa em análise vibroacústica de sistemas mecânicos além de ser largamente empregada em estruturas de engenharia civil.

\subsection{ANÁliSE MODAL TRADICIONAL E ANÁlISE MODAL OPERACIONAL}

A análise modal experimental pode estar relacionada à Análise Modal Tradicional (AMT) ou à Análise Modal Operacional (AMO), também conhecida como Análise Modal Ambiente, de Resposta ou ainda de Entrada Natural.

No caso da AMT, o modelo modal é identificado de forma tradicional, a partir dos dados de entrada e saída do sistema, enquanto que na $\mathrm{AMO}$, o modelo é obtido 
utilizando-se apenas as respostas de vibração, uma vez que as fontes de excitação são consideras conhecidas somente no sentido estatístico, e em geral, aproximada por um processo de ruído branco (DESFORGES, COOPER e WRIGHT, 1995).

A AMO é utilizada com freqüência em estruturas mecânicas de grande porte; estruturas muito flexíveis ou estruturas em condição de operação que não são fáceis de serem excitadas artificialmente, como por exemplo, eixos rotativos apoiados em rolamentos, plataformas marítimas, pontes, edifícios, dentre outros.

Estas estruturas, além de não serem facilmente excitadas, geralmente possuem forças de excitação natural (ambiente) que não podem ser controladas ou medidas. Dentre os vários exemplos de excitação natural, encontram-se: o carregamento de ondas em plataformas marítimas, forças causadas pelo tráfego em pontes e excitações provocadas pelo vento em edifícios.

A aplicação da AMO traz alguns benefícios em relação a AMT, dentre os quais pode-se mencionar: a facilidade e a redução de custos na realização de ensaios modais em laboratórios, uma vez que não há necessidade de equipamentos para medir forças de excitação; a não necessidade da interrupção das condições de operação da estrutura durante a medição, e da utilização de dispositivos de vibração forçada em situações reais, evitando assim transtornos e gastos financeiros excessivos.

Tendo em vista a importância e o crescente campo de aplicação da análise modal experimental, o presente trabalho está relacionado com o estudo de técnicas de identificação do modelo modal, em particular no domínio do tempo, aplicado à análise de sistemas mecânicos.

O modelo paramétrico vetorial auto-regressivo de média móvel - ARMAV (do inglês, Auto-Regressive Moving Average Vector) e o modelo constituído somente da parcela auto-regressiva - ARV (do inglês, Auto-Regressive Vector), cujas fontes de excitação são não observáveis, serão utilizados como base das técnicas de identificação aqui estudadas.

A escolha desses modelos, formulados na teoria de séries temporais, deve-se ao fato dos mesmos aparecerem na literatura de forma bem consolidada na teoria de identificação modal de sistemas.

Uma revisão literária e as considerações gerais sobre os principais métodos de identificação, sobretudo de técnicas provenientes do modelo da classe ARMA, serão apresentadas no capítulo dois. 


\subsection{OBJETIVOS}

Esta tese tem como objetivo realizar um estudo sobre técnicas paramétricas de identificação de sistemas no domínio do tempo utilizando o modelo ARMAV e ARV.

A partir desse estudo, implementar algoritmos computacionais que descrevem procedimentos de identificação com base nos modelos ARMAV e ARV, utilizando respectivamente o método dos mínimos quadrados e o método dos mínimos quadrados modificado.

Essa modificação é fundamentada na equivalência entre a resposta livre de um sistema linear e a função de correlação dos dados de respostas de um sistema, excitado por um ruído branco gaussiano de média zero.

Para o caso do procedimento baseado no modelo ARV, propor uma nova metodologia no processo de identificação estrutural que contribui para automatizar o procedimento, bem como para encontrar a ordem que melhor representa o sistema, dentro da faixa de freqüência de interesse. Esta técnica, baseada na estabilidade dos modos estruturais aliada à aplicação do método da regressão linear entre as freqüências estimadas em várias identificações, elimina os modos computacionais sem a necessidade da utilização de índices de confiança, e consequentemente encontra uma estimativa final para os parâmetros modais do sistema.

Como parte também dos objetivos traçados, o desempenho dos algoritmos de identificação é verificado através de suas aplicações aos dados provenientes de simulações numéricas e de um ensaio experimental. Os testes simulados são realizados a partir da análise de sistemas com características semelhantes aos sistemas físicos reais, cujos sinais de saída foram corrompidos por ruído e um número reduzido de medidas de respostas consideradas. Já o ensaio experimental é realizado numa placa de alumínio engastada em um dos lados.

\subsection{ORGANIZAÇÃO DA TESE}

O trabalho foi organizado de forma a apresentar uma seqüência de tópicos necessários para o seu desenvolvimento. 
Deste modo, a tese foi dividida em oito capítulos descritos a seguir.

Este primeiro capítulo apresenta uma introdução ao assunto abordado nesta tese; os objetivos deste trabalho e como a tese está organizada.

No capítulo dois é realizada uma revisão bibliográfica, onde são apresentados os trabalhos mais importantes nessa área de pesquisa.

No capítulo três é descrita a representação de um sistema dinâmico na modelagem de espaço de estado em tempo contínuo e tempo discreto.

O capítulo quatro descreve o desenvolvimento teórico da relação existente entre o modelo ARMAV e o modelo de espaço de estado.

No capítulo cinco é realizada a descrição dos métodos para estimar os coeficientes dos modelos ARMAV e ARV através da aproximação dos mínimos quadrados, que serão utilizados no cálculo da estimativa dos parâmetros modais. Além disso, este capítulo ainda trata dos critérios para determinação da ordem do modelo, bem como para sua validação.

O capítulo seis apresenta os algoritmos computacionais utilizados no processo de identificação bem como as aplicações numéricas, resultados e discussões.

O capítulo sete traz a aplicação dos algoritmos de identificação apresentados a dados provenientes de um ensaio experimental realizado em uma placa de alumínio.

No capítulo oito são apresentadas as conclusões gerais, com base nos resultados alcançados e sugestões para futuros desenvolvimentos.

Finalmente, no Apêndice A são apresentadas a definição e algumas propriedades da função de correlação utilizadas no capítulo cinco. 


\section{Revisão Bibliográfica}

\subsection{PRELIMINARES}

Dentro da Teoria de Identificação de Sistemas, uma das áreas fundamentais, a análise modal experimental, tem contribuído de forma grandiosa na identificação e na solução de problemas de engenharia, relacionados à vibração estrutural.

O fenômeno de vibração estrutural é intrínseco aos sistemas e depende da distribuição espacial da massa, amortecimento e rigidez da estrutura.

No universo mecânico existem inúmeras situações nas quais a análise desse fenômeno é indispensável ao projeto, sob pena de inviabilizá-lo ou até mesmo provocar a destruição da estrutura.

Como exemplo pode-se mencionar os problemas de ruído em motores e máquinas girantes, desgaste exagerado por fadiga estrutural, o conforto acústico de ambientes, o fenômeno de flutter que ocorre na asa de aeronaves, como uma infinidade de outras aplicações de análise de vibração estrutural.

Nas últimas décadas um variado conjunto de técnicas de identificação de sistemas tem sido desenvolvido e aplicado ao estudo dos fenômenos vibratórios em estruturas mecânicas. Essas técnicas buscam determinar as propriedades dinâmicas da estrutura, descritas através de um conjunto de parâmetros, denominados parâmetros 
modais, que caracterizam as freqüências naturais, fatores de amortecimento e modos de vibrar do sistema em análise, a partir de dados experimentais.

Esses parâmetros identificados constituem informações que podem ser utilizadas como ferramenta, sobretudo para corrigir ou validar modelos analíticos, detectar a presença de falhas estruturais, realizar modificação estrutural, se necessário; e realizar o controle de qualidade e monitoramento da vida útil da estrutura em estudo.

Neste contexto, o presente capítulo apresenta uma descrição geral sobre as técnicas de identificação e uma revisão bibliográfica dos métodos que utilizam a classe do modelo ARMA no processo de estimação dos parâmetros modais de uma estrutura vibrante.

\subsection{CONSIDERAÇÕES GERAIS SOBRE OS MÉTODOS DE IDENTIFICAÇÃO DE SISTEMAS}

Especialmente a partir de 1970, graças aos avanços tecnológicos, muitos trabalhos relacionados à Teoria de Identificação de Sistema foram desenvolvidos. Com isso, novos métodos e técnicas de identificação de parâmetros modais surgiram resultantes das facilidades proporcionadas pela implementação de um algoritmo que realizasse de maneira eficiente o cálculo computacional da série de Fourier (COOLEY e TUKEY, 1965).

Outro fator de grande relevância, responsável por esse desenvolvimento, é a evolução do aparato experimental, como a utilização de analisadores espectrais, computadores e equipamentos de modo geral, que permitem a aquisição e tratamento de grande quantidade de dados.

Os métodos de identificação podem se diferenciar entre si, no que se refere ao tipo e a quantidade de dados de entrada e saída e quanto ao tipo de processamento matemático dos dados.

Quando os testes modais são realizados, considerando a técnica do tipo entrada única e saída única (SISO), as medições dos sinais de excitação e resposta devem ser coletadas mudando a localização da excitação quantas vezes for necessário, a fim de poder completar a informação modal requerida. O fato de mudar as localizações da excitação e de colher os sinais dinâmicos em tempos diferentes pode acarretar em variações das características dinâmicas do sistema se o sistema estiver mudando as 
condições de operação, isto contradiz a hipótese de se trabalhar com modelos invariantes no tempo, o que é uma desvantagem da metodologia SISO (BAZÁN, 1993).

Além disso, as técnicas SISO aplicadas a sistemas com modos fortemente acoplados e/ou alto grau de amortecimento fornecem resultados pouco precisos (EWINS, 1984). Esta imprecisão nos resultados também é verificada nos casos de sistemas que apresentam amortecimento extremamente pequeno.

As técnicas que consideram, uma única excitação e múltiplas saídas (SIMO), e múltiplos sinais de excitação e resposta do sistema (MIMO), coletados simultaneamente, têm apresentado uma tendência crescente de utilização, quando comparadas às técnicas SISO. Isto se deve ao fato de que além de reduzir significativamente a aquisição de dados e o tempo de análise, permite estimar de maneira mais precisa os parâmetros modais (PAPAKOS e FASSOIS, 2003).

Quanto ao processamento dos dados, podem ser numericamente tratados no domínio da freqüência ou no domínio do tempo.

Os métodos no domínio da freqüência são largamente utilizados em problemas de identificação, no entanto apresentam algumas limitações, como por exemplo, as citadas por Gontier, Smail e Gautier (1993), quando trata-se de sistemas que apresentam frequências próximas e alto grau de amortecimento, especialmente se associado a medidas com alto nível de ruído. Além disso, Maia e Silva (1997) afirmam que o fenômeno de "leakage" torna-se crítico quando a quantidade de dados é pequena, aspecto que impossibilita uma boa resolução em freqüência.

Desde algumas décadas, os métodos no domínio do tempo têm sido utilizados na análise de vibrações mecânicas como alternativa promissora para esses problemas. Estes métodos caracterizam-se pelo cálculo direto dos parâmetros modais a partir dos dados de excitação e resposta no tempo não necessitando do uso da transformada inversa de Fourier, que acarreta erros numéricos. Além disso, geralmente utilizam amostras de dados reduzidas em relação aos métodos no domínio da freqüência.

\subsection{MÉTODOS CLÁSSICOS DE IDENTIFICAÇÃO NO DOMÍNIO DO TEMPO}

A notoriedade dos métodos no domínio do tempo na área de identificação de sistemas tem crescido substancialmente, desde sua introdução através do método de 
Ibrahim (ITD) (IBRAHIM e MIKULCIK, 1973). Esta primeira formulação do método requer o conhecimento das repostas de deslocamento, velocidade e aceleração, que assim satisfazem as condições do equacionamento em variáveis de estado de maneira apropriada. Os dados coletados na estrutura são empregados na forma de resposta livre do sistema e utilizados na construção da matriz do sistema. Com a resolução de um auto problema, as freqüências naturais e fatores de amortecimento são determinados a partir dos autovalores, e os modos de vibrar através das informações dos autovetores.

Em 1977, Ibrahim e Mikulcik desenvolveram uma segunda versão do método ITD, na qual apenas a resposta livre de uma só grandeza da estrutura, como por exemplo, a aceleração, fosse utilizada no processo de identificação. A vantagem da nova versão deste método, com relação ao anterior é a utilização de uma única grandeza durante o procedimento, evitando assim a integração e/ou diferenciação numérica dos dados de resposta.

Dentre os vários trabalhos desenvolvidos utilizando o método IDT, pode ser citada a dissertação de PAZIANI (2002) que apresenta uma aplicação desse método no processo de identificação dos parâmetros modais de uma placa quadrada de alumínio, engastada em um lado e livre nos demais.

O método da Exponencial Complexa, que se baseia na formulação de Prony desenvolvida em 1795, consiste numa técnica local de estimação das freqüências naturais e fatores de amortecimento a partir de uma única função de resposta ao impulso. Estes parâmetros são encontrados através das raízes de um polinômio obtidos com um ajuste de uma série de exponenciais complexas a uma série temporal.

Em 1979, foi proposto o método dos Mínimos Quadrados para Exponenciais Complexas (LSCE), uma extensão do método CE para um procedimento global de estimação (BROWN et al, 1979). Esta nova abordagem permite o processamento simultâneo de várias IRFs obtidas através da excitação da estrutura em um único ponto e medidas de respostas em vários pontos da estrutura.

O método de referência múltipla (PRCE), desenvolvido por Vold et al (1982), constitui uma extensão do método LSCE para uma versão MIMO, incluindo informações não somente de várias localizações de saídas, mais também várias referências de pontos de entrada na estrutura. Este método realiza a identificação dos 
parâmetros modais em dois estágios. No primeiro estágio as freqüências naturais amortecidas e fatores de amortecimento são determinados através dos dados de funções de respostas ao impulso (IRFs). Já o segundo estágio, consiste na obtenção dos resíduos correspondentes a cada modo de vibrar do sistema (DEBLAUWE, BROWN e ALLEMANG, 1987).

Um outro método denominado de algoritmo de realização de sistemas (ERA) no domínio do tempo foi desenvolvido por JUANG e PAPPA. Este método consiste de uma técnica MIMO de realização balanceada baseada na decomposição em subespaços ortogonais (JUANG e PAPPA, 1985). O objetivo fundamental do método é obter a realização mínima, ou seja, ajustar um modelo em espaço de estado de ordem mínima que descreva as características dinâmicas do sistema, a partir da resposta ao impulso. Posteriormente, os parâmetros modais são calculados através da resolução de um autoproblema envolvendo a matriz do sistema realizado (MAIA e SILVA, 1997).

Com base no algoritmo ERA, Tsunaki (1999) desenvolveu um procedimento para identificação de modelos dinâmicos no espaço de estado, através de técnicas e uso de ferramentas que automatizam o processo de identificação. No ano de 2002, Sczibor desenvolveu um trabalho, também utilizando o algoritmo ERA, onde foi realizado um ensaio de vibração no solo de uma estrutura aeronáutica para obtenção das funções de resposta em freqüência da estrutura.

Em certas situações é praticamente impossível realizar a análise modal tradicional, ou seja, obtenção das propriedades dinâmicas a partir das forças de excitação e repostas de vibração. Neste contexto, James III et al (1995) trouxeram uma contribuição teórica significativa, mostrando que é possível extrair os parâmetros modais de uma estrutura excitada por forças conhecidas apenas no sentido estatístico. Os autores demonstraram que as funções de correlação se comportam da mesma forma que as funções de resposta ao impulso, podendo assim, serem utilizadas nos algoritmos de identificação dos métodos da análise modal tradicional. Desta forma, os métodos LSCE, PRCE, ITD e o algoritmo ERA podem ser empregados na identificação dos parâmetros modais utilizando apenas medidas de respostas obtidas em condição operacional.

Cardoso (2006) desenvolveu um trabalho baseando-se no algoritmo ERA, formulado através das funções de correlação dos dados de respostas. Este trabalho 
apresenta uma nova forma de realizar a identificação estocástica de estruturas em condições operacionais, utilizando para tanto a transformada wavelet e uma análise estatística.

Além dos métodos clássicos de identificação apresentados até então, uma notável classe de métodos no domínio do tempo baseada na família do modelo paramétrico auto-regressivo de média móvel (ARMA) tem sido utilizada com sucesso na área de vibração estrutural. Métodos de identificação que utilizam o modelo ARMA vetorial (ARMAV) têm mostrado serem muito eficientes, principalmente quando a excitação do sistema não é conhecida no sentido determinístico e freqüentemente tomada estatisticamente como um ruído branco (MAIA e SILVA, 1997).

Uma aproximação unificada, do ponto de vista da teoria dos sistemas, que mostra o relacionamento entre os métodos de identificação ITD, PRCE, o algoritmo ERA e o modelo ARMA, pode ser encontrada no trabalho de (YANG et al, 1994).

No modelo ARMA as propriedades dinâmicas são descritas parametricamente através de uma equação linear de diferenças, cujos coeficientes chamados de autoregressivo (AR) e de média móvel (MA) governam a relação entrada-saída do sistema.

Diversas técnicas têm sido desenvolvidas para determinar os coeficientes do modelo ARMA, tornando assim possível, a extração das freqüências naturais, fatores de amortecimento e modos de vibrar da estrutura em estudo.

Basicamente os coeficientes podem ser obtidos através de critérios baseados no método da Máxima Verossimilhança (ML, Maximum Likelihood), método dos mínimos quadrados (LS, Least-Square) ou método da variável instrumental (IV, Instrumental Variable).

O método da ML é um critério de estimação estocástica, que visa extrair os coeficientes do modelo com base em dados observados através da maximização de uma função densidade de probabilidade (GHANEM e HONORARY, 1995). O objetivo principal do critério é obter uma aproximação estocástica que converge para o modelo no sentido do mínimo erro quadrático. Essa aproximação é obtida através de um processo de otimização estabelecido de modo a maximizar a verossimilhança da amostra observada. Embora estaticamente este critério apresente boas propriedades assintóticas, seu processamento requer grande quantidade de dados para a obtenção de um estimador com variância pequena. A implementação computacional desse estimador 
é complexa devido a um problema de maximização não-linear (BRAUN, 1986), além de apresentar possíveis erros de convergência.

Os estimadores LS são considerados subconjuntos dos estimadores ML. No entanto, os estimadores LS podem ser aplicados sem que nenhuma descrição probabilística do problema de estimação seja conhecida. Para um processo estacionário com distribuição gaussiana os estimadores ML e LS fornecem o mesmo resultado.

O método da variável instrumental é baseado numa aproximação dos mínimos quadrados modificado. Este método consiste na utilização de variáveis denominadas instrumentais, que devem ser escolhidas de maneira que elas sejam não correlacionadas com os ruídos e altamente correlacionadas com o vetor de dados amostrados do sinal. A eficiência desse método depende de uma adequada escolha das variáveis instrumentais (MATHIAS, 1998).

Neste trabalho os coeficientes do modelo da classe ARMA serão estimados somente a partir de métodos derivados da aproximação dos mínimos quadrados.

Devido este modelo ser o objeto de pesquisa desse trabalho, uma seção especial será destinada a uma revisão de literatura sobre os principais trabalhos realizados utilizando o modelo ARMA e suas variações na análise de vibração estrutural.

\subsection{REVISÃO DE LITERATURA - MODELO ARMA E SUAS VARIAÇÕES}

O desenvolvimento do modelo ARMA proposto por Wald (1947) inicia sua evolução histórica a partir dos modelos paramétricos auto-regressivo (AR) e de média móvel (MA) desenvolvidos na década de 20 por Yule (1927).

A formulação destes modelos foi fundamentada na teoria estatística com o objetivo de obter equações paramétricas que melhor se ajustassem a um conjunto de dados de análise, tendo sua utilização em vários campos do conhecimento.

Box e Jenkins (1976) desenvolveram uma metodologia, utilizando o modelo misto ARMA, bastante empregada na economia. Esta metodologia baseia-se no resultado de que todos os processos estocásticos estacionários podem ser representados por um modelo linear de média móvel. 
Na década de 60, graças ao surpreendente avanço tecnológico e o surgimento de estimadores cada vez mais robustos, este modelo passou a ser largamente empregado na área de engenharia elétrica, como processamento de filtros digitais e controle digital de sinais.

Já a aplicação dos modelos paramétricos derivados do modelo ARMA no campo da análise de vibrações foi consolidada nos anos 70. A partir dessa época, várias técnicas utilizando o modelo da classe ARMA, têm sido propostas e aplicadas com sucesso na área de análise modal de estrutura.

Um levantamento abrangente dos trabalhos que foram realizados na área de vibração estrutural utilizando o modelo ARMA, segundo aqueles acessados são descritos a seguir:

Em 1973, Gersch, Nielsen e Akaike apresentaram um procedimento para estimar os parâmetros estruturais de um sistema utilizando o modelo ARMA, a partir de dados de vibração aleatória. Neste procedimento, o modelo ARMA é ajustado através da função de correlação dos dados amostrados, calculadas a partir do método de estimação estocástica ML. A confiabilidade estatística dos parâmetros estimados foi medida através do coeficiente de variação, desvio padrão e a média dos parâmetros estimados. No ano seguinte, Gersch (1974) explorou as propriedades do método da ML para determinar um desempenho estatístico teoricamente ótimo dos parâmetros modais estimados de um sistema estrutural.

Gersch e Foutch (1974), motivados pela dificuldade encontrada no método da ML, desenvolveram um procedimento baseado na aproximação dos mínimos quadrados em dois estágios (2SLS) utilizando apenas a função de covariância dos dados observados para estimar os coeficientes do modelo ARMA. A vantagem desse método é que além de manter a eficiência estatística do método da ML, o processo computacional é considerado mais simples e mais econômico. Aplicações dessa versão do método 2SLS na estimativa de freqüências naturais e fatores de amortecimento a partir de dados experimentais obtidos do World Trade Center, Tokyo, Japan, são encontradas em (GERSCH, TAOKA e LUI, 1976).

O trabalho de (YOUNG, 1976) demonstra uma teoria de unificação entre o método IV e uma aproximação do método ML no contexto da estimação da máxima verossimilhança. Com esse estudo tornou-se mais aparente as vantagens e limitações 
dessas duas técnicas; e possíveis procedimentos para obter uma melhor eficiência estatística foram apresentados. A implementação do algoritmo unificado recursivo denominado de IVAML é discutida em detalhes e as propriedades estatísticas são avaliadas empiricamente utilizando análise de simulação de Monte Carlo em (YOUNG e JAKEMAN, 1979).

Davies e Hammond (1984) trazem uma comparação e apresentam as vantagens e desvantagens entre o método da variável instrumental recursiva (RIV, Recursive Instrumental Variable) e o método dos mínimos quadrados recursivo (RLS, Recursive Least-Square). No trabalho de (GHANEM e SHINOZUKA, 1995) é realizada uma revisão de alguns algoritmos de identificação estrutural tais como ML, RLS e RIV. Resultados de aplicações desses algoritmos na identificação de sistemas estruturais, a partir de dados experimentais obtidos em condições laboratoriais controladas, são apresentados em outro trabalho realizado pelos mesmos autores (SHINOZUKA e GHANEM, 1995). Detalhes sobre as técnicas de identificação que utilizam algoritmos recursivos podem ser encontradas em (LJUNG e SÖDEERSTROM, 1983).

Em um trabalho mais recente, Larbi e Lardies (2000) desenvolveram um método de identificação dos parâmetros modais fundamentado no modelo ARMA multivariável que utiliza somente medidas de resposta. Neste método, os coeficientes da parcela autoregressiva, que contém as informações dinâmicas do sistema, são determinados através de um procedimento iterativo baseado na função de máxima verossimilhança.

Um procedimento de identificação de sistemas através do modelo ARMA utilizando a transformada $\mathrm{z}$ foi proposto por Mickleborough e Pi (1989). No mesmo ano os autores contribuíram com o desenvolvimento de um novo método de identificação de parâmetros modais. Neste método, os autovalores de uma estrutura em vibração são identificados através dos pólos de um modelo ARMA, dos quais as freqüências naturais e fatores de amortecimento são determinados. Os coeficientes matriciais do modelo AR multivariável são estimados usando dados de entrada e saída. A matriz de função de resposta ao impulso é então calculada através do relacionamento entre os modelos AR e MA. A função de resposta ao impulso estimada e os pólos do modelo, são então utilizados na identificação dos autovetores e consequentemente os modos de vibrar da estrutura (PI e MICKLEBOROUGH, 1989).

Piombo et al (1993) realizou um estudo com o objetivo de avaliar a variação das características dinâmicas de uma ponte de grande porte sob excitação aleatória causada pelo tráfego. O procedimento utilizado na identificação dos parâmetros modais é 
baseado no modelo ARMAV. A extração dos coeficientes desse modelo é realizada através de um algoritmo dos mínimos quadrados iterativos, o qual divide o problema global em um conjunto de subproblemas mais simples, um para cada grau de liberdade (D’ALESSANDRO et al, 1991). Aplicações do modelo ARMAV na identificação e análise modal de pontes sob excitação ambiente pode ser visto em (GIORCELLI et al, 1994 e GARIBALDI, GIORCELLI e PIOMBO, 1998).

Giorcelli et al (1994) propôs uma técnica para eliminar o problema que surge quando tentamos identificar os parâmetros modais com poucas entradas aleatórias. O modelo ARMAV não é capaz de entender quais são os modos excitados, resultando em estimativas errôneas dos parâmetros. A técnica proposta baseia-se na adição de um novo termo no modelo, tomado como uma entrada exógena. A essa nova representação do modelo dá-se o nome de ARMAX.

O trabalho de (AGUIRRE, RODRIGUES e JÁCOME, 1998) traz uma revisão dos principais tópicos relacionados à identificação de sistemas dinâmicos não lineares e apresenta resultados úteis na seleção da estrutura de modelos NARMAX polinomiais.

Gautier, Gontier e Smail (1995) preocupados em solucionar o problema relacionado com os estimadores tendenciosos, resultantes da aplicação dos métodos no domínio em dados contaminados por ruído, propuseram um método (SISO) direto fundamentado no modelo ARMA, para identificar as propriedades dinâmicas de um sistema, sem a utilização de técnicas de filtragem. Este método baseia-se nas propriedades assintóticas da matriz de covariância dos dados de entrada e saída do sistema, obtidos experimentalmente. Basicamente, o procedimento utilizado tenta encontrar, através de um método não linear, o melhor valor da variância do ruído que pode ser utilizado para corrigir a matriz de covariância.

O trabalho de (FASSOIS e LEE, 1993) aborda o problema da análise modal experimental estocástica onde o sistema dinâmico é representado através de uma forma especial do modelo ARMAX para múltiplas entradas e múltiplas saídas. A técnica de extração dos parâmetros do modelo utiliza um algoritmo linear em múltiplos estágios onde as medidas de respostas podem ser consideradas como deslocamento, velocidade ou aceleração do sistema. Anos depois Fassois (2001) introduz uma técnica MIMO de identificação de estruturas dinâmicas utilizando uma nova versão para aproximação dos mínimos quadrados em múltiplos estágios (LMS), na determinação dos coeficientes do modelo ARMAX. Esta nova versão caracteriza-se por uma modesta complexibilidade computacional, seleção da ordem via propriedades estatísticas, estabilidade do modelo, 
garantindo assim aplicabilidade aos problemas de ordem alta e estruturas com baixo amortecimento e efetiva distinção entre os modos estruturais e espúrios via análise de dispersão digital. É realizada uma avaliação do método considerando a presença de ruído e uma comparação com uma versão mais simples do modelo ARX, e o algoritmo ERA, utilizando dados de vibrações experimental obtidos de uma estrutura aeronáutica. Papakos e Fassois (2003) apresentam uma formulação que utiliza o modelo AR e ARMA estimados respectivamente, através de uma regressão linear e do método LMS desenvolvido recentemente por Fassois (2001). As vantagens e limitações dessa formulação são avaliadas através da identificação das características dinâmicas de um protótipo de estrutura aeronáutica sob excitação não observável. Em geral, os métodos alcançam a mesma precisão, no entanto o método LMS leva à modelos de ordem mais baixa e conseqüentemente um menor número de modos espúrios.

Uma comparação crítica entre os métodos estocásticos tais como o método do erro preditor (PEM, Prediction Error Method), 2SLS, LMS, IV e os métodos determinísticos LS, Prony e ERA, na identificação dos parâmetros modais de estruturas vibrantes obtidos a partir de excitações aleatórias e dados de respostas contaminados por ruídos, é apresentada em (PETSOUNIS e FASSOIS, 2001). Os métodos estocásticos mostram-se mais vantajosos em relação aos métodos determinísticos quando aplicados aos dados corrompidos por ruído, no entanto, apresentam uma maior complexidade teórica e computacional.

Outros estudos comparativos entre os vários métodos de identificação podem ser encontrados na literatura. Dentre os quais menciona-se os trabalhos de (KADAKAL e YÜZÜGÜLLÜ, 1995), que utiliza as aproximações IV e LS para obter os coeficientes do modelo AR, a partir de dados medidos em condição operacional; (DESFORGES, COOPER e WRIGHT1, 1995) onde as estimativas dos parâmetros modais obtidas a partir dos métodos da autocorrelação, decremento aleatório, máxima entropia e mínimos quadrados em dois estágios foram comparadas com estimativas obtidas através do método tradicional de função de resposta em freqüência. Hermans et al, (1997) realiza a identificação a partir de dados de entrada e saída ou apenas saída, obtidos em condição real de carregamento, através do modelo ARV e o método LSCE. Peeters e De Roeck, ( 2001) traz uma revisão entre alguns métodos de identificação em ambos os domínios no âmbito da análise modal operacional, e realiza uma comparação entre os métodos no domínio da freqüência: Pico à Pico (PP, Peak-Picking) e o método da Função indicadora dos Modos Complexos (CMIF, Complex Mode Indication Function), 
considerado uma extensão de PP, onde é empregado a decomposição em valores singulares (SVD), e no domínio do tempo: o método IV, bem como o método de identificação estocástica de subespaço (SSI, Stochastic Subspace Identification); Peeters et al, (1998) apresenta um estudo e comparação entre os métodos de identificação estocástica PP, LSCE e SSI. Os princípios básicos destes métodos são brevemente revisados e os aspectos práticos de suas aplicações em dados de uma ponte em condição de operação são discutidos. Peeters, De Roeck e Andersen, (1999) tratam da identificação estocástica utilizando o método SSI e o método PEM. O primeiro é um método linear, considerado sub-ótimo uma vez que não é baseado num critério de minimização, e o segundo é um método não linear que encontra os parâmetros do modelo ARMA através de um processo iterativo de otimização. As incertezas dos parâmetros modais foram calculadas e comparadas a partir dos resultados obtidos através dos métodos SSI e PEM. Garibaldi et al, (2001) apresenta uma comparação entre o método de identificação baseado no modelo ARMAV (LMS), e o método de análise da variável canônica (CVA, Canonical Variate Analysis). Os parâmetros identificados foram extraídos a partir de dados reais de vibração, causados pelo tráfego de veículos em uma ponte, e pela excitação sísmica em um edifício, causada por um terremoto na Itália em 1997.

Bodeux e Golinval (2001 e 2003) aplicam o modelo ARMAV na detecção de falhas estruturais. Em ambos os trabalhos a estimação dos coeficientes do modelo é realizada através do método PEM, conhecido por proporcionar uma estimativa eficiente e não tendenciosa. A vantagem de utilizar este método é que além de permitir estimar os parâmetros modais é possível também determinar as incertezas dos parâmetros estimados. Com base nessas incertezas é possível avaliar a existência de falhas estruturais e então planejar a manutenção preventiva com segurança, sem que haja prejuízo.

Como pôde ser visto diante dessa revisão bibliográfica, modelos da classe ARMA têm sido largamente estudados e aplicados na análise modal de estruturas. 


\section{(2) Representação de Sistemas Dinâmicos em Espaço de Estado}

\subsection{PRELIMINARES}

No estudo de um sistema é importante conhecer o modelo matemático que descreve seu comportamento. Este capítulo traz a representação matemática de sistemas dinâmicos através do modelo em espaço de estado. Primeiramente é apresentada a modelagem em espaço de estado em tempo contínuo e em seguida, em tempo discreto. Posteriormente, a partir de uma transformação linear entre o vetor das coordenadas modais e o vetor de estado obtém-se o modelo modal, através do qual são extraídas as características dinâmicas do sistema a partir das freqüências naturais, fatores de amortecimento e modos de vibrar.

A representação de um sistema linear, apresentada neste capítulo, através do modelo em espaço de estado, deve-se principalmente, à necessidade de se mostrar a relação de equivalência entre a representação de um sistema no modelo de espaço de estado e o modelo ARMAV, que será apresentada no capítulo quatro. 


\subsection{MODELAGEM EM ESPAÇO DE ESTADO - SISTEMA EM TEMPO CONTÍNUO}

As equações de movimento de um sistema dinâmico com $n$ graus de liberdade (ngdl), linear e invariante no tempo, podem ser representadas através do seguinte sistema de equações diferenciais ordinárias de segunda ordem:

$$
M \ddot{w}(t)+C \dot{w}(t)+K w(t)=f(t)
$$

onde $M, C$ e $K$ são as matrizes de massa, amortecimento e rigidez respectivamente, todas de dimensão $n \times n$. Os vetores, $\ddot{w}(t) \dot{w}(t)$ e $w(t)$ são os vetores aceleração, velocidade e deslocamento, cujas dimensões são $n \times 1$, onde $t$ representa o tempo contínuo. Já $f(t)$ é o vetor de força externa de dimensão $n \times 1$ que atua no sistema.

O vetor força pode ser decomposto da seguinte forma:

$$
f(t)=F u(t)
$$

onde $F$ é a matriz de influência de entrada de dimensão $n \times r$, que caracteriza a localização de entrada, e $u(t)$ é o vetor de entrada de dimensão $r \times 1$, onde $r$ é o número de entradas do sistema.

As equações de movimento que são compostas por $n$ equações diferenciais de segunda ordem podem ser representadas de maneira equivalente através de um sistema de $2 n$ equações diferenciais de primeira ordem, chamado de modelo de espaço de estado.

Este modelo equivalente pode ser obtido considerando a identidade $\dot{w}(t)=\dot{w}(t)$ e pré-multiplicando a equação do movimento por $M^{-1}$. Desta forma tem-se o seguinte sistema:

$$
\left\{\begin{array}{l}
\dot{w}(t)=\dot{w}(t) \\
\ddot{w}(t)=-M^{-1} C \dot{w}(t)-M^{-1} K w(t)+M^{-1} F u(t)
\end{array}\right.
$$




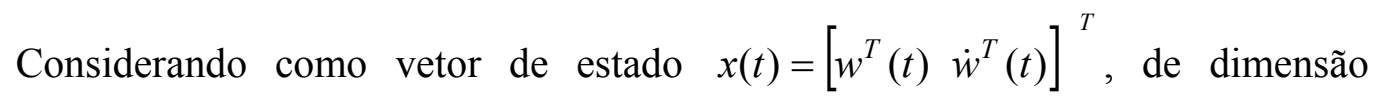
$2 n \times 1$, as $2 n$ equações diferenciais de primeira ordem, denominada equações de estado são dadas por:

$$
\dot{x}(t)=A_{c} x(t)+B_{c} u(t)
$$

onde

$A_{c}=\left[\begin{array}{cc}0 & I \\ -M^{-1} K & -M^{-1} C\end{array}\right] \quad$ é a matriz de estado em tempo contínuo, de dimensão $2 n \times 2 n$.

$B_{c}=\left[\begin{array}{c}0 \\ M^{-1} F\end{array}\right]$ é a matriz de influência de entrada em tempo contínuo, de dimensão $2 n \times r$.

As equações de estado (3.2) modelam o comportamento dinâmico do sistema assim como as $n$ equações diferenciais dadas por (3.1).

Seja o vetor de medidas de saída do sistema de dimensão $m \times 1$ dado da seguinte forma:

$$
y(t)=L_{d} w(t)+L_{v} \dot{w}(t)+L_{a} \ddot{w}(t)
$$

onde $L_{d}, L_{v}$ e $L_{a}$ são as matrizes de influência de saída para o deslocamento, velocidade e aceleração respectivamente, de dimensão $m \times n$.

Utilizando a igualdade

$$
\ddot{w}(t)=-M^{-1} C \dot{w}(t)-M^{-1} K w(t)+M^{-1} F u(t)
$$


e substituindo-a em (3.3) obtém-se

$$
y(t)=\left(L_{d}-L_{a} M^{-1} K\right) w(t)+\left(L_{v}-L_{a} M^{-1} C\right) \dot{w}(t)+\left(L_{a} M^{-1} F\right) u(t)
$$

Definindo

$$
C_{c}=\left[\begin{array}{ll}
\left(L_{d}-L_{a} M^{-1} K\right) \quad\left(L_{v}-L_{a} M^{-1} C\right)
\end{array}\right]
$$

como a matriz de influência de saída no espaço de estado em tempo contínuo, de dimensão $m \times 2 n$, e a matriz de transmissão direta

$$
D_{c}=L_{a} M^{-1} F
$$

de dimensão $m \times r$, a equação (3.4) pode ser reescrita da forma:

$$
y(t)=C_{c} x(t)+D_{c} u(t)
$$

a qual é denominada de equação de saída, ou de observação, uma vez que controla qual parte da saída é observada.

Desta forma, o modelo de espaço de estado em tempo contínuo é dado por:

$$
\left\{\begin{array}{l}
\dot{x}(t)=A_{c} x(t)+B_{c} u(t) \\
y(t)=C_{c} x(t)+D_{c} u(t)
\end{array}\right.
$$

A solução completa da equação de estado em (3.5) é composta pela soma da solução homogênea com a solução particular, para $t>t_{0}$.

Assim, considerando $t_{0}=0$, a solução completa $x(t)=x(t)_{h}+x(t)_{p}$, conforme (CHEN, 1999), é dada por 


$$
x(t)=e^{A_{c} t} x(0)+\int_{0}^{t} e^{A_{c}(t-\tau)} B_{c} u(\tau) d \tau
$$

onde a matriz $e^{A_{c} t}$ corresponde a série convergente:

$$
e^{A_{c} t}=I+t A_{c}+\frac{t^{2}}{2 !} A_{c}^{2}+\cdots=\sum_{k=0}^{\infty} \frac{1}{k !} t^{k} A_{c}{ }^{k}
$$

Substituindo (3.6) na equação de saída de (3.5) obtém-se:

$$
y(t)=C_{c} e^{A_{c} t} x(0)+C_{c} \int_{0}^{t} e^{A_{c}(t-\tau)} B_{c} u(\tau) d \tau+D_{c} u(t)
$$

\subsection{MODELAGEM EM ESPAÇO DE ESTADO - SISTEMA EM TEMPO DISCRETO}

Nos procedimentos experimentais as medidas são representadas por sinais discretos no domínio do tempo. Assim, se faz necessário à representação do modelo em espaço de estado em tempo discreto. Isso é feito considerando as variáveis medidas em intervalos de tempo igualmente espaçados de $\Delta t \operatorname{com} t=0, \Delta t, \ldots, k \Delta t,(k+1) \Delta t$, ...(l-1) $\Delta t$.

A solução da equação de estado em tempo contínuo, dada em (3.6), pode ser representada em tempo discreto reescrevendo-a no instante de tempo $t=k \Delta t$ e $t=(k+1) \Delta t$, e assumindo que o vetor de entrada seja constante, ou seja, $u(\tau)=u(k \Delta t)=u(k)$ para $k \Delta t \leq \tau \leq(k+1) \Delta t$. Desta forma tem-se: 


$$
\begin{gathered}
x(k)=x(k \Delta t)=e^{A_{c} k \Delta t} x(0)+\int_{0}^{k \Delta t} e^{A_{c}(k \Delta t-\tau)} B_{c} u(\tau) d \tau \\
\mathrm{e} \quad x(k+1)=x((k+1) \Delta t)=e^{A_{c}(k+1) \Delta t} x(0)+\int_{0}^{(k+1) \Delta t} e^{A_{c}((k+1) \Delta t-\tau)} B_{c} u(\tau) d \tau
\end{gathered}
$$

A equação logo acima pode ser reescrita da seguinte forma:

$$
x(k+1)=e^{A_{c} \Delta t}\left[e^{A_{c} k \Delta t} x(0)+\int_{0}^{k \Delta t} e^{A_{c}(k \Delta t-\tau)} B_{c} u(\tau) d \tau\right]+\int_{k \Delta t}^{(k+1) \Delta t} e^{A_{c}(k \Delta t+\Delta t-\tau)} B_{c} u(\tau) d \tau
$$

Fazendo uma substituição de variável de $\tau$ para $\alpha$, onde $\alpha=k \Delta t+\Delta t-\tau$ obtém-se:

$$
x(k+1)=e^{A_{c} \Delta t} x(k)+\int_{0}^{\Delta t} e^{A_{c} \alpha} d \alpha B_{c} u(k)
$$

Definindo a matriz de estado e a matriz de influência de entrada em tempo discreto da seguinte forma:

$$
\begin{gathered}
A_{d}=e^{A_{c} \Delta t} \\
B_{d}=\left(\int_{0}^{\Delta t} e^{A_{c} \alpha} d \alpha\right) B_{c}
\end{gathered}
$$

a equação (3.7) pode ser escrita como

$$
x(k+1)=A_{d} x(k)+B_{d} u(k)
$$


As relações entre as matrizes do modelo discreto e contínuo são dadas por:

$$
\begin{gathered}
A_{c} \Delta t=\ln A_{d} \Leftrightarrow A_{c}=\frac{1}{\Delta t} \ln A_{d} \\
B_{d}=\left[e^{A_{c} \Delta t}-I\right] A_{c}^{-1} B_{c}=\left[A_{d}-I\right] A_{c}^{-1} B_{c} \\
C_{d}=C_{c} \\
D_{d}=D_{c}
\end{gathered}
$$

Desta forma, o modelo de espaço de estado em tempo discreto é dado pelas equações:

$$
\left\{\begin{array}{c}
x(k+1)=A_{d} x(k)+B_{d} u(k) \\
y(k)=C_{d} x(k)+D_{d} u(k)
\end{array}\right.
$$

Através de substituições recursivas da equação de estado na equação de saída em (3.9) nos instantes de tempo $k+1, k+2, \cdots, k+l+1$ tem-se :

$$
\begin{aligned}
x(k+2)= & A_{d} x(k+1)+B_{d} u(k+1)=A_{d}{ }^{2} x(k)+A_{d} B_{d} u(k)+B_{d} u(k+1) \\
y(k+1)= & C_{d} x(k+1)+D_{d} u(k+1)=C_{d} A_{d} x(k)+C_{d} B_{d} u(k)+D_{d} u(k+1) \\
x(k+3)= & A_{d} x(k+2)+B_{d} u(k+2)=A_{d}{ }^{3} x(k)+A_{d}{ }^{2} B_{d} u(k)+A_{d} B_{d} u(k+1)+B_{d} u(k+2) \\
y(k+2)= & C_{d} x(k+2)+D_{d} u(k+2)=C_{d} A_{d}{ }^{2} x(k)+C_{d} A_{d} B_{d} u(k)+C_{d} B_{d} u(k+1)+ \\
& D_{d} u(k+2) \\
x(k+4)= & A_{d} x(k+3)+B_{d} u(k+3)=A_{d}{ }^{4} x(k)+A_{d}{ }^{3} B_{d} u(k)+A_{d}{ }^{2} B_{d} u(k+1)+ \\
& A_{d} B_{d} u(k+1)+A_{d} B_{d} u(k+2)+B_{d} u(k+3) \\
y(k+3)= & C_{d} x(k+3)+D_{d} u(k+3)=C_{d} A_{d}{ }^{4} x(k)+C_{d} A_{d}{ }^{3} B_{d} u(k)+C_{d} A_{d} B_{d} u(k+1)+ \\
& B_{d} u(k+2)+D_{d} u(k+3) \\
& \vdots
\end{aligned}
$$




$$
\begin{aligned}
& x(k+l+1)=A_{d}{ }^{l+1} x(k)+\sum_{j=1}^{l+1} A_{d}^{l+1-j} B_{d} u(k+j-1) \\
& y(k+l)=C_{d} A_{d}{ }^{l} x(k)+\sum_{j=1}^{l} C_{d} A_{d}{ }^{l-j} B_{d} u(k+j-1)+D_{d} u(k+l)
\end{aligned}
$$

onde os dois últimos termos da seqüência acima representam o vetor de estado e o vetor de saída em instantes de tempo $k+l$, para $l \geq 0$.

A equação (3.10-b) representa a solução de (3.9), que é composta pela solução homogênea e solução particular, descritas pela primeira e segunda parcela, respectivamente.

\subsection{MODELO DE ESTADO NAS COORDENADAS MODAIS}

Através de uma transformação linear entre as coordenadas modais e as variáveis de estado, obtém-se uma representação desacoplada do modelo em espaço de estado, denominado de modelo modal.

Para tanto, define-se a matriz modal da seguinte forma:

$$
\Psi=\left[\begin{array}{cccccccc}
\phi_{1} & \phi_{2} & \cdots & \phi_{n} & \phi_{1}^{*} & \phi_{2}^{*} & \cdots & \phi_{n}^{*} \\
\lambda_{1} \phi_{1} & \lambda_{2} \phi_{2} & \cdots & \lambda_{n} \phi_{n} & \lambda_{1}^{*} \phi_{1}^{*} & \lambda_{2}^{*} \phi_{2}^{*} & \cdots & \lambda_{n}^{*} \phi_{n}^{*}
\end{array}\right]
$$

com dimensão $2 n \times 2 n$, onde cada coluna de $\Psi$ corresponde a um autovetor $\psi_{j}$ da matriz $A_{d}$, com seu respectivo autovalor $\lambda_{j}$ para $j=1, \cdots, 2 n$. A partir da matriz modal é definida a seguinte transformação linear:

$$
x(k)=\Psi x(k)_{M}
$$


onde $\Psi$ é a matriz de transformação, $x(k)$ é o vetor de estado e $x(k)_{M}$ o vetor das coordenadas modais. Substituindo a equação (3.11) em (3.9) e pós multiplicando-a por $\Psi^{-1}$ obtém-se um novo modelo de estado escrito em termos das coordenadas modais:

$$
\left\{\begin{aligned}
x(k+1)_{M} & =A_{M} x(k)_{M}+B_{M} u(k) \\
y(k) & =C_{M} x(k)_{M}+D_{M} u(k)
\end{aligned}\right.
$$

com

$$
A_{M}=\Psi^{-1} A_{d} \Psi \quad B_{M}=\Psi^{-1} B_{d} \quad C_{M}=C_{d} \Psi \quad D_{M}=D_{d}
$$

A matriz $A_{M}$ é uma matriz diagonal de dimensão $2 n \times 2 n$ constituída por autovalores de $A_{d}$, e pode ser denotada por $A_{M}=\lambda=\operatorname{diag}\left\{\lambda_{j}\right\} \operatorname{com} j=1,2, \cdots, 2 n$.

De (3.13) obtém-se a decomposição modal da matriz $A_{d}$ :

$$
A_{d}=\Psi \lambda \Psi^{-1}
$$

A matriz de influência de entrada nas coordenadas modais $B_{M}$ de dimensão $2 n \times r$ é formada por elementos que definem a amplitude relativa com que cada modo é excitado através de uma determinada entrada.

Através do relacionamento entre as matrizes $A_{c}$ e $A_{d}$ dado pela equação (3.8) verifica-se que seus autovalores se relacionam através da expressão:

$$
\text { autovalor }\left(A_{c}\right)=\frac{1}{\Delta t} \text { autovalor }\left(\ln \left(A_{d}\right)\right)
$$

Uma vez que neste trabalho os sistemas considerados possuem fatores de amortecimento sub-amortecido $\zeta<1$, os autovalores apresentam-se em $n$ pares complexos conjugados. Desta forma, os pares de autovalores da matriz $A_{c}$ são definidos por: 


$$
\alpha_{j}=-\sigma_{j} \pm i \omega d r_{j} \quad j=1, \cdots, n
$$

onde, para sistemas com amortecimento proporcional, tem-se que o amortecimento modal $\sigma_{j}=-\zeta_{j} \omega_{j}$ é a parte real com $\sigma_{j}>0$, e a freqüência natural amortecida $\omega d r_{j}=\omega_{j} \sqrt{1-\zeta_{j}^{2}}$ é a parte imaginária do autovalor $\alpha_{j}$. Através da relação (3.15), obtém-se que:

$$
\begin{gathered}
\alpha_{j}=\frac{\ln \lambda_{j}}{\Delta t} \\
\lambda_{j}=e^{\alpha_{j} \Delta t}
\end{gathered}
$$

onde $\lambda_{j}$ é o autovalor de $A_{d}$. Desta forma, através das equações (3.16) e (3.17) as freqüências naturais $\omega_{j}$ e os fatores de amortecimento $\zeta_{j}$ são determinados por:

$$
\omega_{j}=\frac{\left|\alpha_{j}\right|}{2 \pi}(\mathrm{Hz}) \quad \text { e } \quad \zeta_{j}=-\frac{\operatorname{Re}\left(\alpha_{j}\right)}{\left|\alpha_{j}\right|}
$$

Na equação de observação (3.12-b), $C_{M}$ é a matriz de influência de saída de dimensão $m \times 2 n$, cujas colunas contém informação da forma dos modos conforme a localização espacial dos sensores.Assim a matriz modal $\Phi$ é obtida por:

$$
\Phi=C_{M}=C_{d} \Psi=\left[\begin{array}{llll}
\phi_{1} & \phi_{2} \cdots \phi_{n} & \phi_{1}^{*} & \phi_{2}^{*} \cdots \phi_{n}^{*}
\end{array}\right]
$$

onde $*$ denota o modo complexo conjugado.

O conjunto de matrizes $A_{d}, B_{d}$ e $C_{d}$ é denominado de realização do sistema. A realização dada pela equação (3.12) é um caso especial onde se utilizou a transformação linear (3.11) tomando a matriz modal como matriz de transformação e as coordenadas modais como variáveis de estado. 
Ressalte-se, porém, que tal realização não é única, sendo possível encontrar para uma infinidade de matrizes de transformação não singulares e de vetores de estado, uma nova realização através de transformações de similaridades.

Qualquer que seja a realização obtida a partir de uma transformação de similaridade, deve representar o mesmo comportamento dinâmico global, ou seja, deve possuir os mesmos autovalores.

Uma realização para ser ideal, deve ter uma dimensão de espaço de estado mínima, onde todos os modos do sistema possam ser excitados, através de pontos adequados na estrutura, e observados na saída do sistema. Uma realização é dita mínima, somente se a mesma for observável e controlável.

Teorema 3.1. Um sistema em espaço de estado de dimensão $2 n$ é observável e controlável se e somente se a matriz de observabilidade $Q_{o}(v)$ de dimensão $m v \times 2 n$, e a matriz de controlabilidade $Q_{c}(v)$ de dimensão $2 n \times v r$ definidas como:

$$
Q_{o}(v)=\left[\begin{array}{c}
C_{d} \\
C_{d} A_{d} \\
\vdots \\
C_{d} A_{d}{ }^{\nu-1}
\end{array}\right] \quad \text { e } \quad Q_{c}(v)=\left[\begin{array}{lll}
B_{d} & A_{d} B_{d} & \cdots A_{d}{ }^{v-1} B_{d}
\end{array}\right]
$$

possuem posto $2 n$.

O conceito de controlabilidade está relacionado com a forma, com que os modos da estrutura são excitados pelo sinal de entrada; enquanto que a observabilidade é um parâmetro de avaliação de como cada modo excitado contribui na saída do sistema.

No caso da observabilidade, se existir por exemplo, somente um sensor de medida de resposta, e esse sensor estiver localizado sobre a linha nodal de um modo, então não haverá contribuição desta coordenada modal na saída do sistema, ou seja, este modo não será observável.

Já no caso da controlabilidade, se existir apenas um excitador e este for acoplado em uma linha nodal de um certo modo, isso significa que a entrada não conseguirá excitar essa coordenada modal e portanto esse modo não será controlável. 
Uma forma de aumentar a observabilidade do vetor de saída e a controlabilidade do vetor de estado é a utilização de uma quantidade maior de sensores e excitadores, respectivamente.

Uma explicação mais abrangente sobre esses conceitos pode ser vista em (JUANG, 1994). 


\section{Desenvolvimento Teórico da Relação de Equivalência entre - Modelo de Espaço de Estado e o Modelo ARMAV}

\subsection{PRELIMINARES}

Um sistema linear pode ser representado sob a forma de equação de diferença, função de transferência ou através da equação de espaço de estado, sendo esta última, vista no capítulo anterior.

O modelo ARMAV, utilizado neste trabalho, é uma forma especial de representar um sistema através de uma equação de diferença, onde a saída para um dado instante $k$ pode ser representada como função das entradas até o instante do tempo de análise e das saídas passadas. Este capítulo tem como objetivo principal apresentar em detalhes, através de um desenvolvimento teórico, a relação de equivalência que existe entre o modelo de espaço de estado e o modelo ARMAV (ANDERSEN, 1997). É apresentada a modelagem tanto para sistemas determinísticos quanto para sistemas estocásticos. No modelo determinístico as entradas e as saídas do sistema são livres de ruído, o que permite que a saída para qualquer instante de tempo possa ser determinada 
se os estados iniciais são conhecidos. Porém para os modelos estocásticos é possível somente predizer a resposta do sistema, uma vez que a entrada não é conhecida, mas apenas modelada implicitamente através do ruído.

\subsection{MODELAGEM DE SISTEMAS DETERMINÍSTICO E ESTOCÁSTICO}

Neste trabalho o sistema (3.9) representado através do modelo de estado será adotado como um sistema determinístico, uma vez que a entrada $u(k)$ é suposta conhecida. Assume-se que o modelo:

$$
\left\{\begin{array}{c}
x(k+1)=A_{d} x(k)+B_{d} u(k) \\
y(k)=C_{d} x(k)+D_{d} u(k)
\end{array}\right.
$$

é excitado através de um processo ruído branco gaussiano de média zero e estacionário. Em outras palavras isso quer dizer que:

$$
\begin{aligned}
& \mathrm{E}[u(k)]=0 \\
& \mathrm{E}\left[u(k) u^{T}(k+j)\right]=\Delta \delta(j), \quad j=0,1,2, \cdots
\end{aligned}
$$

onde $E[\cdot]$ representa o operador esperança, $\delta(j)$ é o delta de Kronecker e $\Delta$ matriz de covariância do processo ruído branco $u(k)$.

Na prática, geralmente além da entrada $u(k)$ do sistema (4.1), existem outras entradas que de uma forma não controlada contribuem para a resposta do sistema. Essas influências indesejáveis são caracterizadas por distúrbios causados por diferentes fenômenos tais como: ruídos gerados por sensores; pequenas não linearidades no modelo ou até mesmo uma excitação não estacionária. 
É razoável, portanto, assumir que a maior parte dos sistemas reais são de natureza estocástica, uma vez que, com a adição desses distúrbios a entrada passa a ser conhecida somente no sentido estatístico.

Os diferentes tipos de distúrbios podem ser divididos em duas categorias:

- Ruído do processo: representa todas as fontes de ruídos que levam a um modelo de espaço de estado linear inadequado.

- Ruído de medida: modela todas as fontes de ruídos que perturbam as medidas de resposta.

Assume-se que o ruído de processo e o ruído de medida possam ser descritos por processos de ruído branco gaussiano de média zero $s(k)$ de dimensão $r \times 1$ e $v(k)$ de dimensão $m \times 1$, respectivamente, com matrizes de covariância dadas por:

$$
E\left[\begin{array}{l}
s(k) \\
v(k)
\end{array}\right]=0 \quad E\left[\left[\begin{array}{l}
s(k) \\
v(k)
\end{array}\right]\left[\begin{array}{ll}
S^{T}(k+j) & v^{T}(k+j)
\end{array}\right]\right]=\left[\begin{array}{cc}
Q & S \\
S^{T} & R
\end{array}\right] \delta(j)
$$

Adicionando as componentes estocásticas, que são o ruído de processo $s(k)$ na equação de estado e o ruído de medida $v(k)$ na equação de observação do modelo (4.1) obtém-se o seguinte modelo determinístico-estocástico:

$$
\left\{\begin{array}{c}
x(k+1)=A_{d} x(k)+B_{d} u(k)+s(k) \\
y(k)=C_{d} x(k)+D_{d} u(k)+v(k)
\end{array}\right.
$$

Nestes sistemas contaminados por ruídos é possível apenas predizer a resposta do sistema.

Para obter um sistema puramente estocástico considera-se a entrada $u(k)=0$ na equação (4.4):

$$
\left\{\begin{array}{c}
x(k+1)=A_{d} x(k)+s(k) \\
y(k)=C_{d} x(k)+v(k)
\end{array}\right.
$$

Neste caso, o sistema é identificado somente através da medida de resposta, e como a entrada não é medida a influência da excitação está implicitamente modelada nos ruídos $s(k)$ e $v(k)$. 


\subsection{DESCRIÇÃO DO MODELO ARMAV}

O comportamento dinâmico de um sistema linear discreto e invariante no tempo pode ser descrito através de um modelo paramétrico de ordem $p$, denominado $\operatorname{ARMAV}(p, p)$. Este modelo descrito através de uma série temporal é definido pela seguinte equação de diferenças matricial (MARPLE, 1987 e KAY, 1987):

$$
y(k)+A_{1} y(k-1)+\cdots+A_{p} y(k-p)=B_{0} u(k)+B_{1} u(k-1)+\cdots+B_{p} u(k-p)
$$

onde $y(k)$ e $u(k)$ representam os vetores de entrada e saída do sistema, respectivamente, em cada instante de tempo $k$. A entrada $u(k)$ é considerada um processo ruído branco, ou seja, um processo de média zero, estacionário e não correlacionado.

Neste modelo a resposta do sistema é escrita como combinação linear das entradas passadas e presentes e saídas passadas, ponderadas pelos coeficientes matriciais $A_{i}$ de dimensões $m \times m$ e $B_{i}$ de dimensões $m \times r$, que representam os termos auto-regressivo (AR) e de média móvel (MA), respectivamente. O termo autoregressivo descreve a dinâmica do sistema, enquanto que o termo de média móvel está relacionado com a excitação.

O modelo $\operatorname{ARMAV}(p, p)$ pode também ser definido em termos da função de transferência, através da aplicação do operador de atraso $\left(q^{-i} y(k)=y(k-i)\right)$ na equação (4.6), resultando em:

$$
y(k)=A^{-1}(q) B(q) u(k)=H(q) u(k)
$$

com

$$
\begin{gathered}
A(q)=I+A_{1} q^{-1}+A_{2} q^{-2}+\cdots+A_{p} q^{-p} \\
B(q)=B_{0}+B_{1} q^{-1}+B_{2} q^{-2}+\cdots+B_{p} q^{-p}
\end{gathered}
$$


onde $A(q)$ e $B(q)$ são polinômios matriciais dos coeficientes auto-regressivo e médiamóvel, respectivamente. Esses polinômios matriciais podem ser obtidos de forma análoga, através da aplicação da transformada $\mathrm{z}$ na equação de diferenças.

\subsection{RELAÇÃO ENTRE O MODELO ARMAV E O MODELO DE ESPAÇO DE ESTADO}

Com o modelo $\operatorname{ARMAV}(p, p)$ definido, o próximo passo é demonstrar através de algumas relações e teoremas a equivalência existente entre este modelo e o modelo de espaço de estado.

Para tanto, considere a resposta do sistema $y(k)$ descrita através de uma parte homogênea, que consiste de um polinômio matricial de ordem $p$, com coeficientes autoregressivos $A_{i}$, com $i=1, \cdots, p$, e uma parte não homogênea, representada através de um polinômio matricial de mesma ordem, com coeficientes de médias móveis $B_{i}$. A solução geral de (4.6) para $k \geq 0$ pode ser representada através da seguinte forma:

$$
y(k)=y_{h}(k)+y_{p}(k)
$$

onde $y_{p}(k)$ é uma solução particular da equação (4.6) e $y_{h}(k)$ consiste numa solução geral da equação homogênea

$$
y(k)+A_{1} y(k-1)+\cdots+A_{p} y(k-p)=0
$$

Uma vez que, no caso da equação homogênea, $y(k)$ pode ser sequencialmente calculada por:

$$
y(k)=-A_{1} y(k-1)-\cdots-A_{p} y(k-p)
$$

o conjunto de soluções das equações homogêneas forma um espaço linear de dimensão pm , ou seja, a solução geral da equação (4.9) depende de pm variáveis independentes. 
A solução geral de (4.6) é portanto dada por (3.10-b) para um vetor de estado $x(k)$ de dimensão $2 n$ onde $2 n=p m$, (GOHBERG, LANCASTER e RODMAN,1982 ).

A parte que consiste da solução homogênea da equação (3.10 b) é dada por:

$$
y_{h}(k+l)=C_{d} A_{d}^{l} x(k)
$$

com $l=0,1, \cdots$.

Inserindo (4.10) na equação (4.9) e pós- multiplicando-a por $A_{d}{ }^{p}$ obtém-se:

$$
\left[C_{d} A_{d}^{p}+A_{1} C_{d} A_{d}^{p-1}+\cdots+A_{p-1} C_{d} A_{d}+A_{p} C_{d}\right] x(k)=0
$$

para qualquer escolha de $x(k)$, se:

$$
C_{d} A_{d}^{p}+A_{1} C_{d} A_{d}^{p-1}+\cdots+A_{p-1} C_{d} A_{d}+A_{p} C_{d}=0
$$

Esta equação (4.11) mostra uma importante relação, que será utilizada no Teorema 4.1, entre as matrizes dos coeficientes auto-regressivos $A_{i}$ e as matrizes de estado $A_{d}$ e de influência de saída $C_{d}$.

Teorema 4.1. Uma realização mínima do sistema em espaço de estado dado por (4.1), descrito pelas matrizes $\left\{A_{d}, B_{d}, C_{d}, D_{d}, \Delta\right\}$ pode ser representada por um modelo $\operatorname{ARMAV}(p, p)$ definido por

$$
y(k)+A_{1} y(k-1)+\cdots+A_{p} y(k-p)=B_{0} u(k)+B_{1} u(k-1)+\cdots+B_{p} u(k-p)
$$


se a realização de espaço de estado satisfaz a exigência dimensional que a dimensão do vetor de estado $2 n$ dividido pelo número de sensores de saída $m$ seja igual a um inteiro $p$, onde $p$ é a ordem do modelo ARMAV, isto é, $(2 n / m)=p$. As $p$ matrizes dos coeficientes auto-regressivos de dimensão $m$ x $m$, são então dadas por:

$$
\left[\begin{array}{lllll}
A_{p} & A_{p-1} & \cdots & A_{2} & A_{1}
\end{array}\right]=-C_{d} A_{d}^{p} Q_{o}^{-1}(p)
$$

As $p+1$ matrizes de coeficientes de médias móveis, de dimensão $m \times \mathrm{x} r$ são dadas por:

$$
\left[\begin{array}{lllll}
B_{p} & B_{p-1} & \cdots & B_{1} & B_{0}
\end{array}\right]=\left[\begin{array}{lllll}
A_{p} & A_{p-1} & \cdots & A_{1} & I
\end{array}\right] T(p+1)
$$

com

$$
T(p+1)=\left[\begin{array}{ccccc}
D_{d} & 0 & \cdots & 0 & 0 \\
C_{d} B_{d} & D_{d} & \cdots & 0 & 0 \\
\vdots & \vdots & \cdots & \vdots & \vdots \\
C_{d} A_{d}{ }^{p-2} B_{d} & C_{d} A_{d}^{p-3} B_{d} & \cdots & D_{d} & 0 \\
C_{d} A_{d}{ }^{p-1} B_{d} & C_{d} A_{d}{ }^{p-2} B_{d} & \cdots & C_{d} B_{d} & D_{d}
\end{array}\right]
$$

onde nota-se que $B_{0}=D_{d}$, e também que a entrada $u(k)$ não é interferida por esta transformação.

\section{Prova do Teorema 4.1:}

Através da equação (4.11) e a da matriz de observabilidade $Q_{o}$, definida no Teorema 3.1, obtém-se a seguinte relação:

$$
\left[\begin{array}{lllll}
A_{p} & A_{p-1} & \cdots & A_{2} & A_{1}
\end{array}\right] Q_{o}(p)=-C_{d} A_{d}^{p}
$$

Pós multiplicando ambos os membros da equação (4.15) por $Q_{o}^{-1}(p)$ : 


$$
\left[\begin{array}{lllll}
A_{p} & A_{p-1} & \cdots & A_{2} & A_{1}
\end{array}\right]=-C_{d} A_{d}^{p} Q_{o}^{-1}(p)
$$

observa-se que os coeficientes matriciais auto-regressivos $A_{i}$ podem ser determinados da realização em espaço de estado, onde $Q_{o}(p)$ é não singular.

Combinando a equação de observação do modelo em espaço de estado (4.1) e a solução geral dada por (3.10-b), os coeficientes matriciais de médias móveis $B_{i}$ são encontrados a partir da construção do seguinte sistema de equações:

$$
\begin{aligned}
& {\left[\begin{array}{c}
y(k-p) \\
y(k-p+1) \\
\vdots \\
y(k-1) \\
y(k)
\end{array}\right]=\left[\begin{array}{c}
C_{d} \\
C_{d} A_{d} \\
\vdots \\
C_{d} A_{d}^{p-1} \\
C_{d} A_{d}^{p}
\end{array}\right] x(k-p)+} \\
& {\left[\begin{array}{ccccc}
D_{d} & 0 & \cdots & 0 & 0 \\
C_{d} B_{d} & D_{d} & \cdots & 0 & 0 \\
\vdots & \vdots & \cdots & \vdots & \vdots \\
C_{d} A_{d}^{p-2} B_{d} & C_{d} A_{d}^{p-3} B_{d} & \cdots & D_{d} & 0 \\
C_{d} A_{d}^{p-1} B_{d} & C_{d} A_{d}^{p-2} B_{d} & \cdots & C_{d} B_{d} & D_{d}
\end{array}\right]\left[\begin{array}{c}
u(k-p) \\
u(k-p+1) \\
\vdots \\
u(k-1) \\
u(k)
\end{array}\right] }
\end{aligned}
$$

onde a primeira equação é a de observação e as $p$ demais representam a solução geral.

O sistema de equações dado por (4.17) na forma compactada pode ser escrito por:

$$
Y(k-p, k)=Q_{o}(p+1) x(k)+T(p+1) U(k-p, k)
$$

Agora introduzindo uma matriz identidade na matriz que contém os coeficientes matriciais auto-regressivos define-se a seguinte matriz:

$$
P=\left[\begin{array}{llllll}
A_{p} & A_{p-1} & \cdots & A_{2} & A_{1} & I
\end{array}\right]
$$


Pré-multiplicando a equação (4.18) por $P$, obtém-se:

$$
\begin{aligned}
P Y(k-p, k) & =P Q_{o}(p+1) x(k)+P T(p+1) U(k-p, k) \\
& =P T(p+1) U(k-p, k)
\end{aligned}
$$

uma vez que reescrevendo a equação (4.11) em termos de $P$ e $Q_{o}(p+1)$, temse $P Q_{o}(p+1)=0$.

O lado esquerdo da equação (4.19) é exatamente a parte auto-regressiva da equação de diferença matricial, enquanto que o termo do lado esquerdo é um polinômio matricial de ordem $p$. Desde que $u(k)$ é um ruído branco com distribuição gaussiana, este polinômio matricial representa o termo de média móvel, o que leva a concluir que a equação (4.19) realmente representa um modelo $\operatorname{ARMAV}(p, p)$.

Definindo os coeficientes matriciais de médias móveis por $B_{0}, B_{1}, \cdots, B_{p}$, segue que:

$$
\left[\begin{array}{lllll}
B_{p} & B_{p-1} & \cdots & B_{1} & B_{0}
\end{array}\right]=\left[\begin{array}{lllll}
A_{p} & A_{p-1} & \cdots & A_{1} & I
\end{array}\right] T(p+1)
$$

Da relação acima pode-se concluir que $D_{d}=B_{0}$.

Pode-se concluir que qualquer sistema em espaço de estado, onde a dimensão do espaço de estado $2 n$ dividido pelo número de saídas observadas $m$ é igual a ordem do modelo ARMAV p, pode ser representado através do modelo ARMAV se a matriz de observabilidade $Q_{o}(p)$ é não singular. A equivalência existente entre o modelo ARMAV e o modelo em espaço de estado é independente da realização utilizada, ou seja, não importa como um sistema linear discreto e invariante no tempo sujeito a uma excitação ruído branco com distribuição gaussiana é realizado; o modelo ARMAV será sempre o mesmo. 


\subsection{UMA REALIZAÇÃO EM ESPAÇO DE ESTADO DO MODELO ARMAV}

Para converter um modelo ARMAV para espaço de estado faz-se necessário a escolha de uma realização. Há várias formas de realizar essa conversão, no entanto, cuidados devem ser tomados nessa escolha de maneira que a realização seja bem condicionada numericamente e de fácil manipulação algébrica em sua construção. Nesta seção será apresentada uma realização de espaço de estado, que é relativamente fácil de ser obtida a partir dos coeficientes matriciais do modelo ARMAV. Esta realização que é construída com base nas matrizes dos coeficientes auto-regressivos e na função de resposta ao impulso do modelo ARMAV é denominada de observabilidade canônica.

\subsubsection{FUNÇÃO DE RESPOSTA AO IMPULSO DO MODELO ARMAV}

Nesta seção serão apresentadas algumas relações envolvendo a função de resposta ao impulso do modelo ARMAV, que serão utilizadas para construir a realização de espaço de estado observabilidade canônica.

O modelo $\operatorname{ARMAV}(p, p)$ pode ser definido equivalentemente como uma convolução da função de resposta ao impulso $h(j)$ e da entrada ruído branco com distribuição gaussiana $u(k)$

$$
y(k)=\sum_{j=0}^{\infty} h(j) u(k-j)
$$

A função transferência definida em (4.7) pode também ser expressa em termos da função de resposta ao impulso e o operador de atraso como:

$$
H(q)=\sum_{j=0}^{\infty} h(j) q^{-j}
$$


De (4.7), tem-se a seguinte relação:

$$
A^{-1}(q) B(q) u(k)=H(q) u(k)
$$

Pré-multiplicando a equação (4.22) por $A(q)$ e substituindo $H(q)$ dado pela equação (4.21) obtém-se:

$$
\left[I+A_{1} q^{-1}+A_{2} q^{-2}+\cdots+A_{p} q^{-p}\right]\left[\sum_{j=0}^{\infty} h(j) q^{-j} u(k)\right]=\left[B_{0}+B_{1} q^{-1}+\cdots+B_{p} q^{-p}\right] u(k)
$$

Comparando os coeficientes de potência de $q$ iguais, o seguinte relacionamento entre as matrizes dos coeficientes do modelo ARMAV e a função de resposta ao impulso $h(j)$, para $j=0, \cdots, p$, pode ser obtido:

$$
\begin{aligned}
& h(0)=B_{0} \\
& h(1)+A_{1} h(0)=B_{1} \\
& h(2)+A_{1} h(1)+A_{2} h(0)=B_{2} \\
& h(3)+A_{1} h(2)+A_{2} h(1)+A_{3} h(0)=B_{3} \\
& h(4)+A_{1} h(3)+A_{2} h(2)+A_{3} h(1)+A_{4} h(0)=B_{4} \\
& \vdots \\
& h(p)+A_{1} h(p-1)+A_{2} h(p-2)+A_{3} h(p-3)+\cdots+A_{p} h(0)=B_{p}
\end{aligned}
$$

Baseado em (4.23) as seguintes relações recursivas podem ser derivadas

$$
\begin{array}{lr}
h(j)=-A_{1} h(j-1)-\cdots-A_{p} h(0)+B_{j}, & 0 \leq j \leq p \\
h(j)=-A_{1} h(j-1)-\cdots-A_{p} h(j-p), & j>p
\end{array}
$$

As últimas $p$ equações de (4.23) podem ser rearranjadas na seguinte forma matricial: 


$$
\left[\begin{array}{c}
h(1) \\
h(2) \\
h(3) \\
h(4) \\
\vdots \\
h(p)
\end{array}\right]=\left[\begin{array}{cccccc}
I & 0 & 0 & \cdots & 0 & 0 \\
A_{1} & I & 0 & \cdots & 0 & 0 \\
A_{2} & A_{1} & I & \cdots & 0 & 0 \\
A_{3} & A_{2} & A_{1} & I & \vdots & \vdots \\
\vdots & \vdots & \vdots & \vdots & \ddots & \vdots \\
A_{p-1} & A_{p-2} & \cdots & \cdots & A_{1} & I
\end{array}\right]^{-1}\left[\begin{array}{c}
B_{1}-A_{1} B_{0} \\
B_{2}-A_{2} B_{0} \\
B_{3}-A_{3} B_{0} \\
B_{4}-A_{4} B_{0} \\
\vdots \\
B_{p}-A_{p} B_{0}
\end{array}\right]
$$

No Teorema 4.2 será mostrado que a matriz de influência de entrada da realização em espaço de estado do modelo $\operatorname{ARMAV}(p, p)$ é dada por $B_{d}=\left[h^{T}(1) h^{T}(2) h^{T}(3) \cdots h^{T}(p)\right]^{T}$.

\subsubsection{A REALIZAÇAO EM ESPAÇO DE ESTADO - OBSERVABILIDADE CANÔNICA}

Para mostrar a realização em espaço de estado é necessário que seja definido um preditor de resposta ótimo, o qual será utilizado no desenvolvimento teórico a seguir.

Para tanto, define-se como preditor ótimo, o preditor de um passo a frente $\hat{y}(k \mid k-1)$ da resposta $y(k)$, como sendo a média condicional de $y(k)$, dada todas as medidas anteriores, $\quad y(0), y(1), \cdots, y(k-1), \quad$ coletadas em um vetor

$$
\begin{aligned}
Y^{k-1}=\{y(0), y(1), \cdots, y(k-1)\}^{T} & \text { por: } \\
\hat{y}(k \mid k-1) & =E[y(k) \mid y(0), y(1), \cdots, y(k-1)] \\
& =E\left[y(k) \mid Y^{k-1}\right] \\
& =E\left[C_{d} x(k)+D_{d} u(k)+v(k) \mid Y^{k-1}\right] \\
& =C_{d} \hat{x}(k \mid k-1)
\end{aligned}
$$

Como pode ser observado através da equação (4.26), $\hat{y}(k \mid k-1)$ está relacionado com $\hat{x}(k \mid k-1)$ apenas através da matriz de observação $C_{d}$. 
As relações anteriores envolvendo a função de resposta ao impulso podem ser utilizadas para predizer o valor mais provável da resposta $y(k+n)$, dados a entrada $u(k)$ e a resposta $y(s)$ para instantes de tempo $s \leq k$. Da equação (4.20) verifica-se que o valor exato de $y(k+n)$ é dado por:

$$
\begin{aligned}
y(k+n) & =\sum_{j=0}^{\infty} h(j) u(k+n-j) \\
& =\sum_{j=0}^{n-1} h(j) u(k+n-j)+\sum_{j=n}^{\infty} h(j) u(k+n-j)
\end{aligned}
$$

A primeira soma da equação (4.27) tem média igual a zero e envolve apenas as entradas de $u(k+1)$ à $u(k+n)$, o que mostra ser independente dos acontecimentos até o instante de tempo $k$. Já a segunda soma é conhecida no tempo $k$ e representa a média condicional $\hat{y}(k+n \mid k)$ de $y(k+n)$, conhecidas as respostas $y(s)$, para instantes de tempo $s \leq k$. Assim o preditor de resposta $\hat{y}(k+n \mid k)$ é dado por:

$$
\hat{y}(k+n \mid k)=\sum_{j=n}^{\infty} h(j) u(k+n-j)
$$

A equação (4.28) pode ser reescrita da seguinte maneira:

$$
\begin{aligned}
\hat{y}(k+n \mid k)= & \sum_{j=n+1}^{\infty} h(j) u(k+n-j)+h(n) u(k) \\
& =\hat{y}(k+n \mid k-1)+h(n) u(k)
\end{aligned}
$$

e para $n=0$ tem-se:

$$
\hat{y}(k \mid k)=\hat{y}(k \mid k-1)+h(0) u(k) \quad \text { ou } \quad y(k)=\hat{y}(k \mid k-1)+B_{0} u(k)
$$

uma vez que $h(0)=B_{0}$ como mostrado em (4.23). 
Se a equação (4.28) é combinada com a equação de (4.24-a), e se $n \geq p$ o seguinte resultado pode ser obtido:

$$
\begin{aligned}
\hat{y}(k+n \mid k) & =\sum_{j=n}^{\infty}[h(j) u(k+n-j) \\
& =\sum_{j=n}^{\infty}\left(-\sum_{i=1}^{p} A_{i} h(j-i)+B_{j}\right) u(k+n-j) \\
& =-\sum_{i=1}^{p} A_{i}\left(\sum_{j=n}^{\infty} h(j-i) u(k+n-j)\right)+\sum_{j=n}^{\infty} B_{j} u(k+n-j)
\end{aligned}
$$

Da equação (4.24-b), sabe-se que $\left[B_{j}\right]=0$ para $j>p$ e como $j=n, \cdots, \infty$ e $n \geq p$ então somente $B_{n} \neq 0$, desta forma segue de (4.31) que:

$$
\begin{aligned}
& \hat{y}(k+n \mid k)=-\sum_{i=1}^{p} A_{i}\left(\sum_{j=n}^{\infty} h(j-i) u(k+n-j)\right)+B_{n} u(k) \\
& =-\sum_{i=1}^{p} A_{i} \hat{y}(k+n-i \mid k)+B_{n} u(k)
\end{aligned}
$$

Decompondo essa última equação para $j=n+1, \cdots, \infty$ e $j=n$, respectivamente temse:

$$
\begin{aligned}
\hat{y}(k+n \mid k)= & -\sum_{i=1}^{p} A_{i}\left(\sum_{j=n+1}^{\infty} h(j-i) u(k+n-j)\right)-\sum_{i=1}^{p} A_{i} h(n-i) u(k)+B_{n} u(k) \\
& =-\sum_{i=1}^{p} A_{i} \hat{y}(k+n-i \mid k-1)-\sum_{i=1}^{p} A_{i} h(n-i) u(k)+B_{n} u(k) \\
& =-\sum_{i=1}^{p} A_{i} \hat{y}(k+n-i \mid k-1)-\left(\sum_{i=1}^{p} A_{i} h(n-i)+B_{n}\right) u(k)
\end{aligned}
$$

Fazendo $j=n$ na equação (4.24-a) a equação acima pode ser reescrita da seguinte forma: 


$$
\hat{y}(k+n \mid k)=-\sum_{i=1}^{p} A_{i} \hat{y}(k+n-i \mid k-1)+h(n) u(k)
$$

uma vez, que $n \geq p$.

Baseando-se nessas relações, já é possível alcançar o objetivo proposto, isto é, formular a realização em espaço de estado, a qual será apresentada através do seguinte teorema.

\section{Teorema 4.2: Realização em espaço de estado do modelo ARMAV}

Uma realização em espaço de estado do modelo $\operatorname{ARMAV}(p, p)$ definido em (4.6), denominada de observabilidade canônica é dado por:

$$
\left\{\begin{array}{c}
x(k+1)=A_{d} x(k)+B_{d} u(k) \\
y(k)=C_{d} x(k)+D_{d} u(k)
\end{array}\right.
$$

onde as matrizes $A_{d}, B_{d}, C_{d}$ e $D_{d}$ são definidas da seguinte forma:

$$
\begin{gathered}
A_{d}=\left[\begin{array}{ccccccc}
0 & I & 0 & \cdots & 0 & 0 \\
0 & 0 & I & \cdots & 0 & 0 \\
0 & 0 & 0 & \cdots & 0 & 0 \\
\vdots & \vdots & \vdots & \vdots & \vdots & \vdots \\
0 & 0 & \cdots & \cdots & 0 & I \\
-A_{p} & -A_{p-1} & \cdots & \cdots & -A_{2} & -A_{1}
\end{array}\right] \\
B_{d}=\left[\begin{array}{cccccc}
I & 0 & 0 & \cdots & 0 & 0 \\
A_{1} & I & 0 & \cdots & 0 & 0 \\
A_{2} & A_{1} & I & \cdots & 0 & 0 \\
A_{3} & A_{2} & A_{1} & I & \vdots & \vdots \\
\vdots & \vdots & \vdots & \vdots & \ddots & \vdots \\
A_{p-1} & A_{p-2} & \cdots & \cdots & A_{1} & I
\end{array}\right]^{-1}\left[\begin{array}{c}
B_{1}-A_{1} B_{0} \\
B_{2}-A_{2} B_{0} \\
B_{3}-A_{3} B_{0} \\
B_{4}-A_{4} B_{0} \\
\vdots \\
B_{p}-A_{p} B_{0}
\end{array}\right]
\end{gathered}
$$




$$
C_{d}=\left[\begin{array}{lllll}
I & 0 & \cdots & 0 & 0
\end{array}\right] \quad D_{d}=B_{0}
$$

e o vetor de estado $x(k)$ é definido por

$$
x(k)=\left\{\begin{array}{c}
\hat{y}(k \mid k-1) \\
\hat{y}(k+1 \mid k-1) \\
\vdots \\
\vdots \\
\hat{y}(k+p-2 \mid k-1) \\
\hat{y}(k+p-1 \mid k-1)
\end{array}\right\}
$$

\section{Prova do Teorema 4.2:}

Através das equações dadas por (4.29) e (4.32) para $\hat{y}(k+n \mid k)$ com $n=1,2, \cdots, p-1$ e $n=p$, respectivamente, obtém-se as equações:

$$
\begin{aligned}
& \hat{y}(k+1 \mid k)=\hat{y}(k+1 \mid k-1)+h(1) u(k) \\
& \hat{y}(k+2 \mid k)=\hat{y}(k+2 \mid k-1)+h(2) u(k) \\
& \quad \vdots \\
& \hat{y}(k+p-1 \mid k)=\hat{y}(k+p-1 \mid k-1)+h(p-1) u(k) \\
& \hat{y}(k+p \mid k)=-A_{p} \hat{y}(k \mid k-1)-\cdots-A_{1} \hat{y}(k+p-1 \mid k-1)+h(p) u(k)
\end{aligned}
$$

Reescrevendo as equações acima na forma matricial tem-se:

$$
\left\{\begin{array}{c}
\hat{y}(k+1 \mid k) \\
\hat{y}(k+2 \mid k) \\
\vdots \\
\vdots \\
\hat{y}(k+p-1 \mid k) \\
\hat{y}(k+p \mid k)
\end{array}\right\}=\left[\begin{array}{cccccc}
0 & I & 0 & \cdots & 0 & 0 \\
0 & 0 & I & \cdots & 0 & 0 \\
0 & 0 & 0 & \cdots & 0 & 0 \\
\vdots & \vdots & \vdots & \vdots & \vdots & \vdots \\
0 & 0 & \cdots & \cdots & 0 & I \\
-A_{p} & -A_{p-1} & \cdots & \cdots & -A_{2} & -A_{1}
\end{array}\right]\left\{\begin{array}{c}
\hat{y}(k \mid k-1) \\
\hat{y}(k+1 \mid k-1) \\
\vdots \\
\vdots \\
\hat{y}(k+p-2 \mid k-1) \\
\hat{y}(k+p-1 \mid k-1)
\end{array}\right\}+
$$

onde 


$$
A_{d}=\left[\begin{array}{cccccc}
0 & I & 0 & \cdots & 0 & 0 \\
0 & 0 & I & \cdots & 0 & 0 \\
0 & 0 & 0 & \cdots & 0 & 0 \\
\vdots & \vdots & \vdots & \vdots & \vdots & \vdots \\
0 & 0 & \cdots & \cdots & 0 & I \\
-A_{p} & -A_{p-1} & \cdots & \cdots & -A_{2} & -A_{1}
\end{array}\right] \quad \text { e } \quad B_{d}=\left[\begin{array}{c}
h(1) \\
h(2) \\
\vdots \\
\vdots \\
h(p-1) \\
h(p)
\end{array}\right]
$$

Em notação simplificada a equação de estado é dada por:

$$
x(k+1)=A_{d} x(k)+B_{d} u(k)
$$

A matriz $B_{d}$ é constituída das funções de resposta ao impulso, e como já visto na equação (4.25), pode ser expressa em termos dos coeficientes matriciais do modelo ARMAV como em (4.34).

A equação de saída pode ser encontrada utilizando a equação (4.30), ou seja:

$$
\begin{aligned}
y(k)= & \hat{y}(k \mid k-1)+B_{0} u(k) \\
& =\left[\begin{array}{lllll}
I & 0 & \cdots & 0 & 0
\end{array}\right] x(k)+B_{0} u(k) \\
& =C_{d} x(k)+D_{d} u(k)
\end{aligned}
$$

onde $C_{d}$ é a matriz de influência de saída e $D_{d}$ é a matriz de transmissão direta. Com isso conclui-se a demonstração do Teorema 4.2.

Essa realização em espaço de estado é chamada de observabilidade canônica porque a matriz associada à matriz de observabilidade $Q o(p)$ é uma matriz identidade. A matriz de estado $A_{d}$ de dimensão $p m \times p m$, conhecida como matriz companheira é constituída das matrizes dos coeficientes auto-regressivos $A_{i}$ de dimensão $m \times m$, uma vez que $m$ saídas são observadas. A matriz de influência de entrada $B_{d}$ com dimensão $p m \times r$ consiste das $p$ funções de resposta ao impulso. Já a matriz de influência de saída $C_{d}$ cuja dimensão é $m \times p m$, assegura que apenas a resposta do sistema $y(k)$ é 
retornada, enquanto que a matriz de transmissão direta $D_{d}$, como já pôde ser visto, equivale ao coeficiente matricial de médias móveis $B_{0}$ de dimensão $m \times r$.

\subsection{DECOMPOSIÇÃO MODAL DA REALIZAÇÃO EM ESPAÇO DE ESTADO DO MODELO ARMAV}

Esta seção dedica-se a apresentar a decomposição modal da realização em espaço de estado do modelo ARMAV, de forma análoga àquela realizada na seção 3.4 do capítulo três.

Se a dimensão de estado $2 n$ dividido pelo número de saídas $m$ é igual a um valor inteiro $p$, e se o sistema é observável, então é possível representar vibrações livres por um polinômio matricial auto-regressivo $m$-dimensional de ordem $p$. De acordo com (4.11) os coeficientes matriciais auto-regressivos satisfazem a relação:

$$
C_{d} A_{d}^{p}+A_{1} C_{d} A_{d}^{p-1}+\cdots+A_{p-1} C_{d} A_{d}+A_{p} C_{d}=0
$$

Inserindo (3.14) em (4.36) obtém-se:

$$
C_{d} \Psi \lambda^{p} \Psi^{-1}+A_{1} C_{d} \Psi \lambda^{p-1} \Psi^{-1}+\cdots+A_{p-1} C_{d} \Psi \lambda \Psi^{-1}+A_{p} C_{d}=0
$$

e pós multiplicando a equação acima por $\Psi$ segue que:

$$
C_{d} \Psi \lambda^{p}+A_{1} C_{d} \Psi \lambda^{p-1}+\cdots+A_{p-1} C_{d} \Psi \lambda+A_{p} C_{d} \Psi=0
$$

Esta equação pode ser decomposta em $2 n$ equações separadamente. Cada uma delas tem a seguinte forma:

$$
\begin{aligned}
& C_{d} \Psi_{j} \lambda_{j}^{p}+A_{1} C_{d} \Psi_{j} \lambda_{j}^{p-1}+\cdots+A_{p-1} C_{d} \Psi_{j} \lambda_{j}+A_{p} C_{d} \Psi_{j}=0 \\
& \left(I \lambda_{j}^{p}+A_{1} \lambda_{j}^{p-1}+\cdots+A_{p-1} \lambda_{j}+A_{p}\right) C_{d} \Psi_{j}=0 \\
& \left(I \lambda_{j}^{p}+A_{1} \lambda_{j}^{p-1}+\cdots+A_{p-1} \lambda_{j}+A_{p}\right) \phi_{j}=0
\end{aligned}
$$


e pode ser vista como um problema de autovalor de ordem $p$ de polinômios matriciais auto-regressivo, considerando $\lambda_{j}$ como o autovalor e $\phi_{j}$ o modo de vibrar, com $j=1,2, \cdots, 2 n$.

Neste caso a decomposição modal dada pela equação (3.14) do sistema discreto será também a decomposição do polinômio matricial auto-regressivo associado.

Comparando o $j$-ésimo problema de autovalor em (4.37) com a estrutura da matriz companheira $A_{d}$ vista no teorema 4.2, é possível verificar que cada autovetor associado é dado por:

com $j=1, \cdots, 2 n$.

$$
\Psi_{j}=\left[\begin{array}{lllll}
\phi_{j} & \lambda_{j} \phi_{j} & \cdots & \lambda_{j}^{p-2} \phi_{j} & \lambda_{j}^{p-1} \phi_{j}
\end{array}\right]^{T}
$$




\section{Identificação de Sistemas Utilizando o Modelo ARMAV e ARV}

\subsection{PRELIMINARES}

A identificação estrutural de um sistema através de modelos paramétricos no domínio do tempo, tem por objetivo ajustar um modelo discreto de ordem finita que represente a dinâmica do sistema de forma otimizada. Neste trabalho serão utilizados na identificação de sistemas os modelos paramétricos ARMAV e ARV. Estes modelos, fundamentados na teoria de séries temporais, caracterizam-se através de uma relação funcional entre a entrada e saída do sistema.

A finalidade deste capítulo é apresentar técnicas de estimação dos coeficientes dos modelos paramétricos ARMAV e ARV. Uma vez estimados os coeficientes autoregressivos, os quais estão relacionados com o comportamento dinâmico do sistema, torna-se possível extrair os parâmetros modais do sistema físico.

No caso do modelo ARMAV, o sistema estrutural é identificado a partir dos dados de excitação e resposta, ou seja, através da AMT. Já a identificação com base no modelo ARV é realizada a partir da AMO, onde apenas as respostas do sistema são observadas. 
Além disso, este capítulo também aborda outras duas etapas importantes no processo de identificação: a determinação da ordem do modelo e a sua validação.

\subsection{MÉTODOS DE ESTIMAÇÃO DOS PARÂMETROS DOS MODELOS ARMAV E ARV}

Os métodos apresentados nesta seção para encontrar uma estimativa para os coeficientes dos modelos são baseados na aproximação dos mínimos quadrados (LS) e mínimos quadrados modificado, sendo esta última aproximação utilizada somente para o caso do modelo ARV.

\subsubsection{MÉTODO DOS MÍNIMOS QUADRADOS - MODELO ARMAV}

Como já visto no capítulo 4, o comportamento dinâmico de um sistema vibratório linear discreto e invariante no tempo pode ser descrito através de um modelo paramétrico $\operatorname{ARMAV}(p, p)$ representado através da equação de diferenças matricial:

$$
y(k)=-\sum_{i=1}^{p} A_{i} y(k-i)+\sum_{i=0}^{p} B_{i} u(k-i)
$$

onde $y(k)$ é o vetor de saídas observadas e $u(k)$ é o vetor de entradas, considerado como sendo um ruído branco gaussiano.

Por conveniência, neste trabalho a ordem da parcela auto-regressiva do modelo ARMAV é considerada coincidente com a ordem da parcela de média móvel. Como em situações reais, as medidas de respostas sempre são contaminadas por ruídos, o modelo $\operatorname{ARMAV}(p, p)$ adequado para obter boas estimativas dos parâmetros modais é, em geral, descrito por um modelo sobre-determinado onde a ordem $p$ deve ser definida de maneira que $p \geq \frac{2 n}{m}$ (ANDERSEN, 1997). 
Visto que o vetor de entradas do sistema $u(k)$ corresponde a um ruído branco de média zero, vale lembrar que as seguintes propriedades são válidas:

$$
\begin{aligned}
& \mathrm{E}[u(k)]=0 \\
& \mathrm{E}\left[u(k) u^{T}(k+j)\right]=\Delta \delta(j), \quad j=0,1,2, \cdots
\end{aligned}
$$

onde a primeira diz que a média do ruído branco é nula e a segunda representa a função de correlação, que mede o grau de dependência linear existente entre as amostras do ruído. Isto equivale a dizer que a densidade espectral de potência, ou seja, a transformada de Fourier da função correlação, é constante para todas as freqüências.

Para representar uma situação mais próxima do real, assume-se que as medidas de respostas $y(k)$ são corrompidas por um ruído branco $v(k)$, ou seja, $\hat{y}(k)=y(k)+v(k)$.

Desta forma, substituindo $y(k)=\hat{y}(k)-v(k)$ na equação (5.1) expandida obtém-se:

$$
\hat{y}(k)=-A_{1} \hat{y}(k-1)-\cdots-A_{p} \hat{y}(k-p)+B_{0} u(k-0)+B_{1} u(k-1)+\cdots+B_{p} u(k-p)+\varepsilon(k)
$$

onde o erro é definido por: $\varepsilon(k)=v(k)+A_{1} v(k-1)+A_{2} v(k-2)+\cdots+A_{\mathrm{p}} v(k-p)$.

Coletando informações de entradas e saídas medidas em instantes de tempo $k=p+1, p+2, \cdots, p+L$, onde $L \leq L_{\max }$ com $L_{\max }=l-p$, onde $l$ representa $\mathrm{o}$ tamanho total da amostra, e reescrevendo a equação (5.2) na forma matricial em todos os instantes de tempo $k$ tem-se:

$$
\begin{aligned}
{\left[\begin{array}{lllll}
\hat{y}(p+1) & \cdots & \hat{y}(p+\mathrm{L})
\end{array}\right]=} & {\left[\begin{array}{llllll}
-\hat{y}(p) & -\hat{y}(p+1) & \cdots & -\hat{y}(p+L-1) \\
\vdots & \vdots & \vdots & \vdots \\
-\hat{y}(1) & -\hat{y}(2) & \cdots & -\hat{y}(L) \\
u(p+1) & u(p+2) & \cdots & u(p+L) \\
\vdots & \vdots & \vdots & \vdots \\
u(1) & u(2) & \cdots & u(L)
\end{array}\right]+} \\
& +\left[\begin{array}{llllll}
\varepsilon(p+1) & \cdots & \varepsilon(p+L)
\end{array}\right]
\end{aligned}
$$


$\mathrm{Na}$ forma simplificada pode ser reescrita por:

$$
\hat{Y}=\theta \hat{S}+\hat{E}
$$

onde as matrizes $\hat{Y}, \theta, \hat{S}$ e $\hat{E}$ possuem dimensões $m \times L, \quad m \times(m p+r p+r)$, $(m p+r p+r) \times L$ e $m \times L$, respectivamente.

Desta maneira, os parâmetros do modelo ARMAV, que compõem a matriz $\theta$ podem ser determinados através da solução do sistema linear representado pela equação (5.3), utilizando a aproximação dos mínimos quadrados, para $L \geq(m p+r p+r)$.

Assim a matriz $\theta$ pode ser estimada minimizando a seguinte função critério escalar:

$$
\begin{aligned}
J(\theta) & =\frac{1}{L} \sum_{k=p+1}^{p+L} \sum_{i=1}^{m} \varepsilon_{i}^{2}(k)=\frac{1}{L} \sum_{k=p+1}^{p+L} \varepsilon^{T}(k) \varepsilon(k)= \\
& =\frac{1}{L} \operatorname{tr}\left(\hat{E} \hat{E}^{T}\right)=\frac{1}{L} \operatorname{tr}\left[(\hat{Y}-\theta \hat{S})(\hat{Y}-\theta \hat{S})^{T}\right]
\end{aligned}
$$

que descreve a soma dos erros quadráticos, onde $\operatorname{tr}($ ) é o operador traço.

Baseando-se em $L$ amostras, $\theta$ é então obtido como ponto de mínimo global da função critério (5.4), isto é:

$$
\theta=\arg \min _{\theta} J(\theta)
$$

Em (5.5) arg min deve ser lido como argumento que minimiza a função critério $J(\theta)$, também conhecida como função perda.

Expandindo a equação (5.4) que representa a função critério tem-se:

$$
J(\theta)=\frac{1}{L} \operatorname{tr}\left(\hat{Y} \hat{Y}^{T}-\hat{Y} \hat{S}^{T} \theta^{T}-\theta \hat{S} \hat{Y}^{T}+\theta \hat{S} \hat{S}^{T} \theta^{T}\right)
$$


Derivando $J(\theta)$ em relação à $\theta$ e utilizando as seguintes propriedades de derivada matricial (LEE e CHEN, 1989):

$$
\begin{aligned}
& \frac{\partial\left(\operatorname{tr}\left(A B A^{T}\right)\right)}{\partial A}=2 A B, \text { quando } B \text { é simétrica } \\
& \frac{\partial(\operatorname{tr}(A B))}{\partial A}=B^{T}
\end{aligned}
$$

segue que:

$$
\frac{\partial J(\theta)}{\partial \theta}=0-\hat{Y} \hat{S}^{T}-\hat{Y} \hat{S}^{T}+2 \theta \hat{S} \hat{S}^{T}
$$

Igualando a zero para $\theta=\hat{\theta}$, onde $\hat{\theta}$ é a estimativa dos mínimos quadrados, tem-se:

$$
\left.\frac{\partial J(\theta)}{\partial \theta}\right|_{\theta=\hat{\theta}}=0 \Rightarrow \hat{\theta}\left[\hat{S} \hat{S}^{T}\right]=\hat{Y} \hat{S}^{T}
$$

cujo sistema acima apresenta a forma de equações normais (LJUNG, 1987).

Considerando $\hat{S} \hat{S}^{T}$ inversível, obtém-se uma estimativa única de $\theta$ dada por:

$$
\hat{\theta}=\hat{Y} \hat{S}^{+}
$$

onde $\hat{S}^{+}=\hat{S}^{T}\left(\hat{S} \hat{S}^{T}\right)^{-1}$ é denominada de pseudo-inversa (Moore-Penrose) de $\hat{S}$.

\subsubsection{MÉTODO DOS MÍNIMOS QUADRADOS - MODELO ARV}

O modelo auto-regressivo vetorial ARV constitui uma das ramificações da classe do modelo ARMAV. A utilização do modelo ARV na identificação de sistemas fundamenta-se no ajuste de um modelo apenas aos dados de medidas de respostas $y(k)$. A vantagem de utilizar o modelo ARV quando a entrada não é medida, é que os 
coeficientes auto-regressivos que determinam a dinâmica do sistema, são estimados através de uma forma linear, enquanto que no modelo ARMAV os coeficientes devem ser estimados a partir de uma aproximação dos mínimos quadrados em dois ou mais estágios ou através de uma aproximação não linear. Além disso, estipulada uma ordem suficientemente grande, o modelo ARV torna-se rico o bastante para representar uma grande faixa de processos (XIA HE e DE ROECK, 1997).

Considerando o modelo ARMAV dado por (5.1) e removendo a parcela de média móvel obtém-se o modelo auto-regressivo vetorial $\operatorname{ARV}(p)$, de ordem $p$, representado pela equação:

$$
y(k)=-A_{1} y(k-1)-A_{2} y(k-2)-\cdots-A_{P} y(k-p)+u(k)
$$

onde $y(k)$ é a medida de resposta observada, $A_{i}$ são os coeficientes matriciais autoregressivos a serem determinados, para $i=1, \cdots, p$, e $u(k)$ corresponde a excitação do sistema, desconhecida no sentido determinístico e assumida como um processo de ruído branco.

O método considerado aqui para estimar os coeficientes auto-regressivos, consiste em extrair o máximo de informação dos dados, excluindo o processo de ruído branco $u(k)$ do modelo, que é não correlacionado com os dados de resposta em instantes de tempo passado. Isto pode ser escrito em termos do operador esperança $E[$ ], onde:

$$
\mathrm{E}\left[u(k) \cdot y^{T}(k-j)\right]=\mathrm{E}[u(k)] \mathrm{E}\left[y^{T}(k-j)\right]=0
$$

para $\forall j \neq 0$ inteiro. A primeira igualdade diz que $u(k)$ e $y^{T}(k-j)$ são nãocorrelacionados e a segunda igualdade segue da propriedade de que a média de um ruído branco é igual a zero.

A derivação do método parte da imposição (5.9) aplicada ao modelo ARV representado pela equação (5.8). Desta forma, pós-multiplicando ambos os lados da equação (5.8) por $y^{T}(k-j)$ e tomando a esperança obtém-se: 


$$
\begin{aligned}
\mathrm{E}\left[y(k) y^{T}(k-j)\right]= & -A_{1} \mathrm{E}\left[y(k-1) y^{T}(k-j)\right]-\cdots-A_{p} \mathrm{E}\left[y(k-p) y^{T}(k-j)\right]+ \\
& +\mathrm{E}\left[u(k) y^{T}(k-j)\right]
\end{aligned}
$$

Aplicando a propriedade (5.9), a equação (5.10) pode ser reescrita com eliminação de seu último termo:

$$
\mathrm{E}\left[y(k) y^{T}(k-j)\right]=-A_{1} \mathrm{E}\left[y(k-1) y^{T}(k-j)\right]-\cdots-A_{p} \mathrm{E}\left[y(k-p) y^{T}(k-j)\right]
$$

Utilizando as propriedades válidas para um processo estacionário (1.2), encontradas no Apêndice, a equação (5.11) pode ser escrita em termos das funções de correlação dos dados de saída do sistema:

$$
R(-j)=-A_{1} R(1-j)-A_{2} R(2-j)-\cdots-A_{p} R(p-j)
$$

Representando-a em uma forma compacta tem-se:

$$
R(-j)=-\sum_{i=1}^{p} A_{i} R(i-j)
$$

ou equivalentemente:

$$
R^{T}(j)=-\sum_{i=1}^{p} A_{i} R^{T}(j-i)
$$

Por conveniência, aplicando a operação transposta de ambos os lados da equação (5.13) e expandindo-a para valores de $j \neq 0$ com $j=1, \cdots, \widetilde{L}$, tem-se o seguinte sistema:

$$
\left[\begin{array}{ccccc}
R(0) & R(-1) & R(-2) & \cdots & R(1-p) \\
R(1) & R(0) & R(-1) & \cdots & R(2-p) \\
R(2) & R(1) & R(0) & \cdots & R(3-p) \\
\vdots & \vdots & \vdots & \vdots & \vdots \\
R(\widetilde{L}-1) & R(\widetilde{L}-2) & R(\widetilde{L}-3) & \cdots & R(\widetilde{L}-p)
\end{array}\right]\left[\begin{array}{c}
A_{1}{ }^{T} \\
A_{2}{ }^{T} \\
A_{3}{ }^{T} \\
\vdots \\
A_{p}{ }^{T}
\end{array}\right]=\left[\begin{array}{c}
-R(1) \\
-R(2) \\
-R(3) \\
\vdots \\
-R(\widetilde{L})
\end{array}\right]
$$


Representando-o numa forma simplificada tem-se:

$$
\widetilde{S} \theta=\widetilde{U}
$$

onde as matrizes $\widetilde{S}, \theta$ e $\widetilde{U}$ apresentam dimensões $m \widetilde{L} \times m p, m p \times m$ e $m \widetilde{L} \times m$, respectivamente. A solução do sistema (5.14) é determinada através da aproximação dos mínimos quadrados tradicional, para $\widetilde{L} \gg p$. Sendo assim, a estimativa de $\theta$ é dada por:

$$
\widetilde{\theta}=\widetilde{S}^{+} \widetilde{U}
$$

onde $\widetilde{S}^{+}=\left(\widetilde{S}^{T} \widetilde{S}\right)^{-1} \widetilde{S}^{T}$ é chamada de pseudo-inversa de $\widetilde{S}$.

Para determinar $\widetilde{\theta}$, ou seja, os coeficientes matriciais do modelo $\operatorname{ARV}(p)$, tornase necessário estimar as matrizes de correlação das medidas de respostas, que compõem as matrizes $\widetilde{S}$ e $\widetilde{U}$. Para tanto, a Hipótese Ergódica deve ser considerada. Esta hipótese diz que as propriedades estatísticas, tais como a média e as funções de correlação de um processo estacionário, podem ser calculadas através de um único sinal, amostrado em um intervalo de tempo suficientemente grande, de forma a conter todas as informações estatísticas do processo.

$\mathrm{Na}$ prática, os processos aleatórios não são verdadeiramente estacionários, no entanto longas observações do processo exibem características que permitem que eles sejam tratados como estacionários. Do ponto de vista da análise dinâmica, o regime transiente é descrito como sendo um processo não estacionário com o seu início e fím claramente definidos em um curto intervalo de tempo (BENDAT e PIERSOL, 1993). No entanto, a maioria dos resultados na identificação de sistemas dinâmicos utiliza resultados baseados na resposta do regime permanente que é considerado um processo estacionário observado em um longo período de tempo, satisfazendo portanto, as propriedades ergódicas (BROWN e HWANG, 1992)

De acordo com a definição de função de correlação dada no Apêndice A por (1.1) e supondo válida a Hipótese Ergódica, a função de correlação dos dados de saída $y(k)$ é definida por: 


$$
R(j)=E\left[y(k) y^{T}(k+j)\right]=\lim _{l \rightarrow \infty} \frac{1}{l} \sum_{k=0}^{l-1} y(k) y^{T}(k+j)
$$

onde o vetor de saídas $y(k)$ é avaliado nos instantes $k=0,1, \cdots, l-1$.

Em situações práticas, a função de correlação dada por (5.15) não é conhecida, uma vez que somente um número finito de dados pode ser medido. Assim sendo, a estimativa para essa função é dada através de um número finito de dados avaliados a partir do truncamento do limite acima. Neste trabalho, será utilizada a estimativa “biased” da função de correlação (MARPLE, 1988):

$$
R(j)= \begin{cases}\frac{1}{l} \sum_{k=0}^{l-j-1} y(k) y^{T}(k+j) & \text { para } j \geq 0 \\ R^{T}(-j) & \text { para } j<0\end{cases}
$$

\subsubsection{MÉTODO DOS MÍNIMOS QUADRADOS MODIFICADO - MODELO ARV}

A modificação do método dos mínimos quadrados proposta por HUANG (2001), baseia-se na relação de equivalência teórica existente entre a matriz de correlação $R(j)$ das respostas de um sistema linear, sujeito à excitação ruído branco $u(k)$, e a resposta livre do sistema estrutural considerado. HUANG e YEH (1999) mostraram que para entradas do tipo ruído branco Gaussiano, cada linha da matriz de correlação de respostas $R(j)$, do tipo deslocamento ou velocidade é idêntica à resposta de vibração livre do sistema sob certas condições iniciais. Porém, para respostas tomadas como aceleração, essa relação de equivalência é válida somente se a função de correlação de resposta $R(j)$ é avaliada apenas para valores de $j>0$.

Sem perda de generalidade, um sistema de 2 graus de liberdade com amortecimento proporcional será utilizado para discutir este relacionamento. As equações do movimento que representam o sistema, em termos dos deslocamentos relativos à base: $z_{1}(t)=w_{1}(t)-w_{b}(t)$ e $z_{2}(t)=w_{2}(t)-w_{b}(t)$, onde $w_{b}(t)$ representa 0 
deslocamento da base, e $w_{1}(t)$ e $w_{2}(t)$ os deslocamentos das massas estruturais, podem ser descritas através da seguinte forma matricial:

$$
\left[\begin{array}{cc}
m_{1} & 0 \\
0 & m_{2}
\end{array}\right]\left\{\begin{array}{l}
\ddot{z}_{1}(t) \\
\ddot{z}_{2}(t)
\end{array}\right\}+\left[\begin{array}{cc}
\left(c_{1}+c_{2}\right) & -c_{2} \\
-c_{2} & c_{2}
\end{array}\right]\left\{\begin{array}{l}
\dot{z}_{1}(t) \\
\dot{z}_{2}(t)
\end{array}\right\}+\left[\begin{array}{cc}
\left(k_{1}+k_{2}\right) & -k_{2} \\
-k_{2} & k_{2}
\end{array}\right]\left\{\begin{array}{l}
z_{1}(t) \\
z_{2}(t)
\end{array}\right\}=\left\{\begin{array}{l}
-m_{1} \ddot{w}_{b}(t) \\
-m_{2} \ddot{w}_{b}(t)
\end{array}\right\}
$$

Assumindo que a aceleração da base $\ddot{w}_{b}(t)$ é um ruído branco de media zero e autospectro unitário com distribuição gaussiana, e baseando-se na definição da função de correlação, os seguintes resultados podem ser obtidos através de algumas operações matemáticas fundamentais (HUANG, TSAI e YEH, 1998* (apud HUANG, C. S., 2001)) para as:

- funções de auto-correlação e correlação cruzada, quando respostas de deslocamento ou velocidade são consideradas:

$$
\begin{gathered}
\left\{\begin{array}{l}
R_{z_{z_{1}}}(j) \\
R_{z_{1} z_{2}}(j)
\end{array}\right\}=S^{d} \sum_{i=1}^{2} e^{-\zeta_{i} \omega_{i} j \Delta t}\left(A_{i}^{d} \cos \left(\omega_{d i} j \Delta t\right)+B_{i}^{d} \operatorname{sen}\left(\omega_{d i} j \Delta t\right)\right) \phi_{i} \\
\left\{\begin{array}{l}
R_{\dot{z}_{1} \dot{z}_{1}}(j) \\
R_{\dot{z}_{1} \dot{z}_{2}}
\end{array}\right\}=S^{v} \sum_{i=1}^{2} e^{-\zeta_{i} \omega_{i} j \Delta t}\left(A_{i}^{v} \cos \left(\omega_{d i} j \Delta t\right)+B_{i}^{v} \operatorname{sen}\left(\omega_{d i} j \Delta t\right)\right) \phi_{i}
\end{gathered}
$$

- funções de auto-correlação e correlação cruzada, quando respostas de aceleração são analisadas:

$$
\begin{aligned}
\left\{\begin{array}{l}
R_{\ddot{z}_{1_{1}} \ddot{1}_{1}}(j) \\
R_{\ddot{z}_{1} \ddot{z}_{2}}(j)
\end{array}\right\} & =S^{a} \sum_{i=1}^{2} e^{-\zeta_{i} \omega_{i} j \Delta t}\left(A_{i}^{a} \cos \left(\omega_{d i} j \Delta t\right)+B_{i}^{a} \operatorname{sen}\left(\omega_{d i} j \Delta t\right)\right) \phi_{i}+ \\
& +S^{a} \delta(j \Delta t) \sum_{i=1}^{2} C_{i}^{a} \phi_{i}
\end{aligned}
$$

Os parâmetros $\omega_{i}, \zeta_{i}, \omega_{d i}$ e $\phi_{i}$ para $i=1,2$ representam respectivamente as freqüências naturais, fatores de amortecimento, freqüências amortecidas e modos de vibrar do sistema dado por (5.17). $A_{i}^{d}, B_{i}^{d}, S^{d}, A_{i}^{v}, B_{i}^{v}, S^{v}, A_{i}^{a}, B_{i}^{a}$ e $S^{a}$ são constantes

\footnotetext{
${ }^{*}$ HUANG, C. S., TSAI, I. C. AND YEH, C. H. (1998). Application of random decrement technique to identify the characteristics of a building in time domain from ambient vibration measurement (in chinese). Journal of the Chinese Institute of Civil and Hydraulic Engineering, v.10 p.537-547. apud HUANG, C. S. (2001). Structural identification from ambient vibration measurement using the multivariate AR model. Journal of Sound and Vibration, v.241, n.3, p.337-359, July.
} 
que dependem das propriedades do sistema, e suas expressões são encontradas em (HUANG, TSAI e YEH, 1998* (apud HUANG, C. S., 2001)).

As equações (5.18) e (5.19) são equivalentes à resposta de vibração livre do sistema estrutural descrito em (5.17) com certas condições iniciais. Isto se verifica uma vez que as funções de correlação de respostas de deslocamento ou velocidade são descritas como soma de senos amortecidos, onde cada "decaimento" senoidal apresenta uma freqüência natural amortecida e um fator de amortecimento, idênticos aos do modo estrutural correspondente.

Porém, quando a resposta do sistema é tomada como aceleração, a equivalência permanece válida somente para valores de $j>0$, visto que para esses valores a equação (5.20), reduz-se a:

$$
\left\{\begin{array}{l}
R_{\ddot{z}_{\ddot{z}_{1}}}(j) \\
R_{\ddot{z}_{1} \ddot{z}_{2}}(j)
\end{array}\right\}=S^{a} \sum_{i=1}^{2} e^{-\zeta_{i} \omega_{i} j \Delta t}\left(A_{i}^{a} \cos \left(\omega_{d i} j \Delta t\right)+B_{i}^{a} \operatorname{sen}\left(\omega_{d i} j \Delta t\right)\right) \phi_{i}
$$

uma vez que, $\delta(j \Delta t)=0$ para $j \neq 0$.

Sendo assim, para o caso da resposta de aceleração, a equação (5.13), que representa o modelo ARV descrito através da função de correlação, torna-se equivalente à expressão da resposta livre do sistema contínuo, somente se valores adequados de $j$ são utilizados.

Neste trabalho será considerada apenas resposta do tipo aceleração.

Além disso, em aplicações práticas, as medidas de respostas sempre são contaminadas por ruídos. Se o ruído presente nos dados é similar ao ruído branco então:

$$
\hat{R}(j)=R(j)+\bar{\Delta} \bar{\delta}(j)
$$

onde $\hat{R}(j)$ e $R(j)$ são matrizes de função de correlação das medidas de respostas com ruído e livre de ruído, respectivamente, enquanto que $\bar{\Delta}$ é a matriz de covariância do ruído e $\bar{\delta}(j)=1$ se $j=0 ; \bar{\delta}(j)=0$ se $j \neq 0$. Por mais essa razão, nota-se que a existência de equivalência é destruída quando $j=0$.

Conseqüentemente, é proposta uma modificação em cada elemento que compõe as matrizes $\tilde{S}$ e $\widetilde{U}$ do sistema (5.14) de forma a manter a relação de equivalência. As 
matrizes $\widetilde{S}$ e $\widetilde{U}$ modificadas são denotadas por $\widetilde{S}_{\text {modif }}$ e $\widetilde{U}_{\text {modif }}$, respectivamente e descritas por:

$$
\begin{gathered}
\widetilde{S}_{\text {modif }}=\left[\begin{array}{ccccc}
R\left(0+k_{i}\right) & R\left(-1+k_{i}\right) & R\left(-2+k_{i}\right) & \cdots & R\left(1-p+k_{i}\right) \\
R\left(1+k_{i}\right) & R\left(0+k_{i}\right) & R\left(-1+k_{i}\right) & \cdots & R\left(2-p+k_{i}\right) \\
R\left(2+k_{i}\right) & R\left(1+k_{i}\right) & R\left(0+k_{i}\right) & \cdots & R\left(3-p+k_{i}\right) \\
\vdots & \vdots & \vdots & \vdots & \vdots \\
R\left(\widetilde{L}-1+k_{i}\right) & R\left(\widetilde{L}-2+k_{i}\right) & R\left(\widetilde{L}-3+k_{i}\right) & \cdots & R\left(\widetilde{L}-p+k_{i}\right)
\end{array}\right] \\
\widetilde{U}_{\text {modif }}=\left[\begin{array}{lllll}
-R^{T}\left(1+k_{i}\right) & -R^{T}\left(2+k_{i}\right) & -R^{T}\left(3+k_{i}\right) & \cdots & -R^{T}\left(\widetilde{L}+k_{i}\right)
\end{array}\right]^{T}
\end{gathered}
$$

onde $k_{i}>p-1$, para que nenhum elemento da matriz $\widetilde{U}_{\text {modif }}$ tenha índice de defasagem temporal "lag" zero. Com base em experiências numéricas, sugere-se que $k_{i}$ seja escolhido de tal forma que $k_{i} \in(p+4, p+14)$.

Obviamente, para $k_{i}=0, \widetilde{S}_{\text {modif }}=\tilde{S}$ e $\widetilde{U}_{\text {modif }}=\tilde{U}$.

Com essa pequena modificação, fundamentada na relação de equivalência entre a matriz de correlação das respostas de um sistema linear e a resposta livre do sistema de vibração, a versão modificada pode alcançar uma eficiência considerável na determinação das características dinâmicas do sistema, em relação ao método tradicional.

\subsection{PROCEDIMENTOS DE DETERMINAÇÃO DA ORDEM DO MODELO}

O problema da determinação da ordem do modelo é considerado um dos passos mais importantes no processo de identificação de sistemas. Embora as estruturas mecânicas possuam infinitos graus de liberdade, consequentemente, infinitas freqüências naturais, fatores de amortecimento e modos de vibrar, a modelagem de 
sistemas físicos e os ensaios experimentais requerem apenas um número finito de modos contidos e uma determinada faixa operacional de freqüência.

A dificuldade em determinar a ordem do modelo está associada aos erros devidos à presença de ruído no sinal. Dentre as principais fontes de ruído no processo de identificação estão à imprecisão na modelagem, imperfeições nos instrumentos de medidas e erros computacionais devidos a truncamentos.

Visto que a ordem do modelo está associada à capacidade do mesmo em evidenciar características relevantes ao comportamento dinâmico do sistema, cuidados devem ser tomados quanto à sua determinação.

A utilização de modelos sub-determinados, isto é, modelos que apresentam ordem menor do que a ordem exata na identificação dos parâmetros modais, resulta na estimação pobre de componentes de alta freqüência do sinal e razoável estimação de componentes de baixa freqüência.

Por outro lado, a estimação realizada a partir de modelos sobre-determinados é uma prática comum no processo de identificação, uma vez que possibilita estimar de forma mais precisa os parâmetros modais numa faixa de freqüência considerável. No entanto, modelos sobre-determinados contém informações relacionadas aos modos do sistema e relativas aos modos computacionais que aparecem no modelo para compensar o efeito de ruídos ou outras irregularidades de medidas. As desvantagens do uso de modelos sobre-determinados estão relacionadas a problemas de mal condicionamento matricial e às dificuldades em realizar a distinção entre os modos estruturais, que representam o sistema dinâmico, e os modos computacionais.

Dentre os diferentes critérios de determinação da ordem do modelo encontrados na literatura destacam-se: o critério da variável instrumental (DUONG e LANDAU, 1996) decomposição em valor singular (SVD) (ANDERSEN, 1997), e aqueles baseados nas propriedades estatísticas dos erros de predição residual tais como: o critério do erro preditor final (FPE) fundamentado na variância do erro preditor (AKAIKE, 1969), o critério de informação de Akaike (AIC) que deriva de informação de conceitos teóricos (AKAIKE, 1974), o critério de informação Bayesiana (BIC) (WEI, 1990) e o critério do comprimento de descrição mínima (MDL) (RISSANEN,1983).

A questão de encontrar a ordem que melhor representa um modelo tem sido alvo de muitas pesquisas e muitas técnicas têm sido propostas com base nos critérios citados, como por exemplo, aquelas encontradas nos trabalhos de (VAN DEN BOOM e VAN 
DEN ENDEN, 1974; PUKKILA e KRISHNAIAH, 1988; LIANG, WIEKES e CADZOW, 1993; SMAIL e THOMAS, 1999; LARBI e LARDIES, 2000).

Dentre os critérios estatísticos citados, neste trabalho será utilizado apenas o critério BIC que é uma evolução do critério FPE. Este critério foi escolhido, devido ao seu melhor desempenho na determinação da ordem dos modelos estudados neste trabalho, comparado com os resultados obtidos a partir dos critérios FPE e AIC. O critério BIC, que utiliza conceitos estatísticos de Bayes (SHWARZ,1978), tem sido largamente empregado em situações práticas devido à sua robustez na determinação da ordem de modelos paramétricos. Sua formulação é baseada na análise dos erros residuais de modelos de diferentes ordens. O critério BIC é definido pela seguinte relação (PAPAKOS e FASSOIS, 2003):

$$
B I C(p)=\ln (\operatorname{det}(\Omega))+\rho \frac{\ln L}{L}
$$

onde $\rho$ representa o número de parâmetros ajustáveis do modelo estimado, $L$ o tamanho da amostra, $\ln$ o logaritmo natural e $\Omega$ a matriz de covariância do erro residual que pode ser estimada por:

$$
\Omega=\frac{1}{L} \hat{E} \hat{E}^{T}
$$

A ordem $p$ ótima do modelo, dada por este critério, é aquela que minimiza a função $B I C(p)$.

Além dos critérios apresentados, o diagrama de estabilidade também tem sido amplamente utilizado como auxílio na determinação da ordem do modelo. O diagrama de estabilidade é uma poderosa ferramenta visual que apresenta informação sobre a estabilidade dos parâmetros modais, geralmente as freqüências, identificadas a partir de várias estimativas. Com base no diagrama, um modelo de ordem mais adequada para representar o sistema é encontrado tão logo os parâmetros estimados se estabilizam. Isso se deve ao fato de que os modos estruturais tendem a se estabilizarem enquanto os modos computacionais variam acentuadamente de uma estimativa para outra, uma vez que o ruído não é correlacionado com os dados de entrada e saída do sistema.

Neste trabalho o critério BIC alcançou resultados satisfatórios nas identificações realizadas com base no modelo ARMAV. No entanto, para o caso da identificação a partir do modelo ARV foi proposta uma nova técnica para solucionar o problema de 
determinação da ordem do modelo. Para tanto, foi desenvolvida uma metodologia no processo de identificação utilizando o modelo $\operatorname{ARV}(p)$, com o auxílio do diagrama de estabilidade aliado a um critério analítico baseado no método de regressão linear. Essa metodologia, que será apresentada no capítulo seis, além de solucionar o problema de encontrar a ordem que melhor representa o sistema, contribuiu também para automatizar o processo de identificação.

\subsection{VALIDAÇÃO DO MODELO}

A validação do modelo é o estágio final do procedimento de identificação de sistemas. É sempre importante verificar se o modelo é um bom ajuste dos dados pelos quais ele foi estimado. Neste contexto, a comparação entre a saída medida e a saída simulada do modelo pode ser utilizada como um critério simples de validação.

No entanto é recomendável que os testes estatísticos, baseados na análise do sinal caracterizado pela diferença entre os valores estimados na série e valores medidos, denominado de erro residual, sejam utilizados como indicadores numéricos da validação do modelo. Esta análise é fundamentada na hipótese de que a entrada do sistema utilizada nos modelos ARMAV e ARV seja caracterizada por um ruído branco. Portanto é esperado que o erro residual apresente características de um ruído branco. O teste clássico de verificação do comportamento do erro residual baseia-se na análise do gráfico da autocorrelação do mesmo (LJUNG, 1987 e SÖDERSTRÖM e STOICA, 1989). Uma vez que o processo de excitação é admitido como um ruído branco; é esperado que a série temporal obtida pela diferença entre os valores estimados e valores medidos seja não correlacionada para atrasos superiores a zero, ou seja, a função de autocorrelação do erro residual permanece em sua maior parte, dentro de um intervalo de confiança, exceto no "lag" zero.

Além de verificar se o erro residual é um ruído branco, é necessário também averiguar se o mesmo assume uma distribuição gaussiana (BODEUX e GOLINVAL, 2003). Se a distribuição estatística do erro residual concorda com a distribuição estatística da excitação, então se pode concluir que o sistema dinâmico é de fato bem representado pelo modelo. Qualquer tendência diferente nas características estatísticas 
daquelas originalmente adotadas é uma indicação de que o modelo ou o ruído foi assumido de maneira incorreta ou que os parâmetros foram estimados incorretamente.

Exemplos de aplicação destes critérios de validação, utilizando o modelo ARMAV, serão mostrados nos capítulos seis e sete.

Os algoritmos computacionais das técnicas de identificação discutidas neste capítulo serão apresentados no capítulo seguinte. A aplicação desses algoritmos a dados provenientes de simulações numéricas será realizada no capítulo seis, e a dados obtidos a partir de um modelo experimental apresentada no capítulo sete. 


\section{Aspectos Computacionais Simulações Numéricas}

\subsection{PRELIMINARES}

Neste capítulo são apresentados os algoritmos numéricos de identificação, através de fluxogramas, que mostram as principais etapas da implementação dos métodos de identificação descritos no capítulo cinco.

Estes algoritmos serão aplicados aos dados provenientes de simulações numéricas com o objetivo de ilustrar algumas características importantes dos métodos estudados, bem como verificar a eficiência das técnicas de identificação utilizando o modelo ARMAV e ARV. Para tanto, realizou-se um estudo onde foram considerados sistemas com quatro e cinco graus de liberdade e com algumas características especiais tais como: freqüências distantes e grandes amortecimentos, bem como freqüências próximas e amortecimentos baixos. Além disso, as simulações numéricas foram realizadas considerando também os dados de respostas corrompidos por ruído e tomando apenas algumas medidas de saída, de maneira a representar uma situação mais próxima do real.

Todos os algoritmos desenvolvidos neste trabalho foram programados em ambiente Matlab, versão 6.5, processados em um microcomputador Pentium 4, 2.40GHz com 512Mb de RAM, sob o sistema operacional Windows XP versão 2002. 


\subsection{PROCEDIMENTO DE IDENTIFICAÇÃO}

O processo de identificação realizado utilizando os modelos ARMAV e ARV, pode ser visto sumarizado por meio dos fluxogramas, ilustrados na Figura 6.1 e 6.2 respectivamente, que mostram os principais passos do algoritmo numérico desenvolvido neste trabalho.

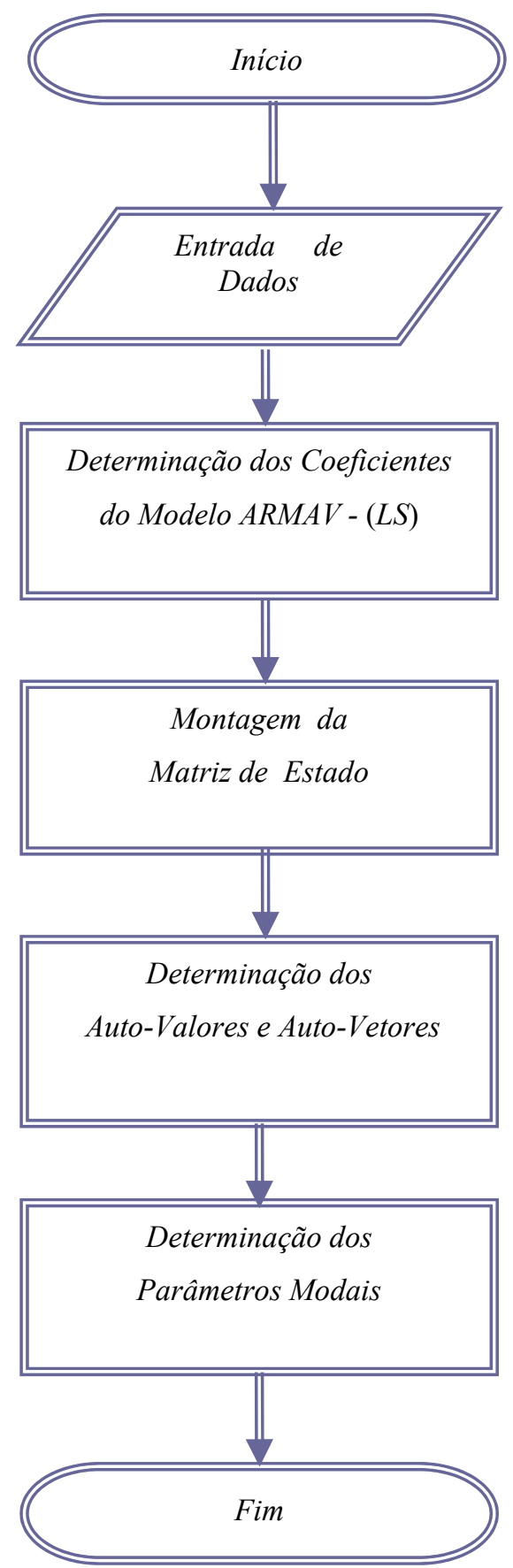

Figura 6.1: Fluxograma do procedimento de identificação - modelo ARMAV 


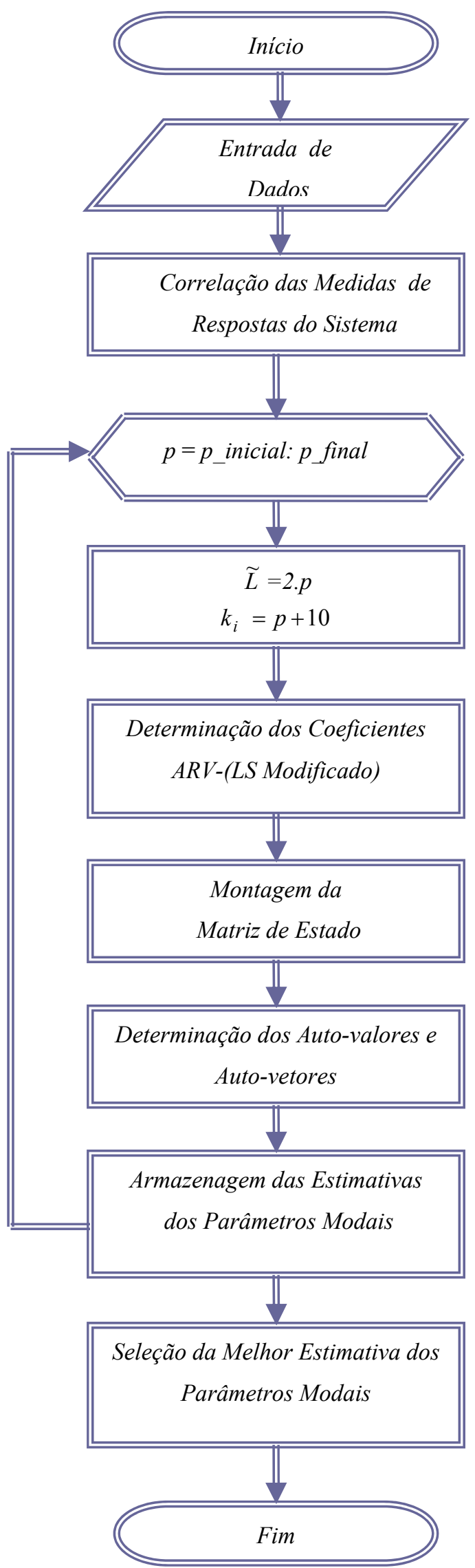

Figura 6.2: Fluxograma do procedimento de identificação - modelo ARV 
Em ambos os modelos, as acelerações foram tomadas como respostas no processo de identificação e geradas a partir de excitações aleatórias múltiplas, não correlacionadas aplicadas em cada uma das massas do sistema. Os dados de respostas simulados foram obtidos a partir do método Runge-Kutta de quarta ordem com condições iniciais de deslocamento e velocidade nulas. A escolha deste tipo de excitação foi adotada em razão de evidenciar melhor a dinâmica do sistema quando utiliza-se o modelo ARMAV, além de corresponder à situação onde a entrada não é conhecida no sentido determinístico mas apenas aproximada por um processo ruído branco.

No fluxograma, correspondente a implementação do modelo ARMAV, (Figura 6.1), a entrada de dados corresponde: aos vetores de forças de excitação em cada massa do sistema, aos vetores de aceleração, ao número de amostras no domínio do tempo $l$, ao número de amostras máxima permitida $L \leq L_{\max } \operatorname{com} L_{\max }=l-p$, o intervalo de amostragem $\Delta t$, a ordem do modelo $p$ estimada pelo critério BIC e a freqüência de corte estipulada de acordo com a banda de interesse.

Os coeficientes do modelo ARMAV $A_{1} \quad \cdots \quad A_{p} \quad B_{0} \quad \cdots \quad B_{p}$ são estimados através da aproximação dos mínimos quadrados (LS) $\hat{\theta}=\hat{Y} \hat{S}^{+}$dada pela equação 5.7.

A partir da matriz de estado $A_{d}$ (equação 4.33) formada pelos coeficientes autoregressivos $A_{1} \cdots A_{p}$, extrai-se os autovalores e auto-vetores e determinam-se os parâmetros modais, que constituem as freqüências naturais, fatores de amortecimento e modos de vibrar do sistema.

Em geral, a presença de ruído nos dados leva à necessidade da sobredeterminação da ordem do modelo para que seja possível identificar os parâmetros modais do sistema. No entanto, esse procedimento identifica além dos modos estruturais os modos computacionais, provenientes dos graus de liberdade adicionais fornecidos ao modelo matemático, que não representam as propriedades físicas do sistema. A eliminação dos modos computacionais é realizada através de alguns critérios de seleção natural tais como: modos provenientes de autovalores não complexos conjugados, fatores de amortecimento negativos e freqüências maiores que a freqüência de corte estipulada. Além disso, os modos computacionais ainda podem ser eliminados com o auxílio do diagrama de estabilidade das freqüências, uma vez que as freqüências 
correspondentes aos modos computacionais variam fortemente de uma estimativa para outra.

No caso da implementação do método de identificação utilizando o modelo ARV, fluxograma correspondente à Figura 6.2, a entrada de dados é composta somente dos vetores de aceleração, uma vez que as excitações não são medidas. Além disso, é necessário o número de amostras no domínio do tempo $l$, o intervalo de amostragem $\Delta t$ e a frequência de corte adotada.

Após ter-se calculado as matrizes de função de correlação das medidas de respostas, é necessário escolher os parâmetros $\widetilde{L}$ e $k_{i}$ para que as matrizes $\widetilde{S}_{\text {modif }}$ e $\widetilde{U}_{\text {modif }}$ (Equação 5.22) possam ser definidas. Respeitando as condições $k_{i}>p-1$ e a sugestão, de acordo com experiências numéricas, de que $k_{i} \in[p+4, p+14]$, é possível vincular os parâmetros $\widetilde{L}$ e $k_{i}$ com o parâmetro $p$ da seguinte forma: $\widetilde{L}=2 p$ e $k_{i}=p+10$, de maneira que o único parâmetro livre no procedimento de identificação passa a ser $p$.

O algoritmo de identificação é aplicado consecutivamente para valores de $p$ tomados num intervalo pré-definido [ $p_{-}$inicial $p_{-}$final]. Para cada valor de $p$ determinam-se os coeficientes auto-regressivos $A_{1} \cdots A_{p}$ utilizando-se a aproximação dos mínimos quadrados (LS-Modificado), e através dos autovalores e autovetores da matriz de estado estima-se os parâmetros modais. Esses parâmetros, referentes a cada identificação, são armazenados num único arquivo de dados, representado pelo bloco Armazenagem das Estimativas dos Parâmetros Modais.

Em seguida é realizado um procedimento para escolher a melhor estimativa dentre os parâmetros modais identificados e consequentemente ser encontrada a ordem do sistema dinâmico. O algoritmo proposto neste trabalho é desenvolvido com base na informação de que os modos estruturais tendem a se estabilizar em determinados intervalos dentro da faixa de freqüência considerada, enquanto que isso já não ocorre com os modos computacionais. As etapas do procedimento computacional podem ser vistas através do fluxograma na (Figura 6.3) que corresponde ao bloco Seleção da Melhor Estimativa dos Parâmetros Modais (Figura 6.2). 


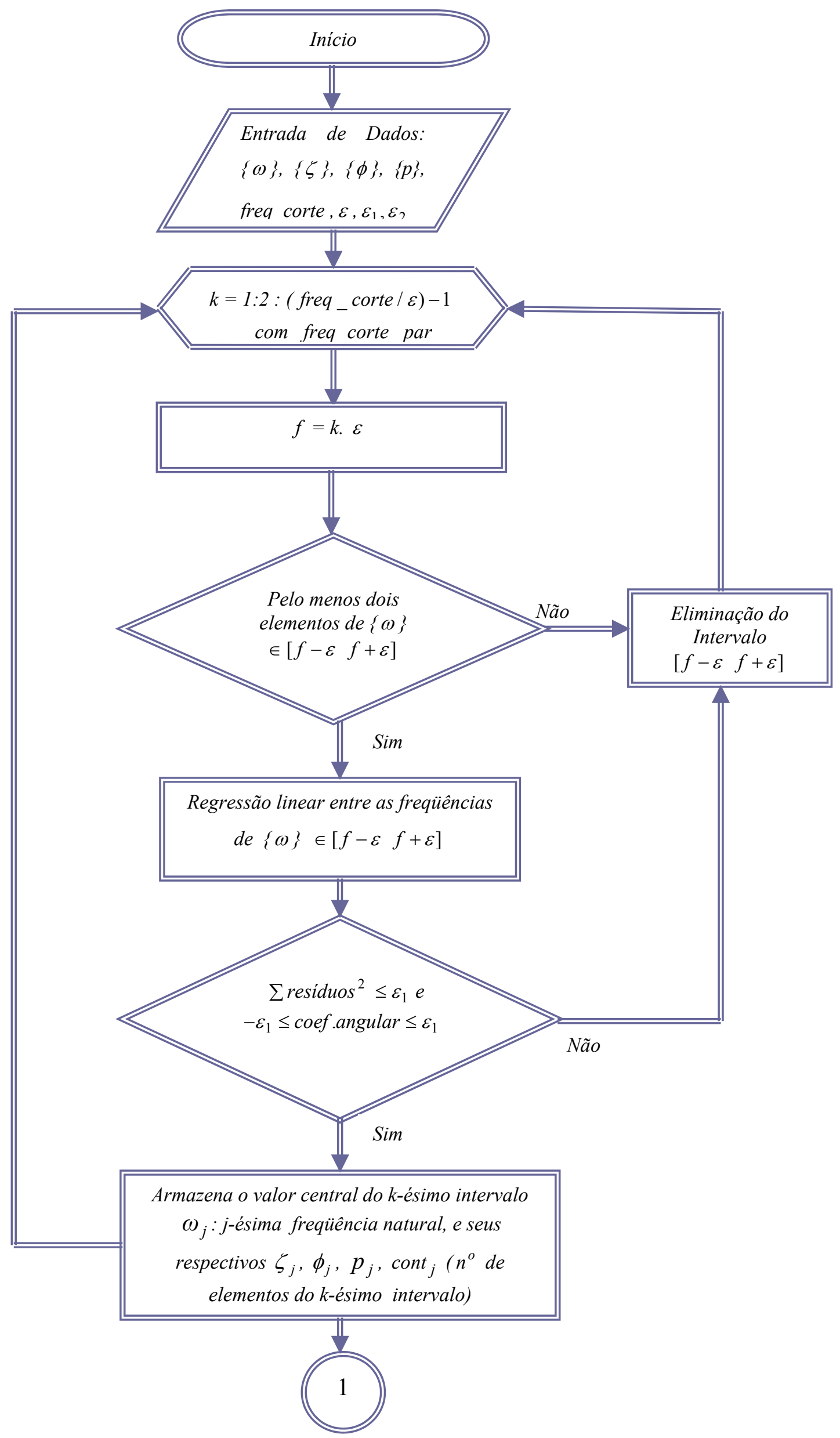




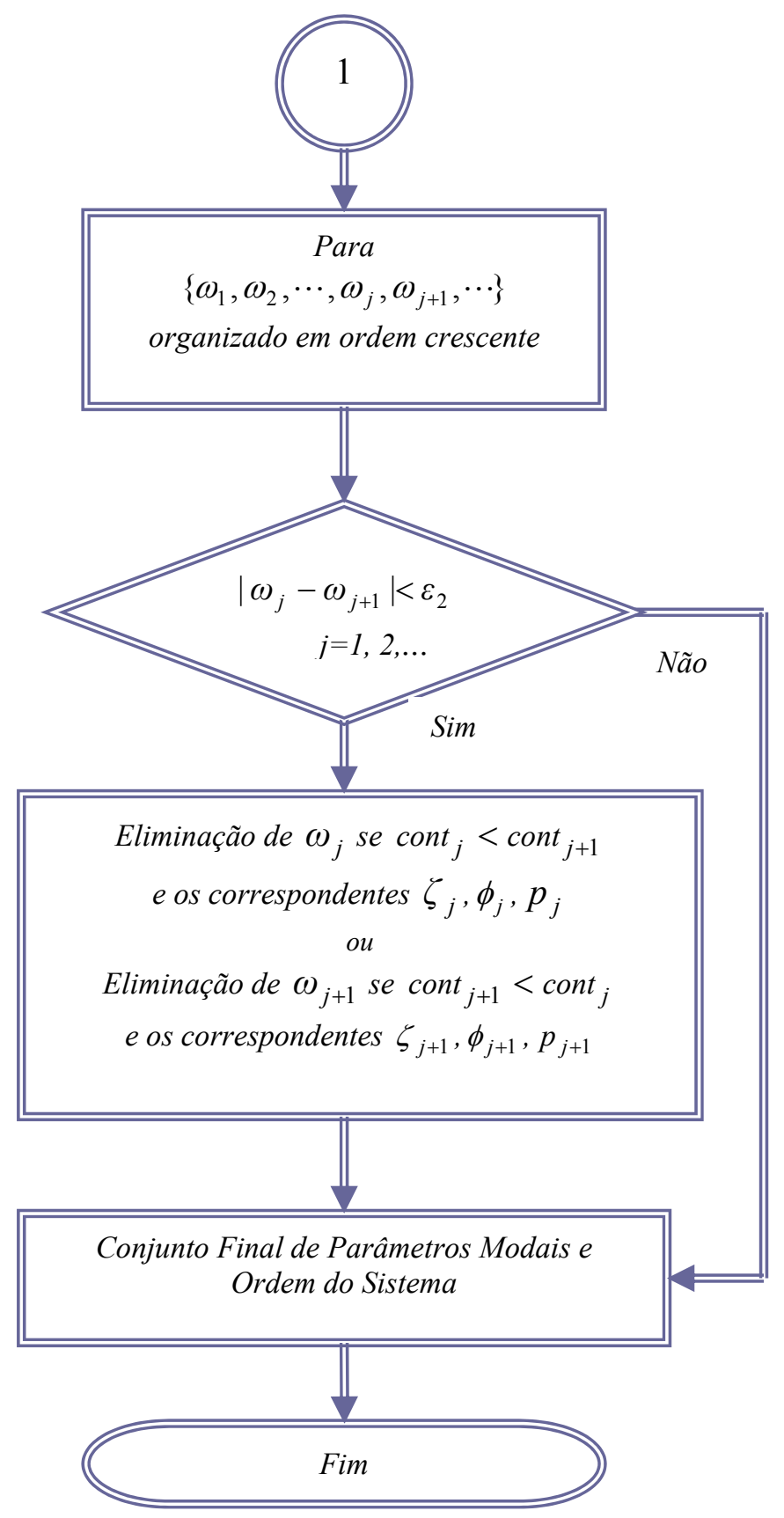

Figura 6.3: Fluxograma da seleção das melhores estimativas

O critério analítico formulado neste trabalho para encontrar uma estimativa dos parâmetros modais do sistema é realizado a partir de um conjunto de freqüências $\{\omega\}$, amortecimentos $\{\zeta\}$, modos de vibrar $\{\phi\}$ e ordens do modelo $\{p\}$, estimados em várias identificações. $\mathrm{O}$ intervalo [ 0 freq_corte $]$ que contém todas as freqüências identificadas em Hertz, onde freq_corte representa a freqüência de corte pré-determinada, é subdividido em intervalos $[f-\varepsilon f+\varepsilon]$ onde $f$ é o centro do intervalo, dado por $f=k . \varepsilon$, e $\varepsilon$ é um valor pré-estabelecido que representa a metade do comprimento do intervalo, com $k=1,3,5, \cdots,($ freq_corte $/ \varepsilon)-1$. O algoritmo que se baseia na 
regressão linear das freqüências pertencentes a cada intervalo de comprimento $2 \varepsilon$, que contém no mínimo 2 elementos, caracteriza-se pela estabilidade ou não das freqüências identificadas. Verifica-se, para cada intervalo, se o somatório dos erros ao quadrado e o coeficiente angular da reta são menores do que $\varepsilon_{1}$ (valor pequeno e pré-definido), condições essas que garantem a estabilidade da freqüência dentro do intervalo analisado. Satisfeitas essas condições, escolhe-se o elemento central de cada intervalo como sendo as freqüências naturais do sistema. Resgatando seus respectivos fatores de amortecimento, modos de vibrar e os valores de $p$, que estão armazenados, determinamse os parâmetros modais. Em seguida, para garantir a não existência de dados redundantes no conjunto final dos parâmetros modais, verifica-se a partir da organização das freqüências em ordem crescente, se a diferença em módulo das freqüências consecutivas, duas a duas, é menor do que um valor pequeno prédeterminado $\varepsilon_{2}$, ou seja, $\mid \omega_{j}-\omega_{j+1} k \varepsilon_{2}$. Se essa condição for satisfeita, elimina-se a freqüência advinda do intervalo que contém o menor número de elementos, seus respectivos fatores de amortecimento, modos de vibrar e valores de $p$.

Por fim, determina-se o conjunto final dos parâmetros modais, $\omega_{j}, \zeta_{j}, \phi_{j}, p_{j}$ com $j=1,2, \ldots, n$, onde $n$ representa o número de graus de liberdade do sistema dinâmico, e consequentemente, a ordem que melhor representa o modelo dinâmico, na faixa operacional de freqüência, que é igual a $2 n$.

\subsection{SIMULAÇÕES NUMÉRICAS DE SISTEMAS DINÂMICOS}

Um sistema dinâmico de $n$ graus de liberdade pode ser representado por um modelo discreto de parâmetros concentrados como mostra a Figura 6.4. Neste modelo considera-se que as molas assumem um comportamento linear, sendo os seus amortecimentos viscosos.

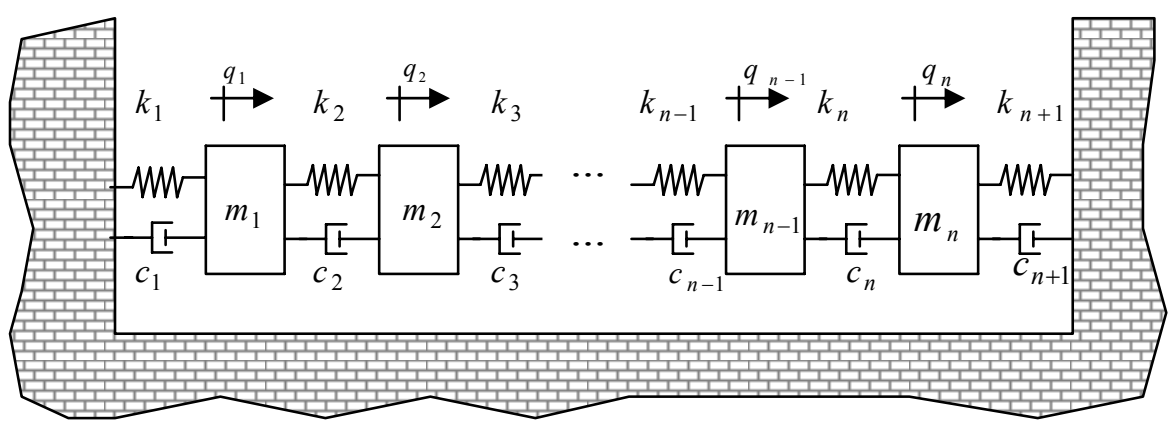

Figura 6.4: Sistema massa - mola - amortecedor de $n$ gdl. 
O movimento horizontal dos $n$ pontos de massa é descrito através do seguinte sistema constituído por $n$ equações diferenciais ordinárias de $2^{\mathrm{a}}$ ordem, como visto na seção 3.2:

$$
M \ddot{w}(t)+C \dot{w}(t)+K w(t)=f(t)
$$

As matrizes de massa, rigidez e amortecimento do modelo acima possuem dimensões $n \times n$ e são dadas por:

$$
\begin{aligned}
& M=\left[\begin{array}{ccccc}
m_{1} & 0 & 0 & 0 & 0 \\
0 & m_{2} & 0 & 0 & 0 \\
0 & 0 & \ddots & 0 & 0 \\
0 & 0 & 0 & m_{n-1} & 0 \\
0 & 0 & 0 & 0 & m_{n}
\end{array}\right] \quad C=\left[\begin{array}{ccccc}
c_{1}+c_{2} & -c_{2} & 0 & 0 & 0 \\
-c_{2} & c_{2}+c_{3} & -c_{3} & 0 & 0 \\
0 & -c_{3} & \ddots & \ddots & 0 \\
0 & 0 & \ddots & c_{n-1}+c_{n} & -c_{n} \\
0 & 0 & 0 & -c_{n} & c_{n}+c_{n+1}
\end{array}\right] \\
& K=\left[\begin{array}{ccccc}
k_{1}+k_{2} & -k_{2} & 0 & 0 & 0 \\
-k_{2} & k_{2}+k_{3} & -k_{3} & 0 & 0 \\
0 & -k_{3} & \ddots & \ddots & 0 \\
0 & 0 & \ddots & k_{n-1}+k_{n} & -k_{n} \\
0 & 0 & 0 & -k_{n} & k_{n}+k_{n+1}
\end{array}\right]
\end{aligned}
$$

sendo que os coeficientes $m_{j}$ para $j=1, \cdots, n, c_{j}$ e $k_{j}$ para $j=1, \cdots, n+1$ são características próprias da estrutura em estudo.

Diversas simulações computacionais foram realizadas para dois sistemas mecânicos diferentes. Ambos apresentam amortecimento não-proporcional e, portanto, todas as freqüências naturais e fatores de amortecimento, apresentados neste trabalho, foram calculados de forma aproximada.

Para simular os possíveis distúrbios presentes nos dados de respostas devido a imprecisões computacionais e interferências nos sensores de medidas, além da análise realizada em dados livres de ruídos, foram adicionados às medidas de respostas $5 \%$ de ruído. O nível de ruído fundamentado na relação sinal-ruído foi estabelecido em termos da potência do ruído em relação à potência do sinal resposta, ou seja, através do valor RMS (ruído branco gaussiano) dividido pelo valor RMS (sinal resposta), onde o RMS (sinal resposta) é definido como sendo o maior valor de RMS dentre todos os sinais de resposta considerados do sistema.

A identificação dos sistemas realizada para os dois diferentes modelos que serão apresentados a seguir, através dos métodos vistos no capítulo cinco, tem como objetivo 
avaliar a eficácia das técnicas estudadas e verificar a precisão dos parâmetros modais obtidos através da utilização dos modelos ARMAV e ARV.

Nos dois casos, o algoritmo de identificação utilizando o modelo ARMAV foi realizado a partir de dados de entrada e saída no domínio do tempo, gerados com $l=$ 1000 pontos, amostrados em intervalos no tempo de $\Delta t=0.001$ segundos. Já o processo de identificação estocástica a partir do modelo ARV exige um número de pontos muito maior. Foram gerados arquivos de dados de saída com $l=200.000$ pontos com intervalo no tempo de $\Delta t=0.0001$ segundos. Em ambos os casos, a identificação foi realizada a partir de uma decimação que reduziu o número de pontos para $l=100.000$ discretizados em intervalos de $\Delta t=0.0002$ segundos, sendo que no segundo caso, além da decimação foi possível realizar a identificação com o número de pontos reduzido para $l$ $=80.000$. O intervalo de amostragem $\Delta t$ foi escolhido como sendo no mínimo dez vezes menor do que o inverso da freqüência máxima do sistema analisado.

\subsubsection{SISTEMA MECÂNICO DE 4 GDL $-1^{\circ} \mathrm{CASO}$}

O primeiro caso consiste na identificação dos parâmetros modais de um sistema mecânico de 4 graus de liberdade com amortecimento viscoso linear não proporcional, caracterizado por freqüências distantes e fatores de amortecimento grandes.

A Tabela 6.1 apresenta os parâmetros físicos, onde $m_{j}$ representa os coeficientes da matriz de massa, $k_{j}$ da matriz de rigidez e $c_{j}$ da matriz de amortecimento, bem como as freqüências naturais $\omega_{j}$ e os fatores de amortecimento $\zeta_{j}$ correspondentes ao $j$-ésimo modo de vibrar do sistema.

Tabela 6.1: Parâmetros físicos e parâmetros modais teóricos - modelo 4gdl

\begin{tabular}{|c||c|c|c|c|}
\hline$m_{j}(\mathrm{~kg})$ & $c_{j}(\mathrm{~N} /(\mathrm{m} / \mathrm{s}))$ & $k_{j}(\mathrm{~N} / \mathrm{m})$ & $\omega_{j}(\mathrm{~Hz})$ & $\zeta_{j}$ \\
\hline \hline 5 & 350 & 105000 & 12.25 & 0.1153 \\
\hline \hline 20 & 450 & 520000 & 30.36 & 0.1373 \\
\hline 10 & 150 & 250000 & 61.44 & 0.2218 \\
\hline 5 & 600 & 710000 & 78.46 & 0.2774 \\
\hline \hline & 650 & 180000 & & \\
\hline
\end{tabular}


Já a Tabela 6.2 mostra os modos de vibrar do sistema simulado, normalizados de maneira que o elemento de maior valor em módulo tenha norma 1.

Tabela 6.2 : modos de vibrar teóricos - modelo $4 \mathrm{gdl}$

\begin{tabular}{|c|c|c|c|}
\hline \multicolumn{4}{|c|}{ Modos Teóricos } \\
\hline \hline Modo 1- $\left(\phi_{1}\right)$ & Modo 2- $\left(\phi_{2}\right)$ & Modo 3- $\left(\phi_{3}\right)$ & Modo 4- $\left(\phi_{4}\right)$ \\
\hline \hline $0.8739-0.0223 \mathrm{i}$ & $-0.5016+0.1071 \mathrm{i}$ & $1.0000+0.0000 \mathrm{i}$ & $-0.0212+0.0148 \mathrm{i}$ \\
$1.0000+0.0000 \mathrm{i}$ & $-0.4343+0.0627 \mathrm{i}$ & $-0.2273+0.0358 \mathrm{i}$ & $0.0250-0.0158 \mathrm{i}$ \\
$0.7928-0.0360 \mathrm{i}$ & $1.0000+0.0000 \mathrm{i}$ & $0.0184+0.0035 \mathrm{i}$ & $-0.4421+0.1113 \mathrm{i}$ \\
$0.6533-0.0553 \mathrm{i}$ & $0.9892-0.1046 \mathrm{i}$ & $0.0647-0.0281 \mathrm{i}$ & $1.0000+0.0000 \mathrm{i}$ \\
\hline
\end{tabular}

Com o intuito de verificar a influência do tamanho da amostra no processo de identificação dos parâmetros modais, os testes simulados através do algoritmo que utiliza o modelo ARMAV foram realizados para valores de $L=500$ e $L=L_{\max }$, sendo $L_{\max }=l-p$, o que corresponde à identificação com amostras de $500+p$ e 1000 pontos, respectivamente.

O resultado das identificações processadas utilizando o modelo ARMAV para os diferentes tamanhos de amostras, com valores de $p$ variando no intervalo [2 20] e com a banda de freqüência estipulada no intervalo [0 freq_corte ], para freq_corte $=85 \mathrm{~Hz}$, pode ser visto na Figura 6.5, através do diagrama de estabilidade em relação às freqüências. Na construção deste diagrama (Figura 6.5) foram consideradas as quatro medidas de respostas, livres e corrompidas por ruído, obtidas a partir de excitações múltiplas em cada uma das massas do sistema.

A análise gráfica, no caso da identificação realizada a partir de dados livres de ruído, revela que as quatro freqüências naturais do sistema se estabilizam em toda faixa de variação de $p$, o que mostra que a ordem mínima do modelo para esse caso é $p=2$. Já para os dados corrompidos por ruído, a ordem mínima passa a ser $p=3$, uma vez que a estimativa da freqüência mais baixa do sistema igual a $12.25 \mathrm{~Hz}$ é identificada somente a partir desse valor. O resultado concorda com o princípio de que o modelo adequado para obter boas estimativas é descrito por um modelo $\operatorname{ARMAV}(p, p)$ com p. $m \geq 2 n$, onde $m$ é o número de saídas e $n$ é o número de graus de liberdade do sistema, que neste caso, corresponde a $m=4$ e $n=4$. 

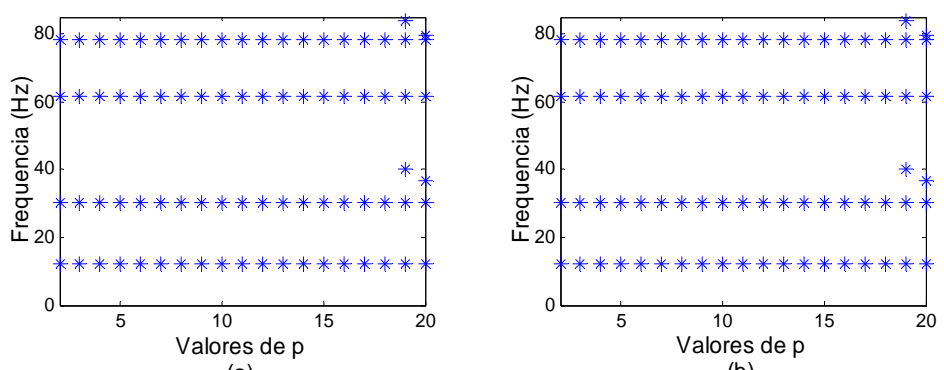

(a)

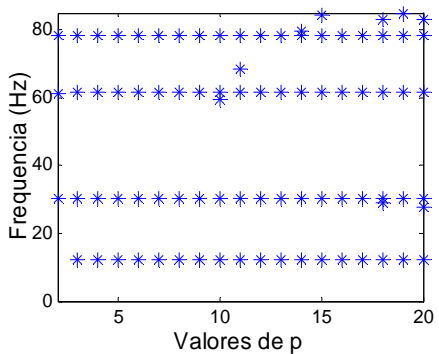

(b)

(c)

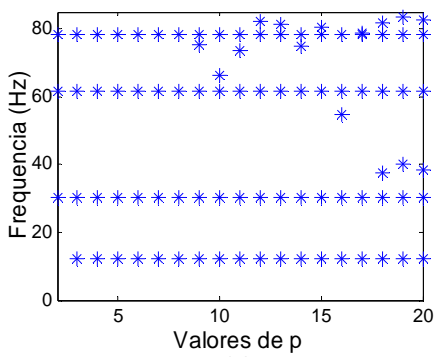

(d)

Figura 6.5: Diagramas de estabilidade - modelo ARMAV - (caso 1): (a) ruído 0\% ( $L=500$ ); (b) ruído $0 \%\left(L=L_{\max }\right)$; (c) ruído 5\% ( $L=500$ ); (d) ruído 5\% ( $\left.L=L_{\max }\right)$

No entanto, a ordem do modelo ARMAV, para todas as simulações numéricas realizadas nesse trabalho, foi estabelecida a partir do critério BIC. A Figura 6.6 exibe um gráfico dos valores de BIC em função dos valores de $p$, obtidos a partir da identificação correspondente ao caso visto na Figura 6.5-(c).

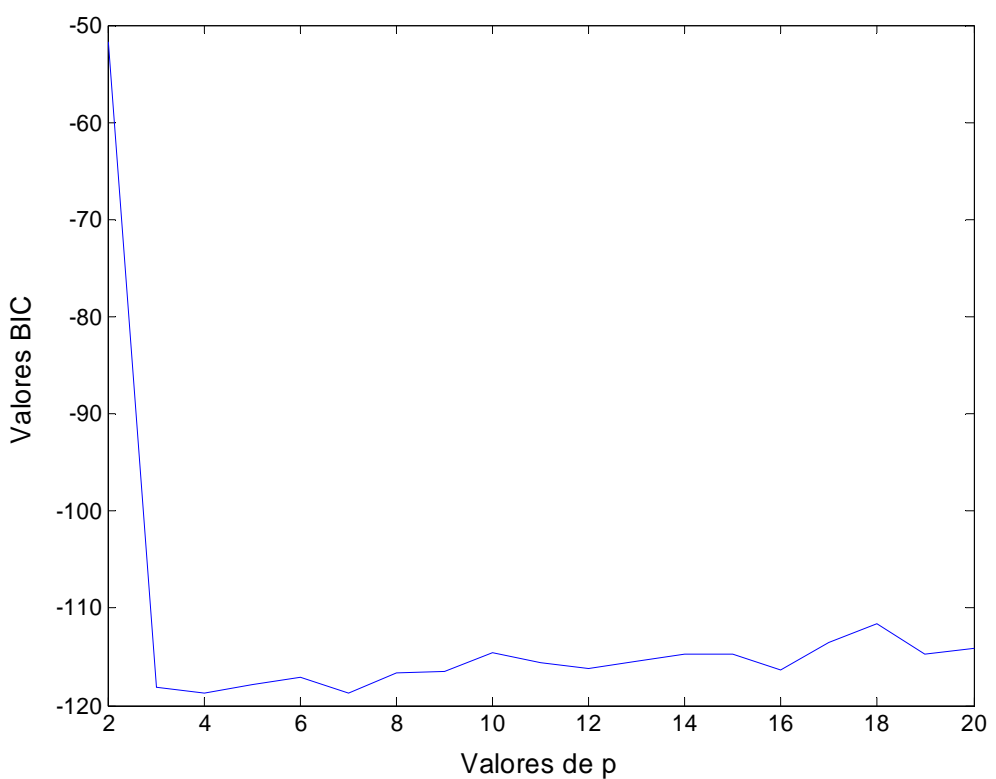

Figura 6.6: Critério BIC aplicado ao modelo ARMAV - (caso 1) 
Segundo esse critério, a ordem ideal corresponde ao ponto de mínimo da curva, que neste caso equivale a $p=4$. Como pode ser observada, a ordem obtida pelo critério BIC é maior comparada com aquela visualizada através do diagrama de estabilidade, proporcionando um modelo ainda mais sobre-determinado.

Os resultados da identificação utilizando as quatro medidas de respostas coletadas simultaneamente contaminadas por 5\% de ruído, tais como: as freqüências naturais, os fatores de amortecimento com seus respectivos erros relativos percentuais em relação aos valores teóricos encontram-se na Tabela 6.3. Essa identificação foi processada a partir do modelo de ordem 4, ou seja $\operatorname{ARMAV}(4,4)$, estabelecido pelo critério BIC, utilizando uma amostra com 1000 pontos, o que corresponde a $L=L_{\max }$.

Tabela 6.3: Parâmetros modais identificados - modelo ARMAV - (caso 1)

\begin{tabular}{|c|c|c|c|}
\hline \multicolumn{4}{|c|}{ Ruido-5\% $\left(L=L_{\max }\right)-\operatorname{ARMAV}(4,4)$} \\
\hline \hline$\omega_{j}(\mathrm{~Hz})$ & Erro $(\%)$ & $\zeta_{j}$ & $\operatorname{Erro}(\%)$ \\
\hline \hline 12.25 & 0.0160 & 0.1305 & 13.21 \\
\hline \hline 30.35 & 0.0457 & 0.1453 & 5.845 \\
\hline \hline 61.42 & 0.0399 & 0.2193 & 1.151 \\
\hline \hline 78.47 & 0.0108 & 0.2785 & 0.3831 \\
\hline
\end{tabular}

Nas identificações onde foram considerados os dados livres de ruído tanto para as amostras com $500+p$ ou 1000 pontos, ou no caso onde foram adicionados ruídos de $5 \%$ as medidas de respostas com $500+p$ pontos, os erros relativos percentuais das freqüências e dos amortecimentos permaneceram iguais aos da Tabela 6.3, não ultrapassando $0.05 \%$ no caso da freqüência e inferior a $14 \%$ para o fator de amortecimento. Isso mostra que, neste caso, onde todas as respostas foram medidas, o tamanho da amostra e a perturbação dos dados por ruído não interferiram nas estimativas das freqüências naturais e fatores de amortecimento do sistema simulado.

As Tabelas 6.4 e 6.5 apresentam os modos de vibrar do sistema, descritos numericamente através de seus auto-vetores, e os erros relativos percentuais correspondentes. Os modos de vibrar descritos na Tabela 6.4 foram identificados utilizando uma amostra de 1000 pontos. Para ambos os casos, onde os dados de saída 
foram considerados livres e corrompidos por ruído, a identificação foi realizada através do modelo ARMAV $(4,4)$.

Tabela 6.4: Modos de vibrar identificados - modelo ARMAV - (caso 1)

\begin{tabular}{|c|c|c|c|}
\hline \multicolumn{4}{|c|}{ Modos Identificados - Ruido 0\% - $\left(L=L_{\max }\right)$} \\
\hline \hline Modo 1- $\left(\phi_{1}\right)$ & Modo 2- $\left(\phi_{2}\right)$ & Modo 3 - $\left(\phi_{3}\right)$ & Modo 4 $-\left(\phi_{4}\right)$ \\
\hline $0.8743-0.0210 \mathrm{i}$ & $-0.5064+0.0889 \mathrm{i}$ & $1.0000+0.0000 \mathrm{i}$ & $-0.0226+0.0127 \mathrm{i}$ \\
$1.0000+0.0000 \mathrm{i}$ & $-0.4367+0.0497 \mathrm{i}$ & $-0.2257+0.0428 \mathrm{i}$ & $0.0267-0.0132 \mathrm{i}$ \\
$0.7939-0.0520 \mathrm{i}$ & $1.0000+0.0000 \mathrm{i}$ & $0.0189-0.0018 \mathrm{i}$ & $-0.4447+0.1067 \mathrm{i}$ \\
$0.6452-0.0679 \mathrm{i}$ & $0.9907-0.1008 \mathrm{i}$ & $0.0612-0.0346 \mathrm{i}$ & $1.0000+0.0000 \mathrm{i}$ \\
\hline \hline \multicolumn{3}{|c}{ Modos Identificados - Ruido 5\% - $\left(L=L_{\max }\right)$} \\
\hline Modo 1- $\left(\phi_{1}\right)$ & Modo2- $\left(\phi_{2}\right)$ & Modo 3- $\left(\phi_{3}\right)$ & Modo 4- $\left(\phi_{4}\right)$ \\
\hline \hline $0.8721-0.0216 \mathrm{i}$ & $-0.4975+0.0838 \mathrm{i}$ & $1.0000+0.0000 \mathrm{i}$ & $-0.0190+0.0110 \mathrm{i}$ \\
$1.0000+0.0000 \mathrm{i}$ & $-0.4281+0.0451 \mathrm{i}$ & $-0.2341+0.0376 \mathrm{i}$ & $0.0301-0.0147 \mathrm{i}$ \\
$0.7904-0.0533 \mathrm{i}$ & $1.0000+0.0000 \mathrm{i}$ & $0.0123-0.0026 \mathrm{i}$ & $-0.4397+0.1040 \mathrm{i}$ \\
$0.6483-0.0697 \mathrm{i}$ & $0.9911-0.1002 \mathrm{i}$ & $0.0550-0.0391 \mathrm{i}$ & $1.0000+0.0000 \mathrm{i}$ \\
\hline \hline
\end{tabular}

Através dos erros na Tabela 6.5 verifica-se que, diferentemente das freqüências e dos fatores de amortecimento, os modos sofreram alterações, ainda que pequenas, de acordo com a presença ou não de ruído nas medidas de respostas e do tamanho da amostra. A técnica mostrou um bom desempenho também na identificação dos modos de vibrar, cujos erros relativos percentuais permaneceram inferiores a $2.5 \%$.

Tabela 6.5: Erros dos modos identificados - modelo ARMAV - (caso 1)

\begin{tabular}{|c||c|c|c||}
\hline \multicolumn{4}{|c|}{ Erro $(\%)-$ Modos Identificados } \\
\hline \hline $\begin{array}{c}\text { Ruido 0\% } \\
(L=500)\end{array}$ & $\begin{array}{c}\text { Ruído 0\% } \\
\left(L=L_{\max }\right)\end{array}$ & $\begin{array}{c}\text { Ruído 5\% } \\
(L=500)\end{array}$ & $\begin{array}{c}\text { Ruido 5\% } \\
\left(L=L_{\max }\right)\end{array}$ \\
\hline \hline 0.1311 & 0.1311 & 0.9357 & 0.2078 \\
\hline 0.1005 & 0.1005 & 2.217 & 0.4276 \\
\hline \hline 0.0261 & 0.0261 & 0.3204 & 0.1249 \\
\hline \hline 0.0654 & 0.0654 & 0.8859 & 0.4305 \\
\hline
\end{tabular}

Para calcular os erros apresentados na Tabela 6.5 e desenhar a forma dos modos mostrados na Figura 6.7, foi necessário fazer a conversão dos modos complexos para a forma real. A conversão foi realizada considerando os módulos de cada componente dos 
auto-vetores descritos na Tabela 6.4. O sinal de cada elemento dos modos reais foi obtido, através da seguinte regra:

$$
\text { sinal }=\left\{\begin{array}{lll}
+ & \text { se } & 3 \pi / 2 \leq \beta<\pi / 2 \\
- & \text { se } & \pi / 2 \leq \beta<3 \pi / 2
\end{array}\right.
$$

onde $\beta$ corresponde ao ângulo de fase de cada componente do auto-vetor.

O erro relativo dos modos foi calculado por: Erro $=\frac{\left|A_{\mathrm{MT}}-A_{\mathrm{MI}}\right|}{A_{\mathrm{MT}}}$, onde $A_{\mathrm{MT}}$ área sob a curva obtida pelos modos reais teóricos e $A_{\mathrm{MI}}$ a área sob a curva alcançada pelos modos reais identificados.

Os dois sistemas massa-mola-amortecedor, que foram utilizados nas simulações, são considerados engastados em ambos os lados, conforme Figura 6.4. Desta forma, em todos os gráficos da representação dos modos deste capítulo, os pontos correspondentes as abcissas 0 e 5, para o caso do sistema mecânico de 4 graus de liberdade, e 0 e 6 para o caso de 5 graus de liberdade, não foram identificados, mas sim adotados como sendo de amplitude zero.
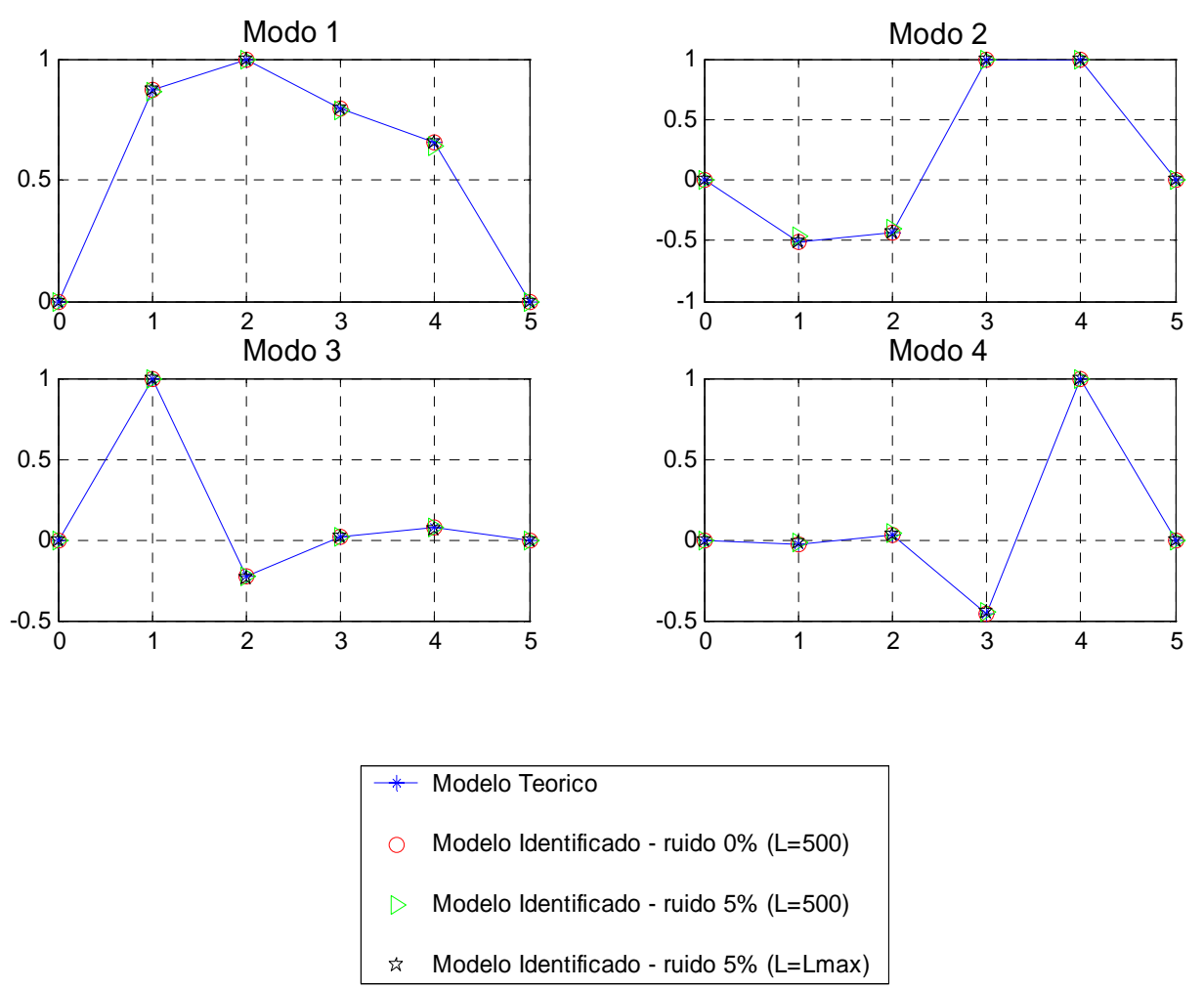

Figura 6.7: Comparação entre as formas dos modos de vibrar teóricos e identificados modelo ARMAV - (caso 1$)$ 
Além do critério do erro relativo, empregado para qualificar os resultados da identificação, utilizou-se também o índice de confiança MAC (Critério de Correlação Modal) para quantificar numericamente a concordância entre os modos de vibrar teóricos $\phi_{T_{j}}$ e identificados $\phi_{I_{j}}$. O índice MAC (ALLEMANG e BROWN, 1983) mede o grau de correlação existente entre as formas dos modos comparados, e é definido por:

$$
\operatorname{MAC}\left(\phi_{I_{j}}, \phi_{T_{j}}\right)=\frac{\left|\phi_{I_{j}}^{*} \phi_{T_{j}}\right|^{2}}{\phi_{I_{j}}^{*} \phi_{I_{j}} \phi_{T_{j}}^{*} \phi_{T_{j}}}
$$

onde $\phi_{j}^{*}$ representa o complexo conjugado de $\phi_{j}$.

Os valores numéricos que o MAC pode assumir variam entre 0 e 1 . Considera-se que existe uma boa correlação entre a forma dos dois modos se o MAC assume um valor acima de 0.9 , e que as formas modais são totalmente não correlacionadas quando o valor de MAC está abaixo de 0.1 .

Tabela 6.6: Valores assumidos por MAC - modelo ARMAV - (caso 1)

\begin{tabular}{|c||c|c|c||}
\hline \multicolumn{4}{|c|}{ Valores - MAC } \\
\hline \hline $\begin{array}{c}\text { Ruido 0\% } \\
(L=500)\end{array}$ & $\begin{array}{c}\text { Ruído 0\% } \\
\left(L=L_{\max }\right)\end{array}$ & $\begin{array}{c}\text { Ruido 5\% } \\
(L=500)\end{array}$ & $\begin{array}{c}\text { Ruido 5\% } \\
\left(L=L_{\max }\right)\end{array}$ \\
\hline 0.9999 & 0.9999 & 0.9998 & 0.9999 \\
\hline \hline 0.9998 & 0.9998 & 0.9986 & 0.9997 \\
\hline 0.9999 & 0.9999 & 0.9997 & 0.9997 \\
\hline \hline 1.0000 & 1.0000 & 0.9997 & 0.9999 \\
\hline
\end{tabular}

Através dos valores apresentados na Tabela 6.6, pode-se observar, de uma outra forma, que os modos foram muito bem identificados, uma vez que os valores assumidos pelo índice de confiança MAC foram superiores a 0.9 .

Geralmente, em situações reais, poucos pontos são medidos e utilizados no processo de identificação dos parâmetros modais, por razões econômicas, devido ao alto custo dos equipamentos de medição. Neste contexto, foram realizadas também simulações onde apenas algumas saídas foram consideradas. Pode-se observar que a qualidade da identificação depende da quantidade de informações e da localização dos pontos de medidas. A Tabela 6.7 mostra alguns dos resultados alcançados na identificação considerando 2 e 3 pontos de saídas, com nível de ruído igual a 5\%. 
Tabela 6.7: Erros dos parâmetros modais identificados considerando número reduzido de pontos de medição - modelo ARMAV- (caso 1)

\begin{tabular}{|c|c|c|c|}
\hline \multicolumn{4}{|c|}{ Erro $(\%)$ - Parâmetros Modais $\left(L=L_{\max }\right)$ - Ruído $5 \%$} \\
\hline \multicolumn{4}{|c|}{3 pontos de medidas de respostas : $m_{1}, m_{2}$ e $m_{3}$} \\
\hline & $\omega_{j}$ & $\zeta_{j}$ & $\operatorname{Modos}-\left(\phi_{j}\right)$ \\
\hline $\operatorname{ARMAV}(11,11)$ & $\begin{array}{l}0.0160 \\
0.0457 \\
0.0399 \\
0.0108 \\
\end{array}$ & $\begin{array}{c}13.21 \\
5.845 \\
1.151 \\
0.3831 \\
\end{array}$ & $\begin{array}{l}0.1798 \\
0.6210 \\
0.3715 \\
0.8243 \\
\end{array}$ \\
\hline \multicolumn{4}{|c|}{2 pontos de medidas de respostas : $m_{1}$ e $m_{2}$} \\
\hline & $\omega_{j}$ & $\zeta_{j}$ & $\operatorname{Modos}-\left(\phi_{j}\right)$ \\
\hline ARMAV $(14,14)$ & $\begin{array}{l}0.0158 \\
0.0457 \\
0.0399 \\
0.0108\end{array}$ & $\begin{array}{c}13.22 \\
5.845 \\
1.151 \\
0.3831\end{array}$ & $\begin{array}{l}0.1740 \\
0.1042 \\
1.466 \\
37.35\end{array}$ \\
\hline
\end{tabular}

Analisando os dados apresentados na Tabela 6.7, pode-se notar que as freqüências e fatores de amortecimento identificados a partir de 2 e 3 pontos de respostas não sofreram alterações, comparados com os resultados da identificação onde todos os pontos foram considerados (Tabela 6.3). No entanto, para alcançar esses resultados, foi preciso aumentar sensivelmente a ordem do modelo ARMAV, de $p=4$ para $p=11$ e $p=14$, nos casos onde foram avaliadas apenas 2 e 3 saídas. Em relação aos modos de vibrar, os resultados também foram satisfatórios com erros inferiores a $1.5 \%$, exceto para o quarto modo, alcançando um erro de quase $37.5 \%$ quando apenas 2 saídas foram avaliadas. A qualidade da identificação também pode ser verificada através dos valores MAC exibidos na Tabela 6.8. O erro grande em relação ao quarto modo, provavelmente deve-se ao fato da escolha não adequada da localização dos pontos de saídas.

Tabela 6.8: Valores assumidos por MAC - modelo ARMAV - (caso 1) considerando número reduzido de pontos de medição

\begin{tabular}{|c|c|}
\hline \multicolumn{2}{|c|}{ Valores MAC - Ruido 5\% $\left(L=L_{\max }\right)$} \\
\hline $\begin{array}{c}\text { 3 pontos de medidas de } \\
\text { respostas: } m_{1}, m_{2} \text { e } m_{3}\end{array}$ & $\begin{array}{c}\text { 2 pontos de medidas de } \\
\text { respostas: } m_{1} \text { e } m_{2}\end{array}$ \\
\hline 0.9999 & 1.0000 \\
\hline 0.9997 & 1.0000 \\
\hline 0.9996 & 0.9984 \\
\hline 0.9995 & 0.3278 \\
\hline
\end{tabular}


Apesar da análise dos resultados obtidos através do modelo ARMAV apresentar consistência dos parâmetros modais, é interessante que seja feita a validação do modelo. A Figura 6.8 apresenta, como exemplo, o resultado do teste do sinal residual correspondente à saída 1. Através da Figura 6.8(a) pode ser observado que a função de auto-correlação do sinal residual permanece dentro do intervalo de confiança, para atrasos superiores a zero, ou exceto no lag zero, garantindo assim que o sinal residual seja próximo do ruído branco. Já a Figura 6.8(b) indica que o histograma do sinal residual se aproxima de uma distribuição Gaussiana. As mesmas conclusões são válidas para as saídas 2, 3 e 4. Assim pode-se dizer que o modelo $\operatorname{ARMAV}(4,4)$, obtido a partir das 4 medidas de respostas com $500+p$ pontos contaminadas por ruído, representa um bom ajuste dos dados pelos quais ele foi estimado.

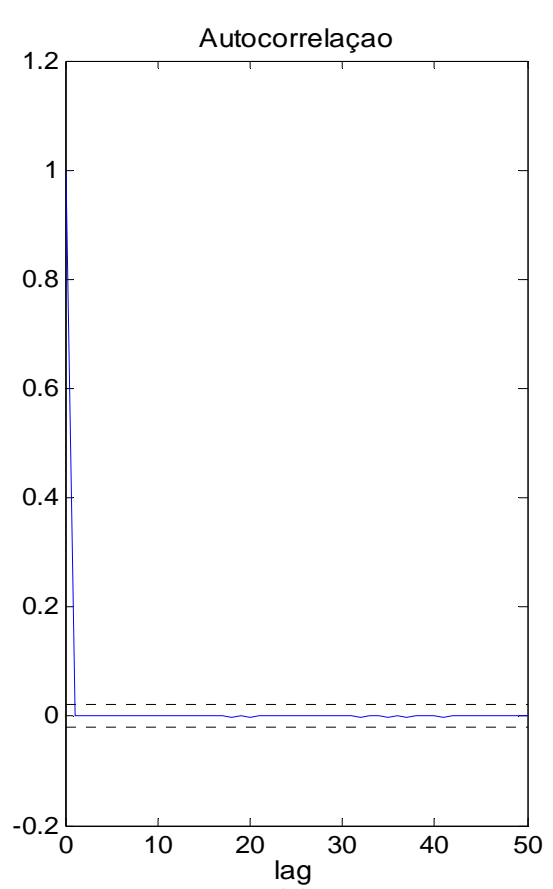

(a)

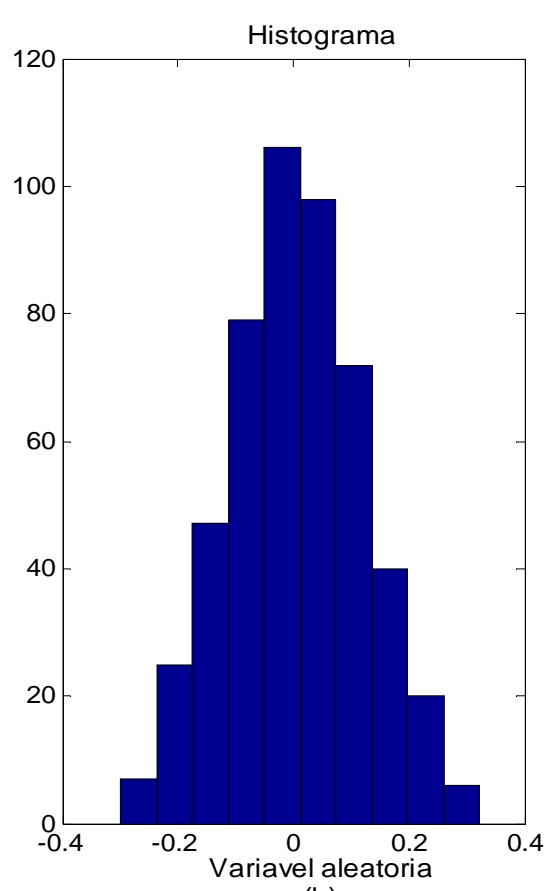

(b)

Figura 6.8: Teste do sinal residual da saída 1

(a) função de auto-correlação; (b) histograma - (caso 1)

No caso da identificação estocástica realizada através do modelo ARV, a ordem do sistema dinâmico foi estimada sem a utilização dos critérios já apresentados. O critério analítico proposto, que contribui para solucionar o problema da determinação da ordem que melhor representa o sistema, dentro de uma faixa de freqüência, é descrito através do algoritmo apresentado na Figura 6.3. 
As estimativas das freqüências obtidas através do modelo ARV, cujo algoritmo está representado no fluxograma da Figura 6.2, pode ser visto na Figura 6.9. Esses resultados foram alcançados por meio da identificação processada a partir das quatro medidas de respostas com 100.000 pontos, livres e contaminados por ruído. Com a suspeita de que o tamanho da amostra fosse excessivamente grande sem necessidade, realizaram-se testes com tamanhos menores, onde foi constatado que as freqüências continuavam a ser identificadas de maneira satisfatória, enquanto que os erros em relação aos fatores de amortecimento aumentavam drasticamente.

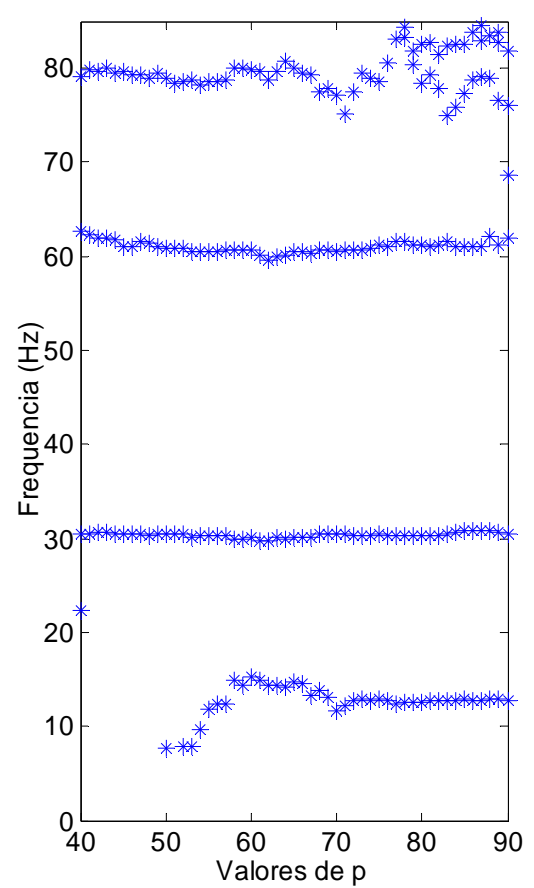

(a)

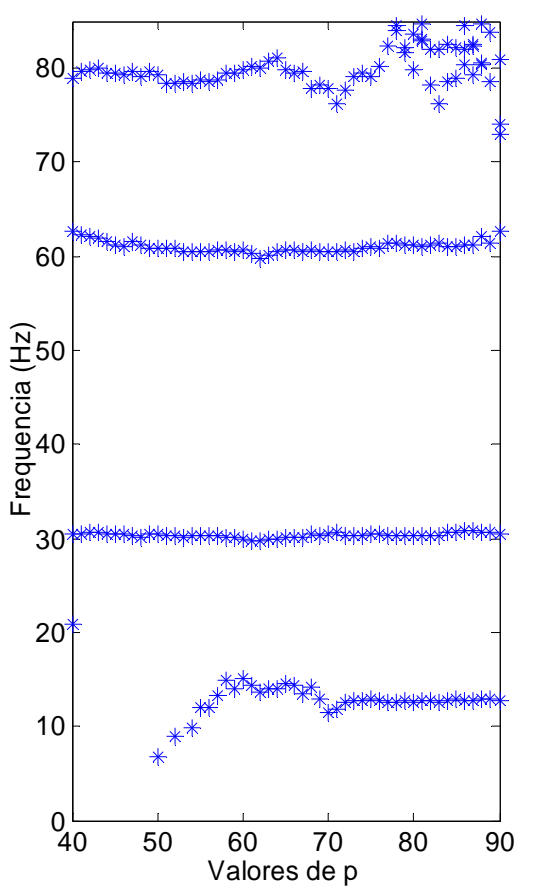

(b)

Figura 6.9: Diagramas de estabilidade - modelo ARV-(caso 1) (a) ruído 0\%; (b) ruído $5 \%$

Através dos gráficos ilustrados na Figura 6.9 pode-se observar a estabilização de quatro freqüências pertencentes à banda de [0 85] Hz no intervalo [40 90] de variação de $p$. Esta faixa de variação foi escolhida após a visualização gráfica do diagrama de estabilidade em uma faixa maior com $p$ variando de 2 a 100.

Nos casos acima, após o conjunto de parâmetros obtidos no processo de identificação, o algoritmo descrito na Figura 6.3 é utilizado para selecionar a melhor estimativa, considerando os seguintes dados: $\varepsilon=0.25, \varepsilon_{1}=0.1 \mathrm{e} \varepsilon_{2}=1$. Os resultados da melhor estimativa dentre as freqüências identificadas com seus respectivos fatores de 
amortecimento e modos de vibrar são mostrados nas Tabelas 6.9 e 6.10 , onde $p_{j}$ representa a ordem do modelo ARV utilizado para identificar a freqüência $\omega_{j}$ e seu correspondente fator de amortecimento $\zeta_{j}$ e modo de vibrar $\phi_{j}$.

Tabela 6.9: Parâmetros modais identificados - modelo ARV- (caso 1)

\begin{tabular}{|c|c|c|c|c||}
\hline \multicolumn{5}{|c|}{ Ruido-0\%-ARV $\left(p_{j}\right)$} \\
\hline \hline$p_{j}$ & $\omega_{j}(\mathrm{~Hz})$ & Erro $(\%)$ & $\zeta_{j}$ & Erro $(\%)$ \\
\hline 82 & 12.73 & 3.924 & 0.1358 & 17.81 \\
\hline \hline 65 & 30.12 & 0.7967 & 0.1484 & 8.070 \\
\hline \hline 65 & 60.52 & 1.508 & 0.2273 & 2.480 \\
\hline \hline 56 & 78.54 & 0.0985 & 0.2489 & 10.29 \\
\hline \hline \multicolumn{5}{|c|}{ Ruido-5\%-ARV $\left(p_{j}\right)$} \\
\hline \hline$p_{j}$ & $\omega_{j}(\mathrm{~Hz})$ & Erro $(\%)$ & $\zeta_{j}$ & Erro $(\%)$ \\
\hline \hline 81 & 12.66 & 3.368 & 0.1281 & 11.15 \\
\hline \hline 65 & 30.11 & 0.8202 & 0.1481 & 7.833 \\
\hline \hline 80 & 61.22 & 0.3704 & 0.2218 & 0.0094 \\
\hline \hline 58 & 79.56 & 1.402 & 0.2449 & 11.70 \\
\hline \hline
\end{tabular}

Tabela 6.10: Modos de vibrar identificados - modelo ARV - (caso 1)

\begin{tabular}{||c|c|c|c||}
\hline \multicolumn{4}{|c||}{ Modos Identificados - Ruido 0\%- ARV $\left(p_{j}\right)$} \\
\hline Modo 1- $\left(\phi_{1}\right)$ & Modo 2- $\left(\phi_{2}\right)$ & Modo 3- $\left(\phi_{3}\right)$ & Modo 4 - $\left(\phi_{4}\right)$ \\
\hline \hline $0.8738-0.0188 \mathrm{i}$ & $-0.4982+0.0989 \mathrm{i}$ & $1.0000+0.0000 \mathrm{i}$ & $0.0506+0.2094 \mathrm{i}$ \\
$1.0000+0.0000 \mathrm{i}$ & $-0.3777+0.0951 \mathrm{i}$ & $-0.1842+0.1557 \mathrm{i}$ & $-0.0151-0.0528 \mathrm{i}$ \\
$0.8026-0.0012 \mathrm{i}$ & $1.0000+0.0000 \mathrm{i}$ & $-0.0217-0.0532 \mathrm{i}$ & $-0.4170+0.2709 \mathrm{i}$ \\
$0.6689-0.0069 \mathrm{i}$ & $0.9853+0.0210 \mathrm{i}$ & $0.0609-0.0122 \mathrm{i}$ & $1.0000+0.0000 \mathrm{i}$ \\
\hline \hline \multicolumn{3}{|c}{ Modos Identificados - Ruido 5\% - ARV $\left(p_{j}\right)$} \\
\hline \hline Modo 1- $\left(\phi_{1}\right)$ & Modo2- $\left(\phi_{2}\right)$ & Modo 3- $\left(\phi_{3}\right)$ & Modo 4- $\left(\phi_{4}\right)$ \\
\hline \hline $0.8721-0.0216 \mathrm{i}$ & $-0.4975+0.0838 \mathrm{i}$ & $1.0000+0.0000 \mathrm{i}$ & $-0.0190+0.0110 \mathrm{i}$ \\
$1.0000+0.0000 \mathrm{i}$ & $-0.4281+0.0451 \mathrm{i}$ & $-0.2341+0.0376 \mathrm{i}$ & $0.0301-0.0147 \mathrm{i}$ \\
$0.7904-0.0533 \mathrm{i}$ & $1.0000+0.0000 \mathrm{i}$ & $0.0123-0.0026 \mathrm{i}$ & $-0.4397+0.1040 \mathrm{i}$ \\
$0.6483-0.0697 \mathrm{i}$ & $0.9911-0.1002 \mathrm{i}$ & $0.0550-0.0391 \mathrm{i}$ & $1.0000+0.0000 \mathrm{i}$ \\
\hline
\end{tabular}

Através da Tabela 6.9 observa-se que as estimativas das freqüências naturais possuem erros relativos percentuais menores do que $4 \%$, enquanto que os valores 
estimados para os fatores de amortecimento apresentam erros inferiores a 18\%. Já em relação aos modos de vibrar, pode ser visto através da Tabela 6.11 que os mesmos foram identificados satisfatoriamente, com erros inferiores a $3.5 \%$, exceto o erro em relação ao quarto modo que atingiu aproximadamente $20.5 \%$.

Tabela 6.11: Erros dos modos identificados -modelo ARV - (caso 1)

\begin{tabular}{|c|c|}
\hline \multicolumn{2}{|c|}{ Erro (\%) - Modos Identificados } \\
\hline \hline Ruido 0\% & Ruido 5\% \\
\hline \hline 0.6644 & 0.3251 \\
\hline \hline 1.474 & 2.232 \\
\hline 1.945 & 3.121 \\
\hline 20.32 & 18.79 \\
\hline
\end{tabular}

Nota-se através dos resultados obtidos que a técnica utilizada na identificação trata de forma eficiente o ruído adicionado aos dados de respostas, uma vez que os erros relativos da freqüência e fatores de amortecimento e modos de vibrar não sofrem mudanças significativas na presença de ruído.

O bom desempenho da técnica empregada na identificação dos parâmetros modais pode ser verificado também através dos índices MAC exibidos na Tabela 6.12 .

Tabela 6.12: Valores assumidos por MAC - modelo ARV -(caso 1)

\begin{tabular}{|c|c|}
\hline \multicolumn{2}{|c|}{ Valores - MAC } \\
\hline \hline Ruido 0\% & Ruido 5\% \\
\hline 0.9992 & 0.9992 \\
\hline 0.9939 & 0.9929 \\
\hline 0.9808 & 0.9810 \\
\hline \hline 0.9485 & 0.9552 \\
\hline
\end{tabular}

A forma dos modos obtidos através do modelo ARV, a partir de dados livres e corrompidos por ruído pode ser vista através dos seguintes gráficos na Figura 6.10. 

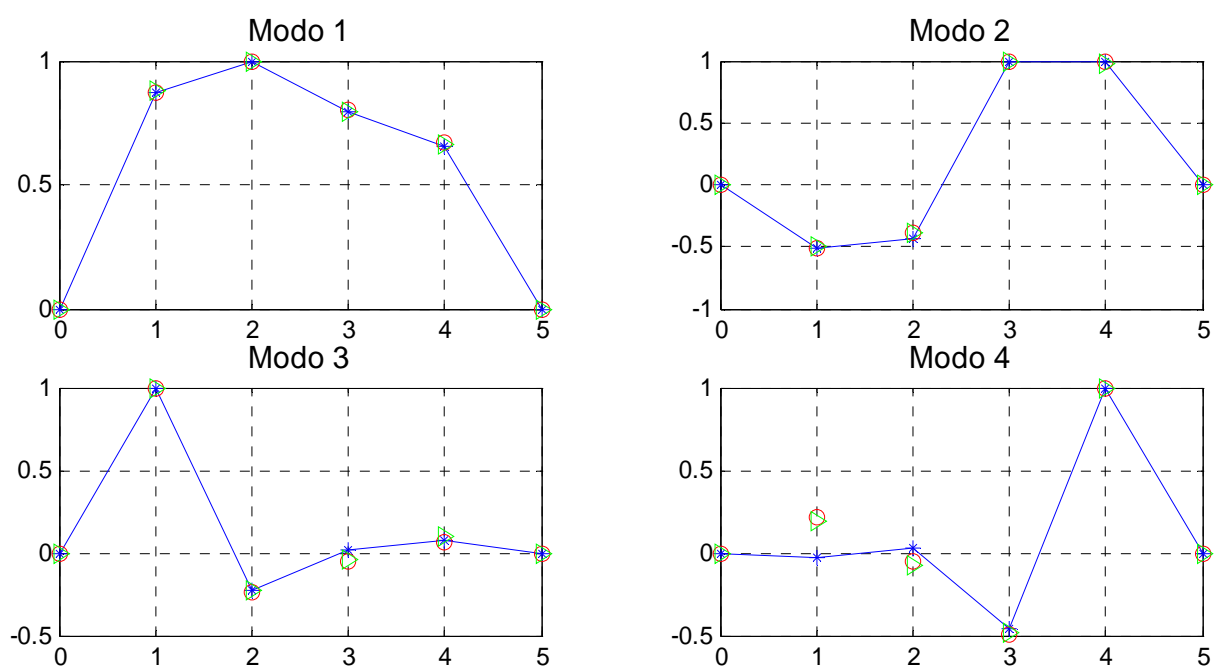

Figura 6.10: Comparação entre as formas dos modos de vibrar teóricos e identificados - modelo ARV - (caso 1)

É importante dizer que para cada modelo diferente, a faixa de variação de $p$ no processo de identificação utilizando essa técnica através do modelo ARV, pode sofrer alterações dependendo da quantidade de respostas medidas e também da adição ou não de ruídos a esses dados.

Tabela 6.13: Erros dos parâmetros modais identificados considerando número reduzido de pontos de medição - modelo ARV- (caso 1$)$

\begin{tabular}{|c||c|c|c|}
\hline \multicolumn{4}{|c|}{ Erro(\%) - Parâmetros Modais-Ruído 5\% } \\
\hline \multicolumn{3}{|c|}{ 3 pontos de medidas de respostas : $m_{1}, m_{2}$ e $m_{3}$} \\
\hline$p_{j}$ & $\omega_{j}$ & $\zeta_{j}$ & $\phi_{j}$ \\
\hline 82 & 2.254 & 5.203 & 0.4594 \\
69 & 2.509 & 1.789 & 0.9555 \\
61 & 0.2422 & 14.63 & 0.9546 \\
- & - & - & - \\
\hline \multicolumn{3}{|c|}{2 pontos de medidas de respostas $: m_{1}$ e $m_{2}$} \\
\hline 176 & 1.635 & 29.24 & 0.7482 \\
178 & 0.0450 & 1.981 & 1.193 \\
158 & 0.2534 & 8.668 & 1.292 \\
- & - & - & - \\
\hline
\end{tabular}


Tabela 6.14: Valores assumidos por MAC - modelo ARV - (caso1) considerando número reduzido de pontos de medição

\begin{tabular}{|c|c|}
\hline \multicolumn{2}{|c|}{ Valores MAC - Ruido 5\% } \\
\hline $\begin{array}{c}\text { 3 pontos de medidas de } \\
\text { respostas: } m_{1}, m_{2} \text { e } m_{3}\end{array}$ & $\begin{array}{c}\text { 2 pontos de medidas de } \\
\text { respostas: } m_{1} \text { e } m_{2}\end{array}$ \\
\hline 0.9985 & 0.9999 \\
\hline 0.9958 & 0.9997 \\
\hline 0.9799 & 0.9929 \\
\hline- & - \\
\hline \hline
\end{tabular}

Para o caso onde somente 3 medidas de respostas são consideradas o intervalo de variação de $p$, necessário para o processo de identificação, corresponde a [40 100]. Ainda assim, como mostra a Tabela 6.13, apesar dos erros em relação às freqüências não ultrapassarem 2.5\%, aos fatores de amortecimento serem inferiores a $15 \%$ e em relação aos modos menores do que $1 \%$, não foi possível identificar a freqüência mais alta do sistema.

Já para a identificação realizada com apenas 2 pontos de saída considerando um intervalo [100 200] para valores de $p$, além de não ser possível encontrar a estimativa para a freqüência mais alta o erro em relação ao amortecimento teve um aumento significativo chegando a quase $29.5 \%$.

Através dos valores MAC, exibidos na Tabela 6.14 pode-se confirmar a identificação satisfatória dos três primeiros modos.

\subsubsection{SISTEMA MECÂNICO DE 5 GDL $-2^{\circ}$ CASO}

Neste caso, as técnicas de identificação através do modelo ARMAV e ARV, estudadas neste trabalho, são aplicadas na análise de um sistema dinâmico estrutural de 5 graus de liberdade, com amortecimento não proporcional. Os parâmetros físicos do sistema simulado, bem como suas freqüências naturais teóricas e seus respectivos fatores de amortecimento encontram-se na Tabela 6.15. Já os modos de vibrar estão representados numericamente na Tabela 6.16 através dos auto-vetores do sistema em sua forma normalizada. 
Tabela 6.15: Parâmetros físicos e parâmetros modais teóricos - modelo 5gdl

\begin{tabular}{|c|c||c||c||c||}
\hline$m_{i}(\mathrm{~kg})$ & $c_{i}(\mathrm{~N} /(\mathrm{m} / \mathrm{s}))$ & $k_{i}(\mathrm{~N} / \mathrm{m})$ & $\omega_{j}(\mathrm{~Hz})$ & $\zeta_{j}$ \\
\hline \hline 0.5 & 1 & 1100 & 3.157 & 0.0068 \\
\hline \hline 2 & 0.3 & 4330 & 9.130 & 0.0083 \\
\hline \hline 1 & 0.5 & 2500 & 18.02 & 0.0110 \\
\hline \hline 0.3 & 0.5 & 3500 & 19.54 & 0.0178 \\
\hline \hline 0.1 & 0.3 & 1300 & 27.41 & 0.0336 \\
\hline & 1 & 1200 & & \\
\hline
\end{tabular}

A finalidade aqui é avaliar a eficiência dessas técnicas de identificação, no caso onde o sistema estrutural é caracterizado por apresentar freqüências naturais próximas e baixos fatores de amortecimento, como mostra a Tabela 6.15 .

Tabela 6.16: Modos de vibrar teóricos - modelo 5gdl

\begin{tabular}{||c|c|c|c|c||}
\hline \multicolumn{5}{|c|}{ Modos Teóricos } \\
\hline Modo 1- $\left(\phi_{1}\right)$ & Modo 2- $\left(\phi_{2}\right)$ & Modo 3- $\left(\phi_{3}\right)$ & Modo 4 $-\left(\phi_{4}\right)$ & Modo 5 - $\left(\phi_{5}\right)$ \\
\hline \hline $0.8274-0.0025 \mathrm{i}$ & $-0.5270+0.0015 \mathrm{i}$ & $1.0000+0.0000 \mathrm{i}$ & $-0.1097+0.0068 \mathrm{i}$ & $0.0012-0.0003 \mathrm{i}$ \\
$1.0000+0.0000 \mathrm{i}$ & $-0.4606-0.0026 \mathrm{i}$ & $-0.2267+0.0032 \mathrm{i}$ & $0.0535-0.0010 \mathrm{i}$ & $-0.6026+0.0005 \mathrm{i}$ \\
$0.9841+0.0006 \mathrm{i}$ & $0.8670+0.0025 \mathrm{i}$ & $-0.0256+0.0061 \mathrm{i}$ & $-0.3098-0.0141 \mathrm{i}$ & $0.0530-0.0078 \mathrm{i}$ \\
$0.8622-0.0003 \mathrm{i}$ & $1.0000+0.0000 \mathrm{i}$ & $0.2119-0.0127 \mathrm{i}$ & $0.7640+0.0598 \mathrm{i}$ & $-0.3594+0.0333 \mathrm{i}$ \\
$0.4555-0.0028 \mathrm{i}$ & $0.5986-0.0111 \mathrm{i}$ & $0.2239-0.0296 \mathrm{i}$ & $1.0000+0.0001 \mathrm{i}$ & $1.0000+0.0001 \mathrm{i}$ \\
\hline
\end{tabular}

Para este caso também serão apresentados alguns resultados da identificação, obtidos através do algoritmo que utiliza o modelo ARMAV, para amostras de tamanhos diferentes com $500+p$ e 1000 pontos.

A Figura 6.11 mostra os gráficos das estimativas das freqüências pertencentes à banda de [0 30] Hz contra o intervalo de variação de $p$ [2 20], onde $30 \mathrm{~Hz}$ corresponde à freqüência de corte pré-estabelecida. As estimativas foram encontradas a partir das cinco medidas de entrada e saída do sistema para diferentes tamanhos de amostras. A partir da análise gráfica dos diagramas torna-se evidente que o sistema analisado possui cinco freqüências naturais visto que estas se estabilizam em toda faixa de variação de $p$, sendo os dados de resposta, livre ou não de ruído, e independentemente do número de pontos utilizado no processo de identificação. Esses resultados mostram a eficiência do método dos mínimos quadrados aliado ao modelo ARMAV em identificar sistemas com freqüências próximas. 


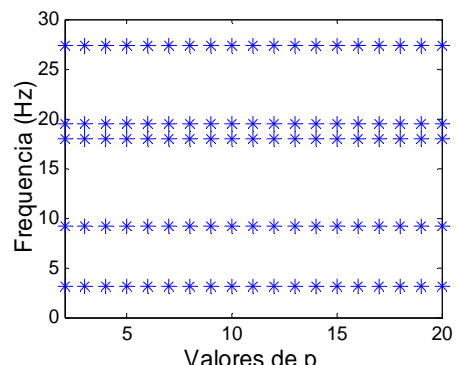

(a)

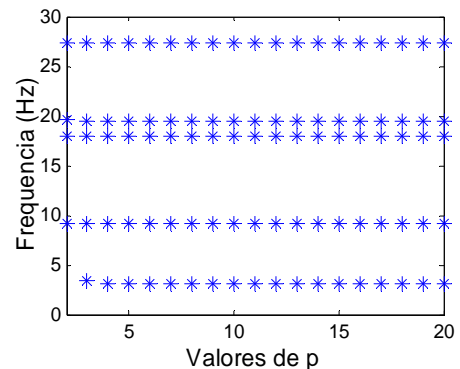

(c)

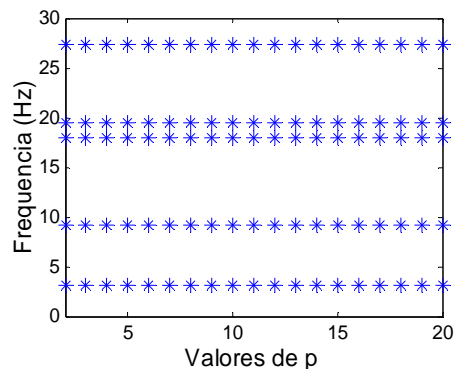

(b)

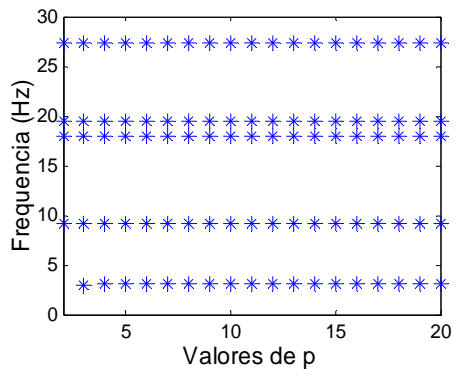

(d)

Figura 6.11: Diagramas de estabilidade - modelo ARMAV- (caso 2): (a) ruído 0\% ( $L=500$ ); (b) ruído $0 \%\left(L=L_{\max }\right)$; (c) ruído 5\% ( $L=500$ ); (d) ruído 5\% ( $\left.L=L_{\max }\right)$

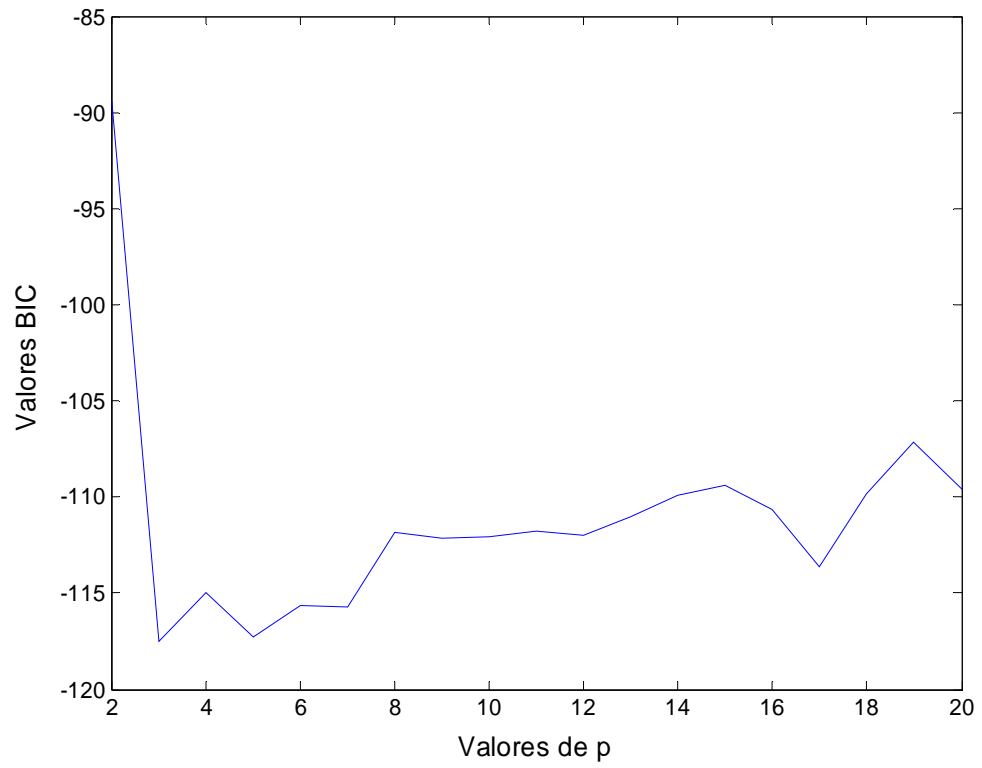

Figura 6.12: Critério BIC aplicado ao modelo ARMAV- (caso2)

Como já dito anteriormente, a ordem de todos os modelos ARMAV, analisados neste trabalho, foi determinada através do uso do critério BIC. No entanto, para este caso, será ilustrado apenas o gráfico da evolução dos valores BIC num determinado intervalo de $p$, para estabelecer a ordem do modelo construído a partir de dados de 
respostas contaminados por ruído com 1000 pontos. Como pode ser visto através da Figura 6.12, o ponto de mínimo da curva que corresponde a ordem do modelo, segundo esse critério, é $p=3$. Entretanto, é possível visualizar através do diagrama (Figura 6.11) que a ordem mínima para realizar uma melhor identificação seria $p=4$, porque é a partir desse valor de $p$ que a estimativa da freqüência natural mais baixa do sistema, igual a $3.15 \mathrm{~Hz}$, se estabiliza. Neste caso, portanto, pode-se verificar que a ordem do modelo determinada através do critério BIC foi sub-estimada.

As estimativas das freqüências naturais e dos fatores de amortecimento obtidas a partir da identificação utilizando as cinco saídas do sistema, com seus correspondentes erros relativos percentuais, são exibidos na Tabela 6.17 .

Tabela 6.17: Parâmetros modais identificados - modelo ARMAV - (caso 2)

\begin{tabular}{|c|c|c|c|c|c|c|c|}
\hline \multicolumn{4}{|c|}{ Ruido-0\% $(L=500)-\mathrm{ARMAV}(5,5)$} & \multicolumn{4}{|c|}{ Ruido-0\% $\left(L=L_{\max }\right)-\operatorname{ARMAV}(3,3)$} \\
\hline$\omega_{j}(\mathrm{~Hz})$ & $\operatorname{Erro}(\%)$ & $\zeta_{j}$ & $\operatorname{Erro}(\%)$ & $\omega_{j}(\mathrm{~Hz})$ & $\operatorname{Erro}(\%)$ & $\zeta_{j}$ & $\operatorname{Erro}(\%)$ \\
\hline 3.157 & 0.0030 & 0.0068 & 0.1070 & 3.157 & 0.0030 & 0.0068 & 0.1070 \\
\hline 9.130 & 0.0008 & 0.0083 & 0.0853 & 9.130 & 0.0008 & 0.0083 & 0.0853 \\
\hline 18.02 & 0.0001 & 0.0110 & 0.0329 & 18.02 & 0.0001 & 0.0110 & 0.0329 \\
\hline 19.54 & 0.0005 & 0.0179 & 0.4194 & 19.54 & 0.0005 & 0.0179 & 0.4194 \\
\hline 27.41 & 0.0003 & 0.0336 & 0.0906 & 27.41 & 0.0003 & 0.0336 & 0.0906 \\
\hline \multicolumn{4}{|c|}{ Ruido-5\% $(L=500)-$ ARMAV $(3,3)$} & \multicolumn{4}{|c|}{ Ruido-5\% $\left(L=L_{\max }\right)-\operatorname{ARMAV}(3,3)$} \\
\hline$\omega_{j}(\mathrm{~Hz})$ & $\operatorname{Erro}(\%)$ & $\zeta_{j}$ & $\operatorname{Erro}(\%)$ & $\omega_{j}(\mathrm{~Hz})$ & $\operatorname{Erro}(\%)$ & $\zeta_{j}$ & $\operatorname{Erro}(\%)$ \\
\hline 3.337 & 5.709 & 0.0063 & 6.692 & 3.001 & 4.916 & 0.0073 & 7.522 \\
\hline 9.129 & 0.0072 & 0.0083 & 0.2908 & 9.130 & 0.0046 & 0.0083 & 0.0663 \\
\hline 18.02 & 0.0063 & 0.0110 & 0.2962 & 18.02 & 0.0052 & 0.0110 & 0.1510 \\
\hline 19.54 & 0.0006 & 0.0179 & 0.5211 & 19.54 & 0.0016 & 0.0179 & 0.5004 \\
\hline 27.41 & 0.0006 & 0.0336 & 0.0929 & 27.41 & 0.0003 & 0.0336 & 0.0905 \\
\hline
\end{tabular}

Como pode ser observado, notou-se alteração nas estimativas para diferentes tamanhos de amostras, somente quando os dados de saída foram acrescidos de ruído. Nestes casos os erros referentes aos valores estimados das freqüências naturais não ultrapassaram $6 \%$ enquanto que para os fatores de amortecimento foram inferiores a $8 \%$.

Os modos de vibrar representados pelos auto-vetores do sistema, identificados através do modelo $\operatorname{ARMAV}(3,3)$ aplicado a uma amostra de 1000 pontos corrompida ou não por ruído são exibidos na Tabela 6.18 . 
Tabela 6.18: Modos de vibrar identificados - modelo ARMAV - (caso 2)

\begin{tabular}{||c|c|c|c||c||}
\hline \multicolumn{5}{|c|}{ Modos Identificados - Ruido 0\% - $\left(L=L_{\max }\right)$} \\
\hline Modo 1 - $\left(\phi_{1}\right)$ & Modo 2 - $\left(\phi_{2}\right)$ & Modo 3 - $\left(\phi_{3}\right)$ & Modo 4- $\left(\phi_{4}\right)$ & Modo 5 - $\left(\phi_{5}\right)$ \\
\hline $0.8274-0.0025 \mathrm{i}$ & $-0.5270+0.0015 \mathrm{i}$ & $1.0000+0.0000 \mathrm{i}$ & $-0.1097+0.0071 \mathrm{i}$ & $0.0012-0.0003 \mathrm{i}$ \\
$1.0000+0.0000 \mathrm{i}$ & $-0.4606-0.0026 \mathrm{i}$ & $-0.2667+0.0032 \mathrm{i}$ & $0.0535-0.0011 \mathrm{i}$ & $-0.0026+0.0005 \mathrm{i}$ \\
$0.9842+0.0006 \mathrm{i}$ & $0.8670+0.0025 \mathrm{i}$ & $-0.0256+0.0062 \mathrm{i}$ & $-0.3098-0.0139 \mathrm{i}$ & $0.0530-0.0077 \mathrm{i}$ \\
$0.8622-0.0003 \mathrm{i}$ & $1.0000+0.0000 \mathrm{i}$ & $0.2119-0.0129 \mathrm{i}$ & $0.7640+0.0596 \mathrm{i}$ & $-0.3595+0.0332 \mathrm{i}$ \\
$0.4555-0.0027 \mathrm{i}$ & $0.5986-0.0111 \mathrm{i}$ & $0.2239-0.0297 \mathrm{i}$ & $1.0000+0.0000 \mathrm{i}$ & $1.0000+0.0000 \mathrm{i}$ \\
\hline \multicolumn{5}{|c|}{ Modos Identificados - Ruido 5\%- $\left(L=L_{\max }\right)$} \\
\hline Modo 1- $\left(\phi_{1}\right)$ & Modo 2- $\left(\phi_{2}\right)$ & Modo 3 $-\left(\phi_{3}\right)$ & Modo 4- $\left(\phi_{4}\right)$ & Modo 5 - $\left(\phi_{5}\right)$ \\
\hline $0.8487+0.0054 \mathrm{i}$ & $-0.5129-0.0042 \mathrm{i}$ & $1.0000+0.000 \mathrm{i}$ & $-0.1070+0.0229 \mathrm{i}$ & $0.0050-0.0014 \mathrm{i}$ \\
$1.0000+0.0000 \mathrm{i}$ & $-0.4460-0.0082 \mathrm{i}$ & $-0.2520+0.0281 \mathrm{i}$ & $0.0558+0.0124 \mathrm{i}$ & $0.0012-0.0006 \mathrm{i}$ \\
$0.9864+0.0011 \mathrm{i}$ & $0.8690+0.0019 \mathrm{i}$ & $-0.0465+0.0281 \mathrm{i}$ & $-0.3068+0.0048 \mathrm{i}$ & $0.0566-0.0087 \mathrm{i}$ \\
$0.8797+0.0057 \mathrm{i}$ & $1.0000+0.0000 \mathrm{i}$ & $0.1949+0.0031 \mathrm{i}$ & $0.7653+0.0628 \mathrm{i}$ & $-0.3543+0.0315 \mathrm{i}$ \\
$0.5227+0.0214 \mathrm{i}$ & $0.6001-0.0122 \mathrm{i}$ & $0.2061-0.0141 \mathrm{i}$ & $1.0000+0.0000 \mathrm{i}$ & $1.0000+0.0000 \mathrm{i}$ \\
\hline
\end{tabular}

Com os erros em relação aos modos, descritos na Tabela 6.19, pode-se tirar a mesma conclusão referente às freqüências naturais e fatores de amortecimento, isto é, que o tamanho da amostra no processo de identificação não influenciou nas estimativas quando utilizou-se dados livres de ruído. Para as simulações onde foi considerada a adição de 5\% de ruído aos dados de saída, os erros não chegaram atingir 5\%, mostrando assim que o procedimento utilizado na identificação teve um boa performance também no caso de sistemas com alta densidade modal.

Tabela 6.19: Erros dos modos identificados - modelo ARMAV - (caso 2)

\begin{tabular}{|c|c|c|c|}
\hline \multicolumn{4}{|c|}{ Erro $(\%)-$ Modos Identificados } \\
\hline $\begin{array}{c}\text { Ruido 0\% } \\
(L=500)\end{array}$ & $\begin{array}{c}\text { Ruido 0\% } \\
\left(L=L_{\max }\right)\end{array}$ & $\begin{array}{c}\text { Ruido 5\% } \\
(L=500)\end{array}$ & $\begin{array}{c}\text { Ruido 5\% } \\
\left(L=L_{\max }\right.\end{array}$ \\
\hline \hline 0.0020 & 0.0020 & 4.824 & 2.636 \\
\hline 0.0005 & 0.0005 & 0.7850 & 0.6007 \\
\hline 0.0016 & 0.0016 & 0.9869 & 1.250 \\
\hline 0.0014 & 0.0014 & 1.522 & 0.0583 \\
\hline \hline 0.0004 & 0.0004 & 0.0235 & 0.4209 \\
\hline
\end{tabular}

A comparação dos modos de vibrar, através da representação geométrica dos auto-vetores normalizados, obtidos das identificações com diferentes tamanhos de amostras, considerando o ruído adicionado ou não aos dados de saída, está apresentada na Figura 6.13. 

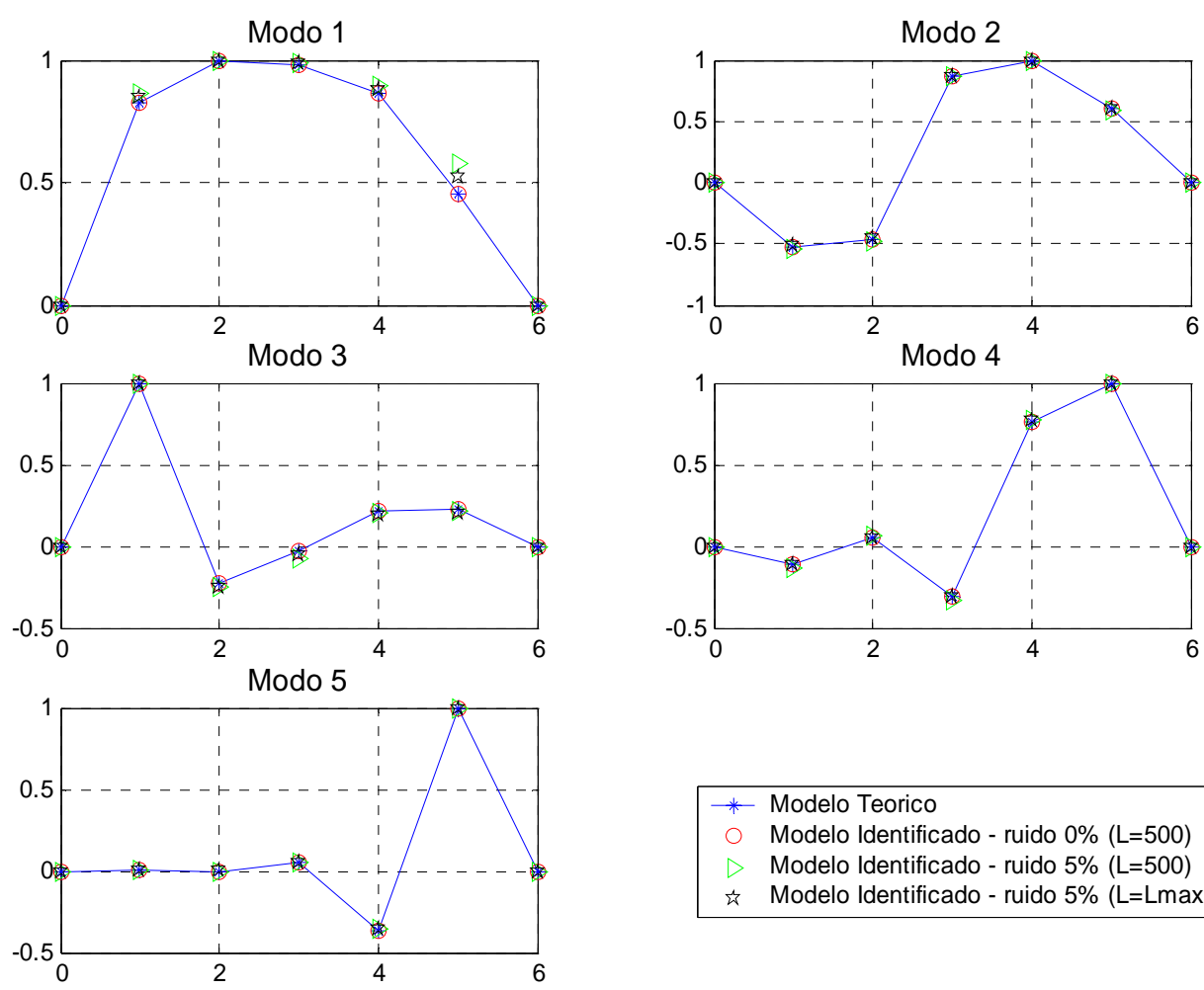

Modelo Teorico

Modelo Identificado - ruido $0 \%(L=500)$ Modelo Identificado - ruido $5 \%(L=500)$ Modelo Identificado - ruido 5\% (L=Lmax)

Figura 6.13: Comparação entre as formas dos modos de vibrar teóricos e identificados modelo ARMAV- (caso 2)

Como pode ser visto na Tabela 6.20, através dos valores assumidos pelo índice MAC, para todas as situações analisadas, pode-se confirmar os resultados satisfatórios na identificação dos parâmetros do sistema com alta densidade modal utilizando o modelo ARMAV.

Tabela 6.20: Valores assumidos por MAC - modelo ARMAV - (caso 2)

\begin{tabular}{|c||c|c|c|}
\hline \multicolumn{4}{|c|}{ Valores - MAC } \\
\hline $\begin{array}{c}\text { Ruido 0\% } \\
(L=500)\end{array}$ & $\begin{array}{c}\text { Ruido 0\% } \\
\left(L=L_{\max }\right)\end{array}$ & $\begin{array}{c}\text { Ruido 5\% } \\
(L=500)\end{array}$ & $\begin{array}{c}\text { Ruido 5\% } \\
\left(L=L_{\max }\right)\end{array}$ \\
\hline \hline 1.0000 & 1.0000 & 0.9962 & 0.9988 \\
\hline \hline 1.0000 & 1.0000 & 0.9997 & 0.9988 \\
\hline \hline 1.0000 & 1.0000 & 0.9869 & 0.9972 \\
\hline \hline 1.0000 & 1.0000 & 0.9953 & 0.9995 \\
\hline \hline 1.0000 & 1.0000 & 1.0000 & 0.9999 \\
\hline
\end{tabular}


$\mathrm{Na}$ Tabela 6.21 está descrito os erros relativos percentuais obtidos quando apenas duas, três e quatro medidas de respostas foram consideradas no processo de identificação.

Tabela 6.21: Erros dos parâmetros modais identificados considerando número reduzido de pontos de medição - modelo ARMAV - (caso 2)

\begin{tabular}{|c|c|c|c|}
\hline \multicolumn{4}{|c|}{ Erro $(\%)$ - Parâmetros Modais $\left(L=L_{\max }\right)$ - Ruído 5\% } \\
\hline \multicolumn{4}{|c|}{4 pontos de medidas de respostas : $m_{1}, m_{2}, m_{3}$ e $m_{4}$} \\
\hline & $\omega_{j}$ & $\zeta_{j}$ & Modos $-\left(\phi_{j}\right)$ \\
\hline \multirow{5}{*}{ ARMAV $(7,7)$} & 0.0027 & 0.1099 & 0.9972 \\
\hline & 0.0008 & 0.0853 & 0.2180 \\
\hline & 0.0001 & 0.0329 & 0.0831 \\
\hline & 0.0005 & 0.4914 & 0.0032 \\
\hline & 0.0003 & 0.0906 & 2.066 \\
\hline \multicolumn{4}{|c|}{3 pontos de medidas de respostas : $m_{1}, m_{2}$ e $m_{3}$} \\
\hline & $\omega_{j}$ & $\zeta_{j}$ & $\operatorname{Modos}-\left(\phi_{j}\right)$ \\
\hline \multirow[t]{5}{*}{ ARMAV $(11,11)$} & 0.0030 & 0.1075 & 0.7326 \\
\hline & 0.0008 & 0.0854 & 1.052 \\
\hline & 0.0001 & 0.0330 & 3.680 \\
\hline & 0.0005 & 0.4915 & 0.4591 \\
\hline & 0.0003 & 0.0906 & 12.24 \\
\hline \multicolumn{4}{|c|}{2 pontos de medidas de respostas : $m_{1}$ e $m_{2}$} \\
\hline & $\omega_{j}$ & $\zeta_{j}$ & $\operatorname{Modos}-\left(\phi_{j}\right)$ \\
\hline \multirow[t]{5}{*}{ ARMAV $(70,70)$} & 0.0035 & 1.038 & 0.3101 \\
\hline & 0.0021 & 0.1936 & 0.4486 \\
\hline & 0.0001 & 0.0366 & 0.1910 \\
\hline & 0.0004 & 0.4881 & 2.595 \\
\hline & 0.0003 & 0.0905 & 52.32 \\
\hline
\end{tabular}

Fazendo uma análise desses resultados, observa-se que a maior parte das estimativas referentes às freqüências naturais e aos fatores de amortecimento não sofreu alterações significativas, de uma identificação para outra, nos casos de duas, três e quatro medidas de respostas utilizadas. Em relação aos modos, as mudanças foram mais acentuadas com erros alcançando quase $13 \%$, no caso da utilização de 3 sinais de saída. Quando apenas duas saídas foram consideradas, o quarto modo alcançou isoladamente um erro de aproximadamente $52.5 \%$. Isso provavelmente ocorreu devido a localização não adequada dos pontos de captação de saída, próximos a nós da estrutura. 
Os valores numéricos assumidos por MAC, mostrados na Tabela 6.22 ,confirma a boa correlação da forma entre os modos teóricos e identificados, exceto para o quinto modo, quando apenas duas medidas de respostas foram consideradas no procedimento de identificação.

Tabela 6.22: Valores assumidos por MAC - Modelo ARMAV - (caso 2) considerando número reduzido de pontos de medição

\begin{tabular}{|c|c|c|}
\hline \multicolumn{3}{|c|}{ Valores MAC - Ruido $5 \%\left(L=L_{\max }\right)$} \\
\hline \hline $\begin{array}{c}\text { 4 pontos de medidas de } \\
\text { respostas: } m_{1}, m_{2}, m_{3} e \\
m_{4}\end{array}$ & $\begin{array}{c}\text { 3 pontos de medidas de } \\
\text { respostas: } m_{1}, m_{2} \text { e } m_{3}\end{array}$ & $\begin{array}{c}\text { 2 pontos de medidas } \\
\text { de respostas: } m_{1} \text { e } m_{2}\end{array}$ \\
\hline 0.9999 & 0.9999 & 1.0000 \\
\hline 0.9997 & 0.9994 & 1.0000 \\
\hline 0.9975 & 0.9979 & 0.9997 \\
\hline 0.9991 & 0.9974 & 0.9776 \\
\hline 0.9996 & 0.9875 & 0.2306 \\
\hline
\end{tabular}

Realizando uma análise comparativa entre os dados da Tabela 6.21 e aqueles obtidos na identificação quando foram utilizadas as cinco saídas do sistema, nas mesmas condições, verifica-se algo aparentemente inesperado, principalmente relacionado com o primeiro modo. Observa-se que as freqüências naturais estimadas com seus respectivos fatores de amortecimento e modos de vibrar, identificados através do modelo ARMAV $(3,3)$ utilizando todas as saídas, apresentam erros muito maiores quando comparadas com aqueles apresentados na Tabela 6.21. Isso se deve ao fato de que o modelo ARMAV utilizado deveria ter sido de ordem mínima $p=4$, como pode ser vista através do diagrama ilustrado na Figura $6.11(\mathrm{~d})$, e não $p=3$ como foi estabelecida pelo critério BIC.

Além disso, a ordem do modelo $\operatorname{ARMAV}(70,70)$, utilizado na identificação para o caso onde somente duas medidas de respostas foram consideradas, foi encontrada através do auxílio do diagrama de estabilidade. Utilizando o critério BIC a ordem seria de $p=37$, tornando impossível a identificação das duas freqüências mais baixas do sistema. 
Para efeito de ilustração, será mostrado o teste de validação do modelo $\operatorname{ARMAV}(3,3)$ obtido a partir das cinco medidas de respostas contaminadas por ruído, com $500+p$ pontos. A Figura 6.14 ilustra o resultado do teste através da função de autocorrelação (a) e do histograma (b), do sinal residual correspondente à saída 1 , garantindo que o sinal se aproxima de um ruído branco gaussiano. Uma vez que as mesmas conclusões são válidas também para as saídas 2, 3 e 4 e 5, pode-se afirmar que o modelo $\operatorname{ARMAV}(3,3)$ se ajusta bem aos dados pelos quais ele foi estimado.

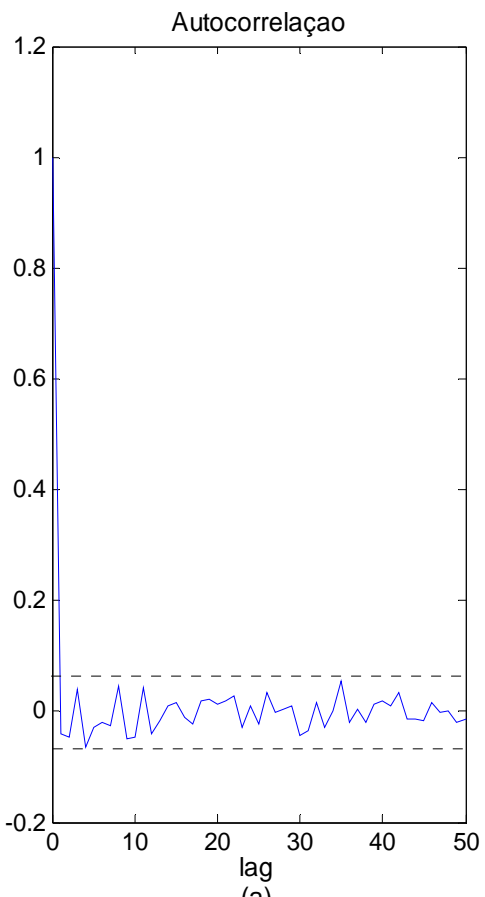

(a)

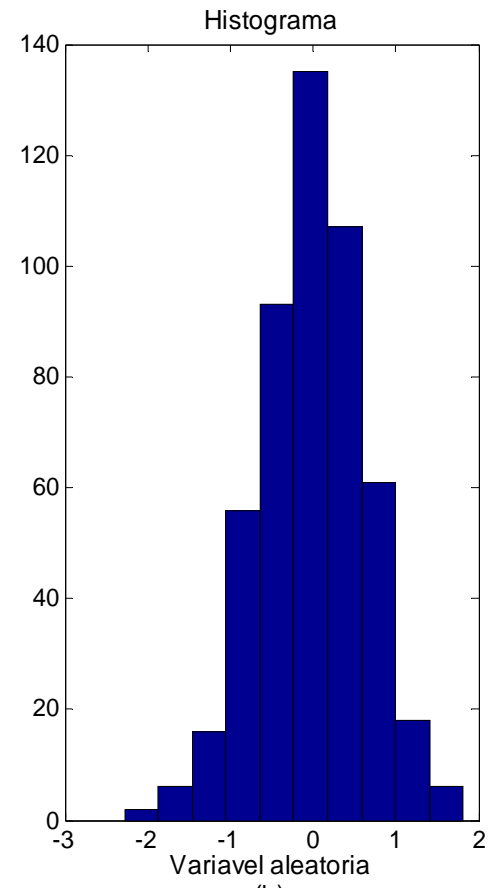

(b)

Figura 6.14: Teste do sinal residual da saída 1:

(a) função de auto-correlação; (b) histograma - (caso 2)

A identificação realizada através do modelo $\mathrm{ARV}$, para vários valores de $p$ pertencentes ao intervalo [2, 40], fornece as estimativas das freqüências, como podem ser visualizadas graficamente através do diagrama representado na Figura 6.15. Esses resultados foram obtidos utilizando no processo de identificação, as cinco medidas de respostas, livres e corrompidas por ruído, e considerando uma amostra com 80.000 pontos discretizados em intervalos de $\Delta t=0.0002$ segundos. A partir desses diagramas de estabilidade torna-se evidente que o sistema analisado possui cinco freqüências naturais na banda de $[0,30] \mathrm{Hz}$, sendo $30 \mathrm{~Hz}$ a freqüência de corte pré-estabelecida. 


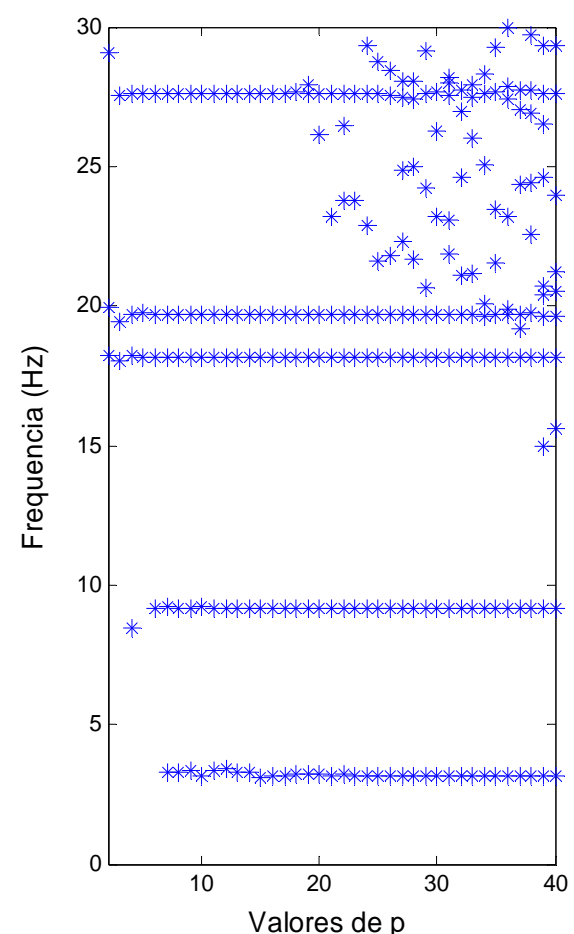

(a)

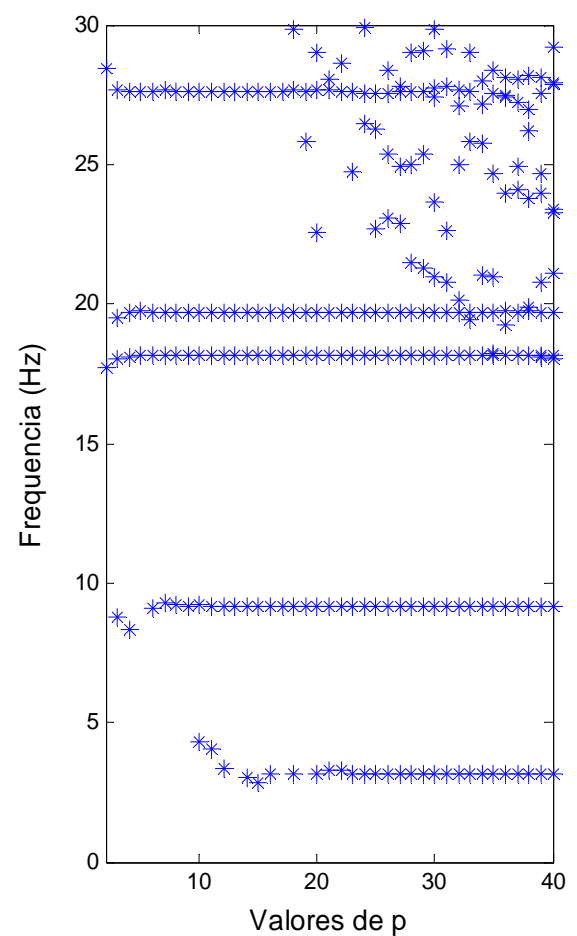

(b)

Figura 6.15: Diagramas de estabilidade - modelo ARV-(caso 2)

(a) ruído $0 \%$; (b) ruído $5 \%$

A partir das freqüências identificadas, o conjunto final que representa os parâmetros modais foi obtido segundo o algoritmo descrito na Figura 6.3. Para este caso foram considerados os mesmos valores de $\varepsilon, \varepsilon_{1}$ e $\varepsilon_{2}$ necessários no processo de seleção da melhor estimativa, utilizados no exemplo anterior. O resultado dessa seleção encontra-se nas Tabelas 6.23 e 6.24, e corresponde às estimativas das freqüências naturais, fatores de amortecimento e modos de vibrar do sistema estrutural, com os respectivos valores $p_{j}$. Cada $p_{j}$ representa a ordem do modelo pelo qual os parâmetros foram encontrados.

Tabela 6.23: Parâmetros modais identificados - modelo ARV - (caso 2)

\begin{tabular}{|c|c|c|c||c||}
\hline \multicolumn{5}{|c||}{ Ruído-0\%-ARV $\left(p_{j}\right)$} \\
\hline \hline$p_{j}$ & $\omega_{j}(\mathrm{~Hz})$ & Erro $(\%)$ & $\zeta_{j}$ & Erro $(\%)$ \\
\hline 23 & 3.188 & 0.9795 & 0.0068 & 0.0537 \\
\hline \hline 23 & 9.182 & 0.5650 & 0.0092 & 10.93 \\
\hline \hline 21 & 18.14 & 0.6647 & 0.0116 & 5.732 \\
\hline \hline 22 & 19.68 & 0.7468 & 0.0184 & 2.886 \\
\hline \hline 21 & 27.60 & 0.6892 & 0.0344 & 2.378 \\
\hline
\end{tabular}


Tabela 6.23: Parâmetros modais identificados - modelo ARV - (caso 2) - (continuação)

\begin{tabular}{|c||c|c|c|c||}
\hline \multicolumn{5}{|c||}{ Ruido-5\% $-\operatorname{ARV}\left(p_{j}\right)$} \\
\hline$p_{j}$ & $\omega_{j}(\mathrm{~Hz})$ & Erro $(\%)$ & $\zeta_{j}$ & Erro $(\%)$ \\
\hline 28 & 3.181 & 0.7798 & 0.0079 & 15.90 \\
\hline \hline 23 & 9.178 & 0.5216 & 0.0089 & 5.521 \\
\hline 23 & 18.14 & 0.6617 & 0.0117 & 5.888 \\
\hline 22 & 19.70 & 0.8251 & 0.0184 & 3.347 \\
\hline \hline 21 & 27.64 & 0.8476 & 0.0317 & 5.512 \\
\hline
\end{tabular}

Tabela 6.24: Modos de vibrar identificados - modelo ARV - (caso 2)

\begin{tabular}{|c|c|c|c|c|}
\hline \multicolumn{5}{|c|}{ Modos Identificados - Ruído 0\% - ARV $\left(p_{j}\right)$} \\
\hline Modo $1-\left(\phi_{1}\right)$ & Modo $2-\left(\phi_{2}\right)$ & Modo $3-\left(\phi_{3}\right)$ & Modo $4-\left(\phi_{4}\right)$ & Modo $5-\left(\phi_{5}\right)$ \\
\hline $0.8339-0.0013 \mathrm{i}$ & $-0.5263+0.0039 i$ & $1.0000+0.0000 \mathrm{i}$ & & $0.0047-0.0010 \mathrm{i}$ \\
\hline & & & & \\
\hline & & & & \\
\hline & & & & \\
\hline & $0.6012-0.0056 \mathrm{i}$ & $0.2703-0.0852 \mathrm{i}$ & $1.0000+0.0000 \mathrm{i}$ & \\
\hline \multicolumn{5}{|c|}{ Modos Identificados - Ruido 5\% - ARV $\left(p_{j}\right)$} \\
\hline Modo $1-\left(\phi_{1}\right)$ & Modo $2-\left(\phi_{2}\right)$ & Modo $3-\left(\phi_{3}\right)$ & Modo $4-\left(\phi_{4}\right)$ & Modo $5-\left(\phi_{5}\right)$ \\
\hline & & & & $-0.0012-0.0005 i$ \\
\hline $1.0000+0.0000 \mathrm{i}$ & -0.4562 & $-0.2270+0.003$ & $-0.0042 i$ & $-0.0025+0.0007 i$ \\
\hline $0.9859-0.0068 \mathrm{i}$ & $0.8652+0.0142 \mathrm{i}$ & $-0.0355+0.0257 \mathrm{i}$ & $-0.3047+0.0027 i$ & $0.0493-0.0087 i$ \\
\hline $0.8668+0.0091 \mathrm{i}$ & $1.0000+0.0000 \mathrm{i}$ & $0.2401-0.0526 \mathrm{i}$ & $0.7618+0.0581 \mathrm{i}$ & $-0.3413+0.0318 \mathrm{i}$ \\
\hline $0.4605+0.0033 \mathrm{i}$ & $0.6024-0.0060 \mathrm{i}$ & $0.2602-0.0831 \mathrm{i}$ & $1.0000+0.0000 \mathrm{i}$ & $1.0000+0.0000 \mathrm{i}$ \\
\hline
\end{tabular}

Através dos valores apresentados nas Tabelas 6.23 e 6.25 observa-se que os erros em relação às freqüências, fatores de amortecimento e modos de vibrar não ultrapassaram $1 \%, 16 \%$ e $7 \%$, respectivamente. Isso mostrou que a técnica utilizada também identificou de maneira satisfatória o sistema que possui alta densidade modal.

Tabela 6.25: Erros dos modos identificados -modelo ARV - (caso 2)

\begin{tabular}{|c|c|}
\hline \multicolumn{2}{|c|}{ Erro (\%) - Modos Identificados } \\
\hline Ruido 0\% & Ruido 5\% \\
\hline 0.0557 & 0.2608 \\
\hline 0.0088 & 0.1153 \\
\hline 6.8544 & 5.7079 \\
\hline 0.5223 & 0.3762 \\
\hline 0.4359 & 0.7110 \\
\hline \hline
\end{tabular}


A confirmação da identificação satisfatória dos parâmetros modais pode ser constatada também através dos valores do índice MAC apresentados na Tabela 6.26.

Tabela 6.26: Valores assumidos por MAC - modelo ARV - (caso 2)

\begin{tabular}{|c|c|}
\hline \multicolumn{2}{|c|}{ Valores - MAC } \\
\hline Ruido 0\% & Ruido 5\% \\
\hline 1.0000 & 1.0000 \\
\hline 0.9999 & 0.9999 \\
\hline 0.9934 & 0.9945 \\
\hline 0.9999 & 0.9999 \\
\hline 0.9999 & 0.9997 \\
\hline
\end{tabular}

A Figura 6.16 mostra uma comparação gráfica da forma dos modos entre o modelo teórico e os modelos identificados a partir dos dados livre e corrompido por ruído.
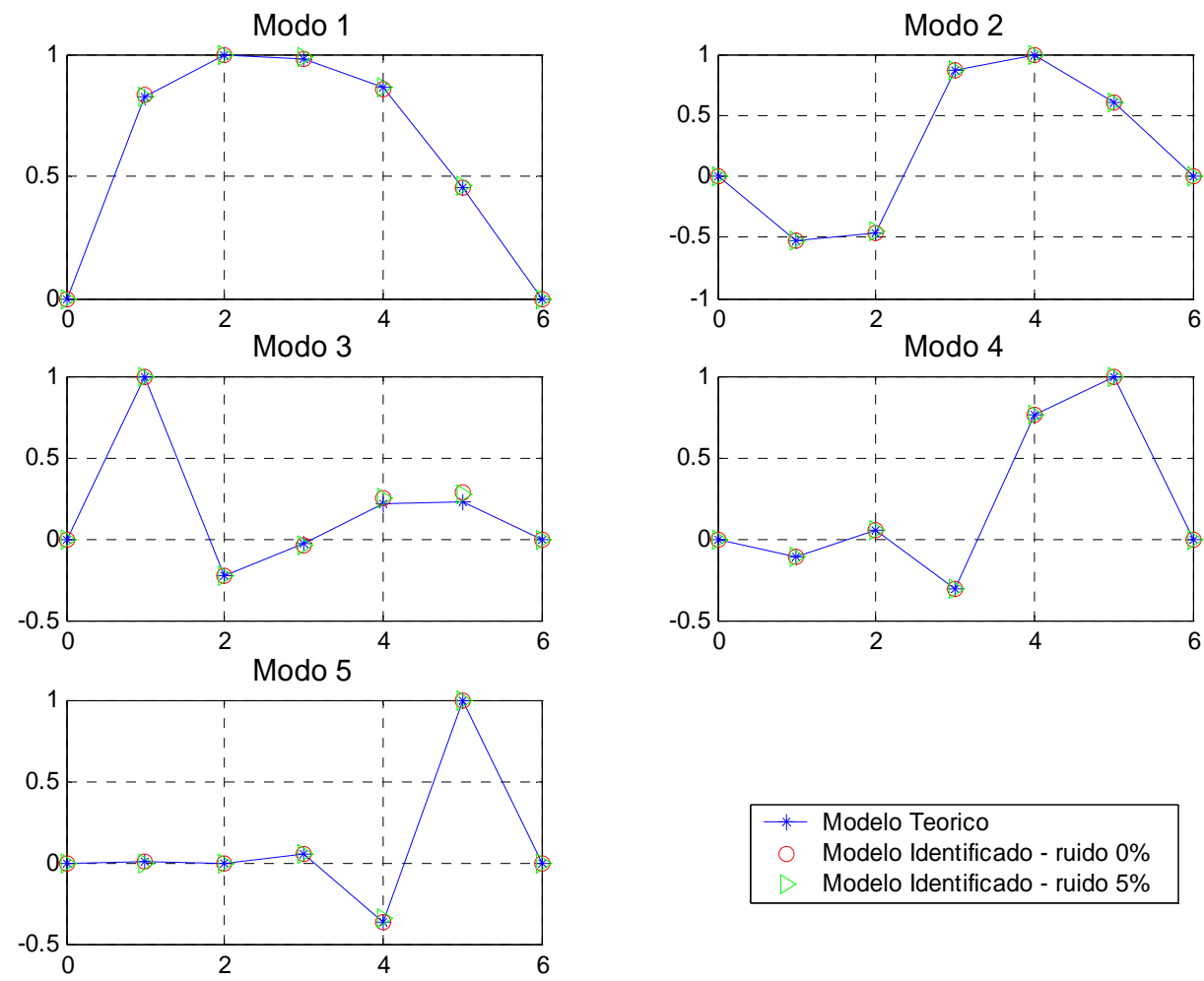

Figura 6.16: Comparação entre as formas dos modos de vibrar teóricos e identificados - modelo ARV - (caso 2) 
Analisando os dados da Tabela 6.27, nota-se que para os casos onde apenas 3 e 4 saídas foram consideradas, todas as freqüências naturais do sistema foram identificadas na faixa de $\left[\begin{array}{ll}0 & 30\end{array}\right] \mathrm{Hz}$. Nestes casos, os intervalos de $p$ "escolhidos" necessários para realizar as identificações foram de [2 50] e [2 60], respectivamente.

Tabela 6.27: Erros dos parâmetros modais identificados considerando número reduzido de pontos de medição - modelo ARV - (caso 2)

\begin{tabular}{|c|c|c|c|}
\hline \multicolumn{4}{|c|}{ Erro(\%) - Parâmetros Modais-Ruído 5\% } \\
\hline \multicolumn{4}{|c|}{4 pontos de medidas de respostas: $m_{1}, m_{2}, m_{3}$ e $m_{4}$} \\
\hline$p_{j}$ & $\omega_{j}$ & $\zeta_{j}$ & $\phi_{j}$ \\
\hline 28 & 0.5859 & 4.077 & 0.0713 \\
\hline 25 & 0.5604 & 11.67 & 0.2439 \\
\hline 22 & 0.6704 & 6.305 & 3.164 \\
\hline 25 & 0.7541 & 3.898 & 0.7155 \\
\hline 23 & 0.9004 & 0.016 & 0.0841 \\
\hline \multicolumn{4}{|c|}{3 pontos de medidas de respostas : $m_{1}, m_{2}$ e $m_{3}$} \\
\hline 36 & 0.5165 & 1.855 & 0.0477 \\
\hline 33 & 0.5280 & 8.461 & 0.1982 \\
\hline 26 & 0.6697 & 6.932 & 1.923 \\
\hline 34 & 0.7069 & 12.61 & 2.395 \\
\hline 35 & 0.9337 & 13.45 & 0.8729 \\
\hline \multicolumn{4}{|c|}{2 pontos de medidas de respostas : $m_{1}$ e $m_{2}$} \\
\hline 61 & 0.5939 & 7.057 & 0.0165 \\
\hline 60 & 0.5492 & 6.205 & 0.0747 \\
\hline 62 & 0.9839 & 5.530 & 0.0379 \\
\hline 65 & 0.3935 & 14.43 & 0.0586 \\
\hline- & - & - & - \\
\hline
\end{tabular}

Para a identificação realizada somente com 2 medidas de respostas houve a perda da freqüência mais alta do sistema. A ocorrência deste fato, possivelmente se deu pela escolha de pontos de saídas próximos de nós da estrutura. Neste caso a identificação foi realizada considerado um intervalo de variação de $p$ igual a [40 80].

No entanto, pode-se dizer que em geral, os resultados obtidos quando apenas algumas saídas foram consideradas no processo de identificação foram satisfatórios, uma vez que os erros em relação às freqüências, fatores de amortecimento e modos de vibrar foram inferiores a $1 \%, 15 \%$ e $3.5 \%$, respectivamente. 
A boa qualidade dos parâmetros modais identificados pode também ser verificada através dos valores assumidos pelo índice MAC, apresentados na Tabela 6.28 .

Tabela 6.28: Valores assumidos por MAC - Modelo ARV - (caso 2) considerando número reduzido de pontos de medição

\begin{tabular}{|c|c|c|}
\hline \multicolumn{3}{|c|}{ Valores MAC - Ruido 5\% } \\
\hline $\begin{array}{c}\text { 4 pontos de medidas de } \\
\text { respostas: } m_{1}, m_{2}, m_{3} e \\
m_{4}\end{array}$ & $\begin{array}{c}\text { 3 pontos de medidas de } \\
\text { respostas: } m_{1}, m_{2} \text { e } m_{3}\end{array}$ & $\begin{array}{c}\text { 2 pontos de medidas de } \\
\text { respostas: } m_{1} \text { e } m_{2}\end{array}$ \\
\hline 1.0000 & 1.0000 & 1.0000 \\
\hline 0.9999 & 0.9999 & 0.9999 \\
\hline 0.9972 & 0.9994 & 1.0000 \\
\hline 0.9998 & 0.9989 & 0.9991 \\
\hline \hline 1.0000 & 0.9988 & - \\
\hline \hline
\end{tabular}

É importante destacar que, para os dois modelos diferentes analisados aqui neste trabalho, foi possível realizar o processo de identificação em uma única etapa, sem a necessidade de fazer a identificação por faixas de freqüências. 


\section{Aplicação Experimental}

\subsection{PRELIMINARES}

Este capítulo tem por objetivo avaliar o desempenho dos métodos que utilizam os modelos ARMAV e ARV, representados pelos algoritmos 6.1 e 6.2 apresentados no capítulo anterior, na identificação dos parâmetros modais a partir de dados experimentais. Para tanto será descrito o ensaio experimental realizado em uma placa de alumínio, e apresentados os procedimentos e equipamentos utilizados na aquisição dos dados experimentais. Em seguida os resultados obtidos nos procedimentos de identificação serão apresentados e analisados.

\subsection{DESCRIÇÃO DO MODELO EXPERIMENTAL}

A estrutura submetida à análise modal experimental, para verificar 0 desempenho dos métodos de identificação apresentados, é uma placa de alumínio cujas dimensões da área livre da placa são de $360 \mathrm{~mm}$ de comprimento e largura, e espessura igual a $3 \mathrm{~mm}$. Esta estrutura foi amplamente estudada em alguns trabalhos desenvolvidos por pesquisadores do departamento de engenharia mecânica na Escola de Engenharia de São Carlos (EESC). Dentre os quais pode-se citar: TSUNAKI (1999), PAZIANI (2002) e REBOLHO (2006). Assim, o ensaio experimental realizado neste 
trabalho se beneficia do conhecimento das características da placa de alumínio utilizada, proporcionado pelos trabalhos realizados anteriormente.

Para realizar o ensaio experimental a placa foi engastada em um dos seus lados entre duas chapas de aço espessas, através de parafusos, e em seguida, todo o conjunto foi fixado à uma base inercial, conforme mostra a Figura 7.1

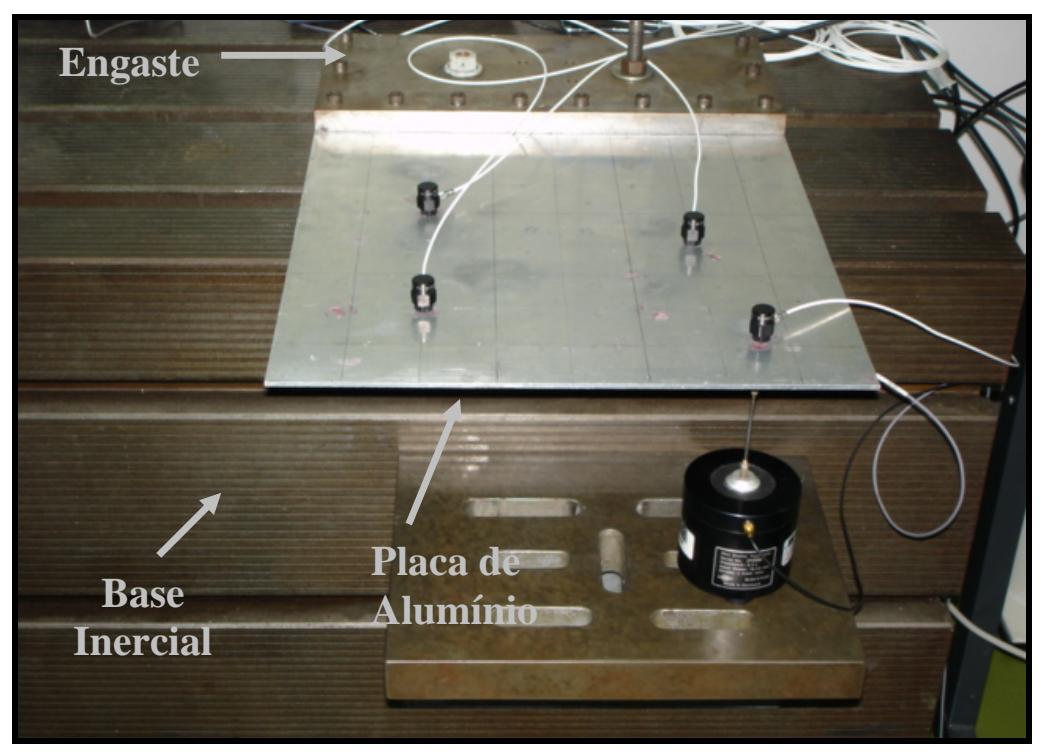

Figura 7.1: Placa de alumínio utilizada no ensaio experimental

O procedimento de identificação foi desenvolvido com base na análise de sinais SIMO. No processo de identificação foram estabelecidos cinco pontos de coleta de dados, sendo um ponto de entrada (força de excitação) e quatro pontos de saída (acelerações desses pontos). Foram realizados dois ensaios considerando as mesmas localizações para os pontos de saída e duas localizações distintas $\boldsymbol{A}$ e $\boldsymbol{B}$ para os pontos de excitação (ver figura 7.2).

A localização das excitações está diretamente ligada ao grau de controlabilidade do sistema e a localização dos pontos de saída está diretamente ligada ao grau de observabilidade do sistema. O conjunto total das FRFs traz informações sobre os graus de observabilidade e de controlabilidade, relativas às localizações das saídas e entradas do sistema. No entanto, deve-se levar em conta que limitações ocasionadas por procedimentos experimentais, tais como a resolução das FRFs e o nível de ruído presente nos dados experimentais, também afetam os graus de observabilidade e controlabilidade contidos nas FRFs (TSUNAKI, 1999). 
Nos ensaios experimentais realizados neste trabalho, os dois pontos de entrada, foram localizados de maneira que todos os modos fossem excitados, na faixa operacional de freqüência. Já a localização dos pontos de saída foi escolhida de maneira que todos os modos fossem observados, ou seja, medidos na faixa de freqüência de interesse. Foram escolhidos apenas quatro pontos de saída, devido a disponibilidade de equipamentos de medida no laboratório, no caso os acelerômetros, uma vez que os dados devem ser colhidos e processados simultaneamente.

Neste contexto, as localizações dos pontos excitação e resposta foram determinadas a partir de vários ensaios realizados para diferentes localizações de pontos na placa, através da análise das (FRFs) obtidas através do analisador de sinais SignalCalc ACE. A Figura 7.2 apresenta uma visualização da disposição dos pontos de entrada e saída e da dimensão da placa.

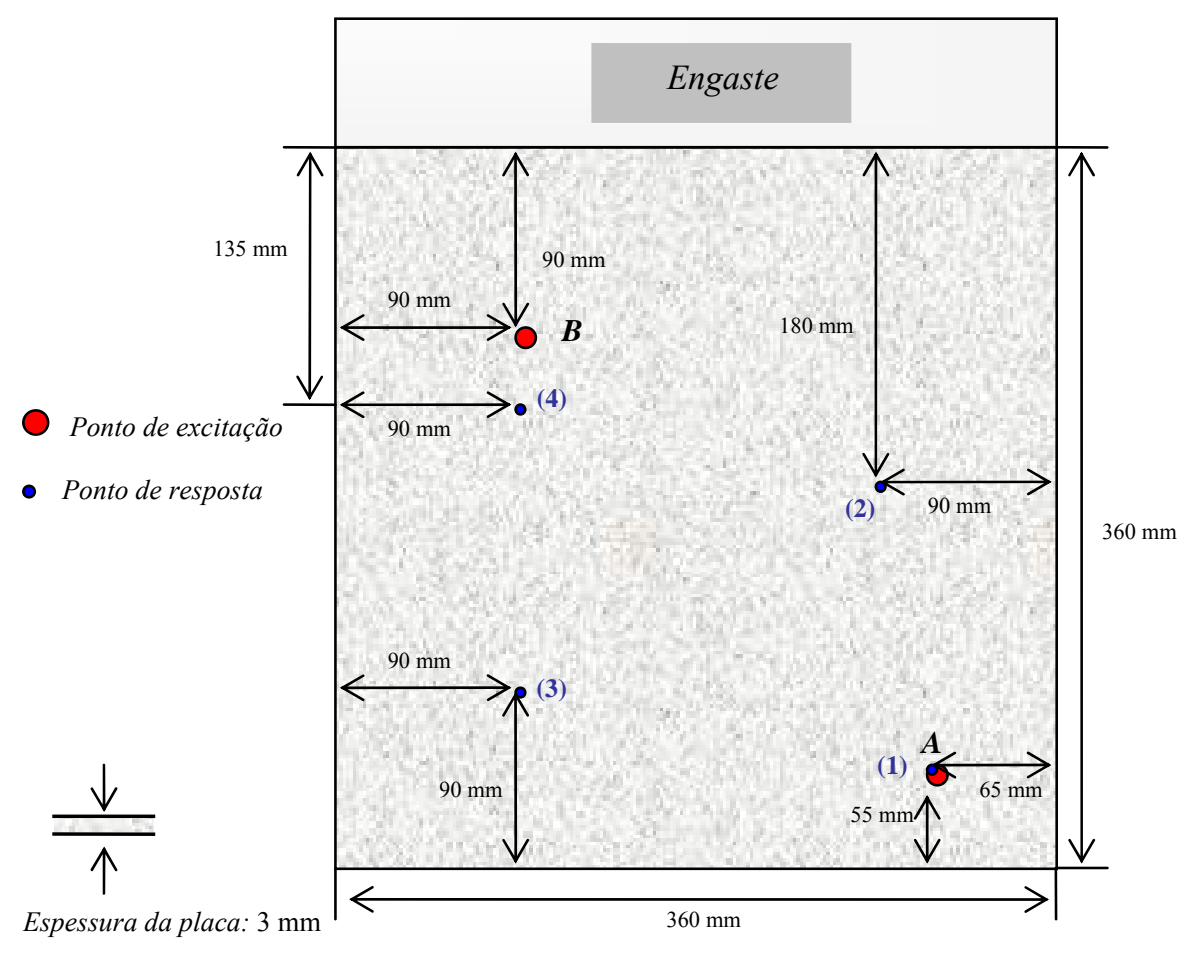

Figura 7.2: Descrição da placa de alumínio e localização dos pontos de entrada e saída

\subsection{AQUISIÇÃO E PROCESSAMENTO DOS SINAIS}

Os ensaios experimentais foram realizados com o objetivo de obter os dados de entrada e saída no domínio do tempo. A identificação dos parâmetros modais a partir do algoritmo que utiliza o modelo ARMAV foi realizada com base nas medidas dos 
sinais de excitação e resposta da estrutura em estudo, enquanto que para o algoritmo de identificação a partir do modelo ARV apenas as medidas de respostas foram utilizadas.

Além da localização adequada dos pontos de excitação e resposta, o tipo de excitação também é um fator importante para o êxito da análise modal experimental.

Os ensaios foram realizados aplicando-se um sinal de entrada aleatório, do tipo ruído branco, escolhido de forma a excitar de maneira uniforme os modos dentro da faixa de freqüência de interesse limitada entre 0 e $250 \mathrm{~Hz}$. Estes sinais gerados pelo analisador de sinais SignalCalc ACE (1) foram amplificados a partir de um amplificador de potência B\&K modelo 2706 (2), e posteriormente enviados para o excitador eletrodinâmico da B\&K 4810 (3). O sinal de excitação foi medido através de um transdutor de força B\&K modelo 8230 (4), posicionado entre o excitador e a placa de alumínio.

Como saídas, foram tomadas as acelerações medidas simultaneamente em quatro pontos, através de acelerômetros piezoelétricos Kistler modelo $8636 \mathrm{C} 10$ (5). Estes acelerômetros foram fixados na placa de alumínio através de uma fina camada de cera apropriada da Kistler. Para cada um dos quatro pontos de reposta, onde foram fixados os acelerômetros, foi tomada a média de dez FRFs afim de minimizar o ruído presente nos sinais. Este recurso é conhecido como averaging. Além disso, foi empregada uma janela Hanning nos sinais de força e aceleração.

Os sinais provenientes do transdutor de força e dos acelerômetros foram enviados para o condicionador e amplificador de sinais Nexus da B\&K modelo 2693A OI4 Deltatron (6) e para o amplificador de carga da Kistler Power Suply Coupler modelo 5134 (7) respectivamente, e posteriormente enviados ao sistema de aquisição e processamento de sinais dSPACE® (8). Este sistema possui uma placa multiprocessadora DS1005 munida de um processador PowerPc de $400 \mathrm{Mhz}$ e 128 Mbytes de memória RAM. A placa processadora DS2003 possui 32 canais de entrada A/D e a placa DS2102 6 canais de saída D/A, de 16 bits de resolução. No entanto, nesse trabalho o sistema dSPACE® foi utilizado somente para realizar a aquisição dos sinais no domínio do tempo. Por fim os dados coletados pela dSPACE® foram transmitidos a um microcomputador (9), visualizados através do software de interface gráfica da dSPACE, denominado ControlDesk ${ }^{\circledR}$ versão 2.5.5, e transformados para o formato requerido, para que pudessem ser utilizados como dados de entrada para o algoritmo de identificação de sistemas. A Figura 7.3 mostra uma foto do aparato instrumental utilizado no procedimento experimental. 


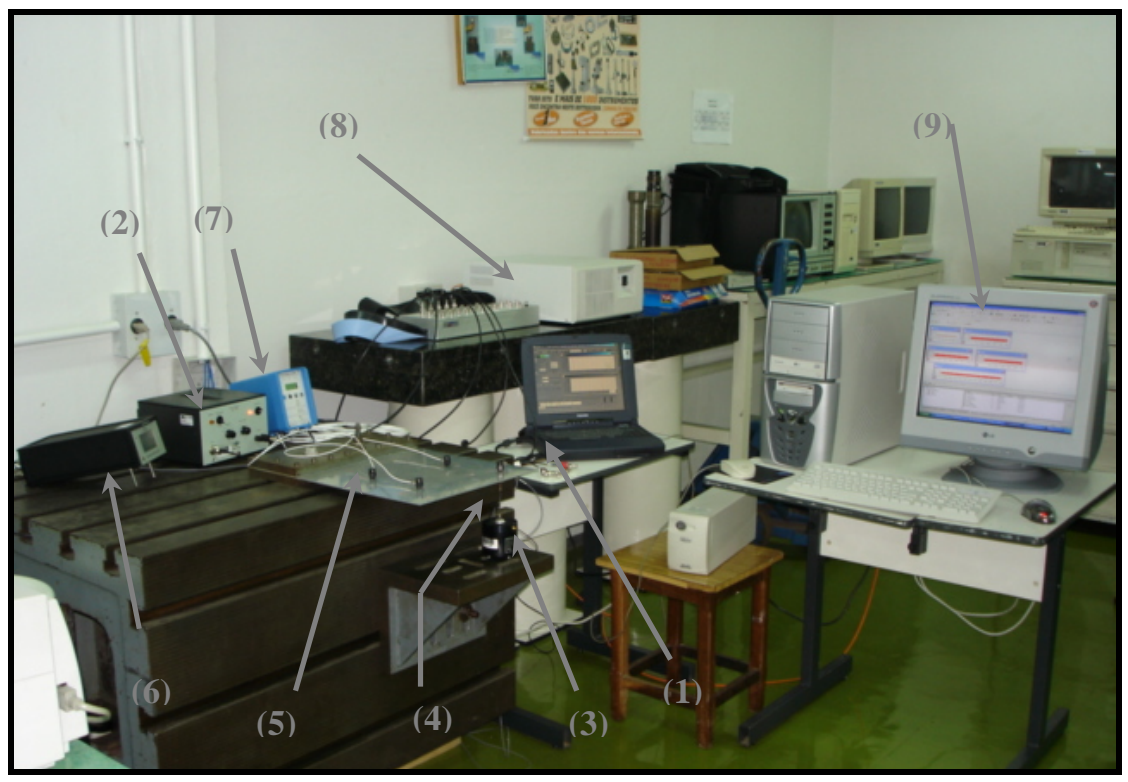

Figura 7.3: Aparato experimental

A relação e descrição dos equipamentos usados no experimento são apresentadas na Tabela 7.1.

Tabela 7.1: Equipamentos utilizados no experimento

\begin{tabular}{|c|c|}
\hline Relação de Equipamentos & \\
\hline Analisador de Sinais de Quatro Canais -SignalCalc ACE & (1) \\
\hline Amplificador de Potência B\&K - Modelo 2706 & (2) \\
\hline Excitador B\&K - Modelo 4810 & (3) \\
\hline $\begin{array}{l}\text { Transdutor de Força B\&K - Modelo } 8230 \\
\text { Sensibilidade }=115.0 \mathrm{mV} / \mathrm{N} \text {; Massa: } 30.2 \mathrm{~g}\end{array}$ & (4) \\
\hline $\begin{array}{l}\text { Acelerômetros Kistler }- \text { Modelo 8636C10; Massa: } 5.0 \mathrm{~g} \\
\text { Sensiblidades }=513 \mathrm{mV} / \mathrm{g}(\mathrm{C} 142674) ; 506 \mathrm{mV} / \mathrm{g}(\mathrm{C} 142675) ; \\
512 \mathrm{mV} / \mathrm{g}(\mathrm{C} 142676) ; 504 \mathrm{mV} / \mathrm{g}(\mathrm{C} 142677) ;\end{array}$ & (5) \\
\hline Condicionador e Amplificador de Sinais B\&K - Nexus modelo 2693A OI4 Deltatron & (6) \\
\hline Amplificador de Sinal Kistler Power Suply Coupler 5134 & (7) \\
\hline Sistema dSPACE® & (8) \\
\hline Microcomputador & (9) \\
\hline
\end{tabular}


O diagrama do aparato experimental utilizado durante o ensaio modal da placa de alumínio, para aquisição dos dados no domínio do tempo utilizando os equipamentos descritos na Tabela 7.1 é ilustrado na Figura 7.4.

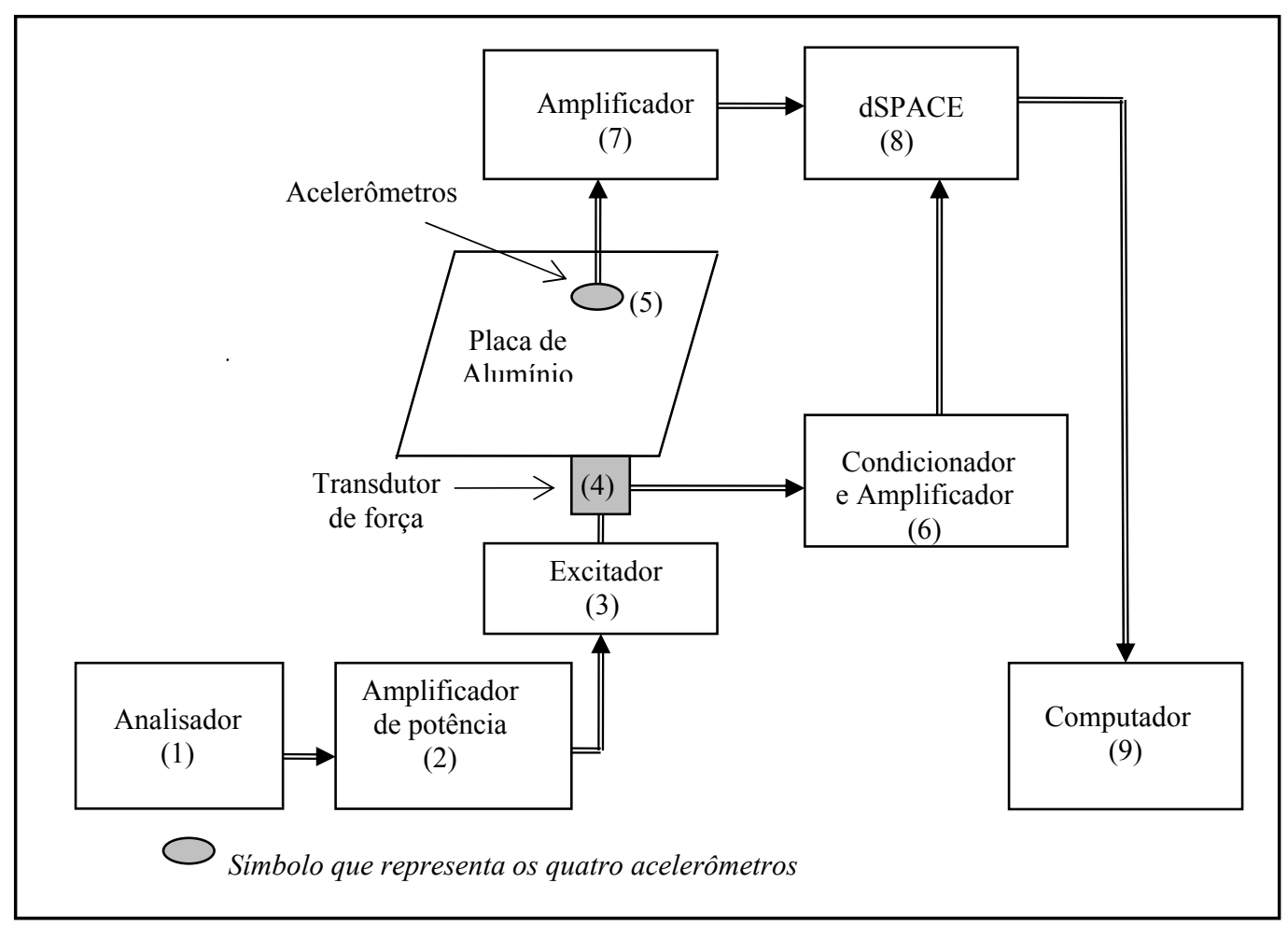

Figura 7.4: Esquema do aparato experimental

Para realizar uma pré-análise do modelo em estudo, a partir das FRFs obtidas através dos sinais do transdutor de força e de cada um dos quatro acelerômetros nas posições escolhidas, calcula-se o espectro de potência médio (EPM) através da seguinte equação:

$$
\operatorname{EPM}\left(\omega_{j}\right)=\frac{\sum_{i=1}^{N}\left|H_{i}\left(\omega_{j}\right)\right|^{2}}{N}
$$

onde $H_{i}\left(\omega_{j}\right)$ é o valor da $i$-ésima FRF na freqüência $\omega_{j}$ e $N$ é o número total de FRFs.

A Figura 7.5 mostra os gráficos dos dois espectros de potência médio obtidos a partir das 4 FRFs, na faixa de freqüência de 0 a $250 \mathrm{~Hz}$, considerando a localização do ponto de excitação $\boldsymbol{A}$ e $\boldsymbol{B}$, respectivamente. Os picos existentes no EPM representam as 
freqüências naturais de cada modo e a sua amplitude relativa. Através do número de picos presentes, pode-se tirar uma informação importante sobre a ordem estimada do sistema, para a faixa de freqüência estabelecida. Como pode ser observada, neste caso a ordem estimada é $2 n=10$, ou seja, há 5 modos, uma vez que existem 5 picos no EPM.

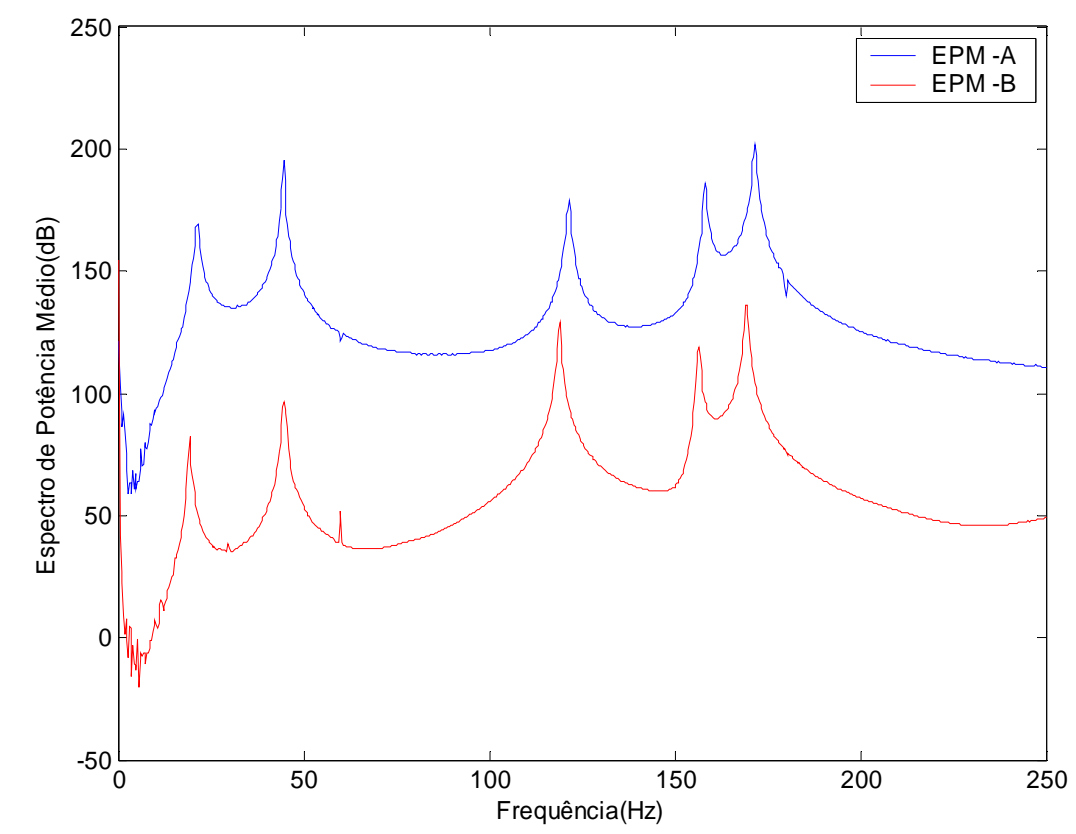

Figura 7.5: Espectro de potência médio - ponto de excitação $\boldsymbol{A}$ e $\boldsymbol{B}$

Comparando os dois gráficos do $E P M$, considerando a excitação no ponto $\boldsymbol{A}$ e no ponto $\boldsymbol{B}$, torna-se possível o conhecimento das amplitudes com que cada modo é excitado em função da localização do ponto de excitação.

\subsection{RESULTADOS}

Os resultados obtidos na identificação realizada a partir dos algoritmos com base nos modelos ARMAV e ARV, descritos na seção 6.2 deste trabalho, serão apresentados e analisados nesta seção. Além disso, para fins de comparação serão fornecidos os valores das cinco primeiras freqüências naturais obtidas da análise de elementos finitos realizado por Tsunaki (1999), sobre a mesma estrutura.

A taxa de aquisição utilizada foi de 0.001 segundos considerando um tempo total de 120 segundos. No entanto, não foi necessária a utilização dos 120.000 pontos no processo de identificação. Para a identificação realizada a partir do modelo ARMAV apenas 1.000 pontos foram utilizados, enquanto que para o caso da identificação através 
do modelo ARV, foram empregados 50.000 pontos. Em ambos algoritmos de identificação, a freqüência de corte foi estipulada em $200 \mathrm{~Hz}$.

Conforme mostra a Figura 7.2, foram realizados dois ensaios na placa de alumínio de acordo com a localização do ponto de excitação. O ensaio cuja excitação está localizada no ponto $\boldsymbol{A}$ será chamado de caso $A$, e no ponto $\boldsymbol{B}$ de caso $B$. Em ambos os casos, os quatro pontos de saída onde foram fixados os acelerômetros, foram mantidos nas mesmas posições.

A Figura 7.6 exibe como exemplo, o sinal de excitação aleatória medido pelo transdutor de força localizado no ponto $\boldsymbol{A}$ e a Figura 7.7 mostra o sinal de resposta medido pelo acelerômetro (1) referente ao ensaio no caso A. Ambos nos 10 primeiros segundos de aquisição.

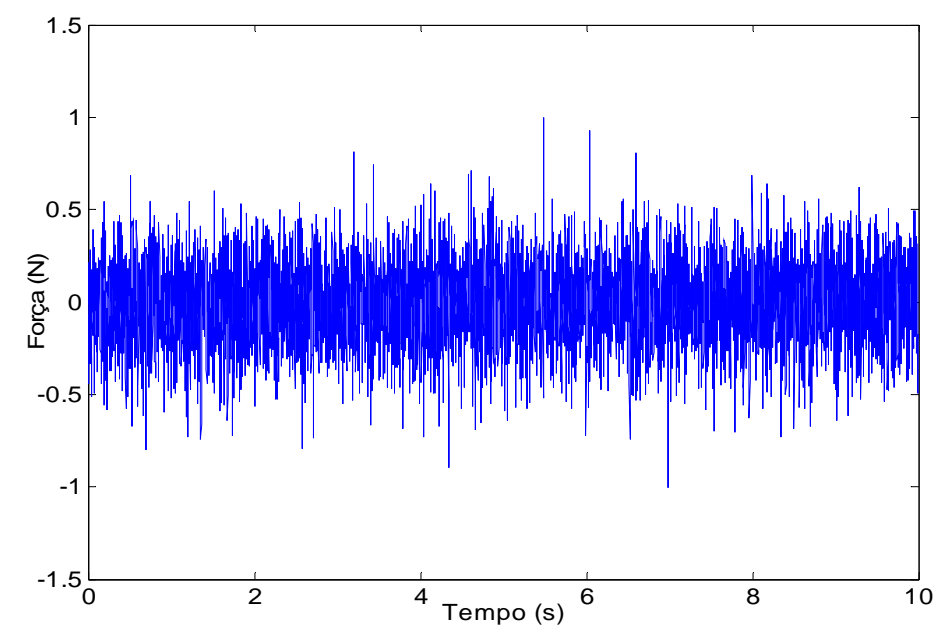

Figura 7.6: Sinal de excitação - caso $A$

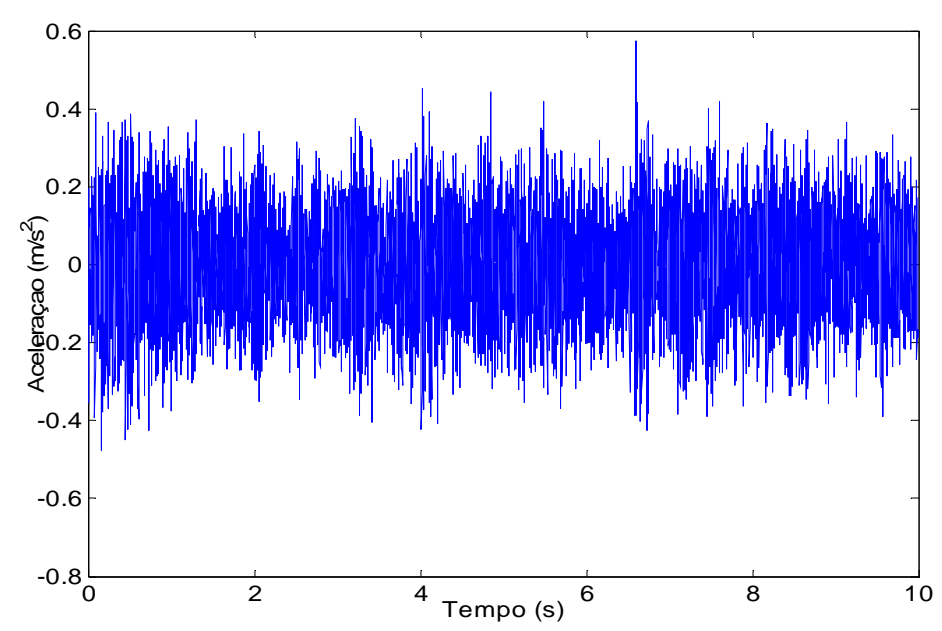

Figura 7.7: Sinal de aceleração - caso $A$ 
A partir dos sinais no domínio do tempo, coletados por meio dos ensaios experimentais para o caso $A$ e caso $B$, as estimativas das freqüências obtidas através do algoritmo de identificação com base no modelo ARMAV (Figura 6.1), para valores de $p$ variando no intervalo [2 20], podem ser vistas na Figura 7.8, nos diagramas de estabilidade.

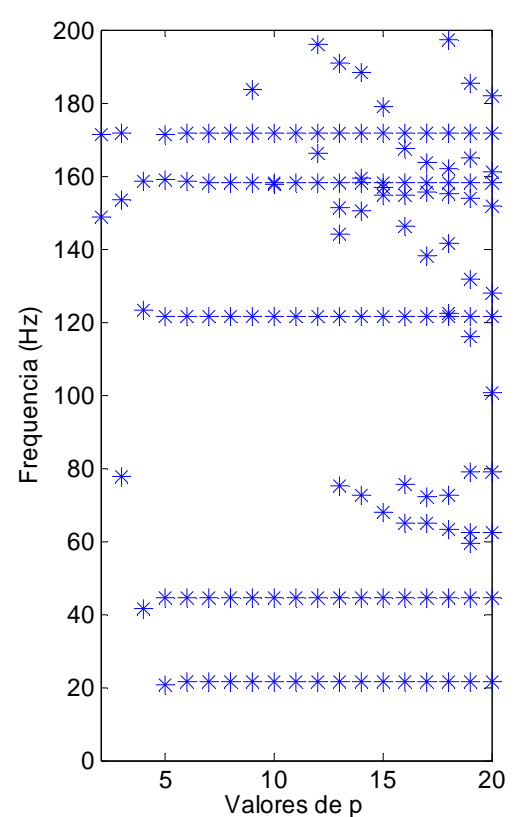

(a)

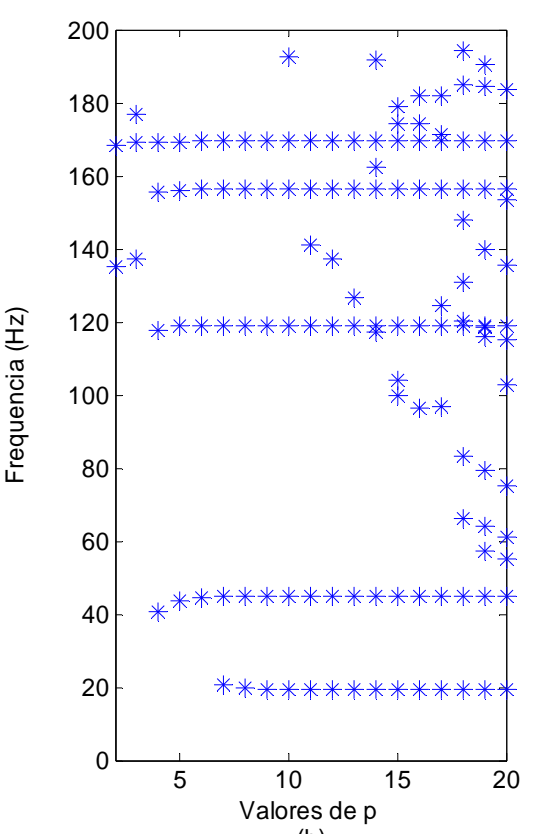

(b)

Figura 7.8: Diagramas de estabilidade - modelo ARMAV- (a) caso A; (b) caso B

Através da análise gráfica dos diagramas, pode-se notar que em ambos os casos, cinco freqüências se estabilizam no intervalo de $p$ pré-determinado, o que corresponde as cinco primeiras freqüências naturais do sistema, isto é, da placa de alumínio.

Para identificar as freqüências naturais do sistema na banda de interesse é necessário, porém, estimar a ordem do modelo ARMAV. Para tanto foi utilizado o critério BIC, apresentado no capítulo 5. Em ambos os casos, segundo o critério BIC, a ordem ideal que corresponde ao ponto de mínimo da curva é igual a $p=9$. A Figura 7.9 ilustra o gráfico dos valores de BIC em função dos valores de $p$, obtidos a partir da identificação correspondente ao caso $A$. 
A eliminação dos modos computacionais foi realizada através dos mesmos critérios de seleção natural mencionados no Capítulo 6, os quais foram utilizados nas identificações de sistemas a partir de dados simulados.

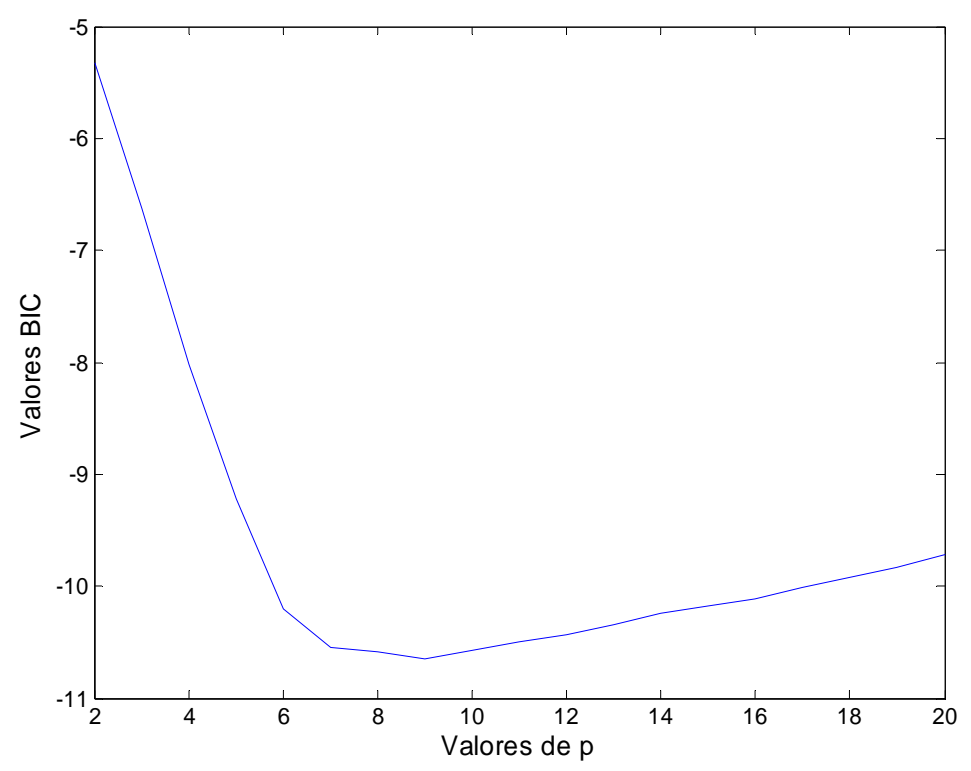

Figura 7.9: Critério BIC aplicado ao modelo ARMAV - caso A

Os resultados da identificação utilizando o modelo $\operatorname{ARMAV}(9,9)$ para ambos os casos $A$ e $B$ tais como as freqüências naturais e os fatores de amortecimento, podem ser vistos através da Tabela 7.2.

Tabela 7.2: Parâmetros modais identificados - modelo ARMAV-caso A e caso B

\begin{tabular}{|c|c|c|c||}
\hline \multicolumn{2}{|c|}{$\begin{array}{c}\text { ARMAV }(9,9) \\
\text { caso } A\end{array}$} & \multicolumn{2}{c|}{$\begin{array}{c}\text { ARMAV(9,9) } \\
\text { caso } B\end{array}$} \\
\hline \hline$\omega_{j}(\mathrm{~Hz})$ & $\zeta_{j}$ & $\omega_{j}(\mathrm{~Hz})$ & $\zeta_{j}$ \\
\hline 21.49 & 0.0474 & 19.48 & 0.0416 \\
\hline \hline 44.65 & 0.0008 & 44.99 & 0.0050 \\
\hline \hline 121.5 & 0.0033 & 118.9 & 0.0025 \\
\hline 158.2 & 0.0020 & 156.3 & 0.0009 \\
\hline \hline 171.6 & 0.0016 & 169.3 & 0.0007 \\
\hline
\end{tabular}

Para fins comparativos, a Tabela 7.3 traz as estimativas das freqüências naturais obtidas pelo modelo experimental, através dos picos do espectro de potência médio (EPM) (Figura 7.5), que representam as freqüências naturais do sistema para o caso $A$ e caso $B$, e pelo modelo de elementos finitos (MEF) (TSUNAKI, 1999). 
Tabela 7.3: Estimativas das freqüências naturais obtidas por diferentes modelos

\begin{tabular}{|c|c|c||}
\hline \multicolumn{3}{|c|}{ Freqüências $-\omega_{j}(\mathrm{~Hz})$} \\
\hline EPM-caso $A$ & EPM-caso $B$ & $M E F$ \\
\hline 21.12 & 19.21 & 20.00 \\
\hline \hline 44.63 & 44.63 & 48.40 \\
\hline 121.5 & 118.9 & 122.1 \\
\hline 158.0 & 156.4 & 156.6 \\
\hline \hline 171.6 & 169.4 & 177.0 \\
\hline
\end{tabular}

Através de uma análise comparativa, entre as freqüências obtidas na identificação a partir do modelo ARMAV e os valores apresentados na tabela acima obtidos pelo $M E F$ e EPM para o caso A, e em seguida ao caso B, os erros relativos percentuais máximos em relação a cada freqüência são calculados e apresentados na Tabela 7.4.

Tabela 7.4: Máximo erro relativo percentual - freqüências (ARMAV)

\begin{tabular}{|c|c|}
\hline \multicolumn{2}{|c|}{$\operatorname{Erro}(\%)-\omega_{j}$} \\
\hline caso $A$ & caso $B$ \\
\hline 6.933 & 2.669 \\
\hline 8.398 & 7.579 \\
\hline \hline 0.4938 & 2.691 \\
\hline \hline 1.011 & 0.1919 \\
\hline 3.146 & 4.548 \\
\hline
\end{tabular}

Analisando os valores da Tabela 7.4 pode-se notar que o erro em relação a freqüência não ultrapassou $8.5 \%$ no caso $A$ e permaneceu inferior a $8 \%$ no caso $B$.

Apesar da consistência dos resultados obtidos na identificação a partir do modelo ARMAV $(9,9)$ para ambos os casos $A$ e $B$, é conveniente que sejam realizadas as validações dos modelos. No entanto, a título de ilustração, será apresentado na Figura 7.10, somente o resultado do teste de validação do modelo $\operatorname{ARMAV}(9,9)$ para o caso $A$, porém, a mesma conclusão é válida para o modelo $\operatorname{ARMAV}(9,9)$ para o caso $B$. 


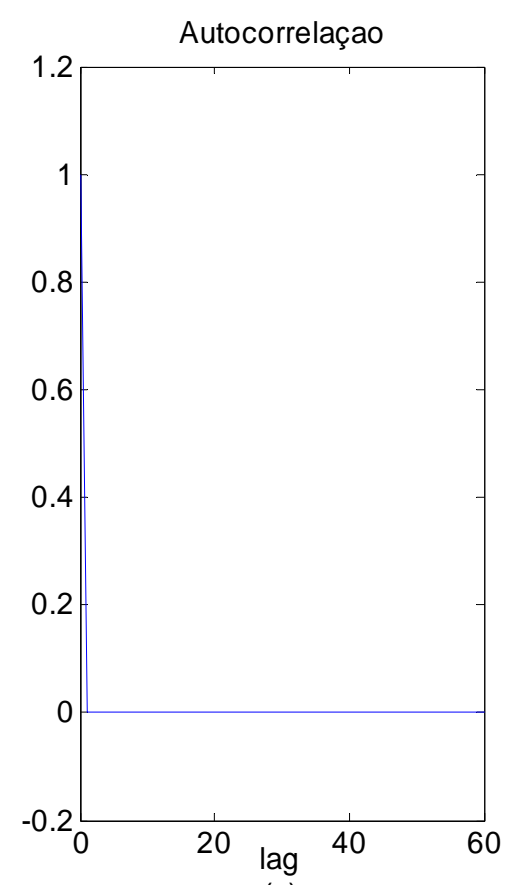

(a)

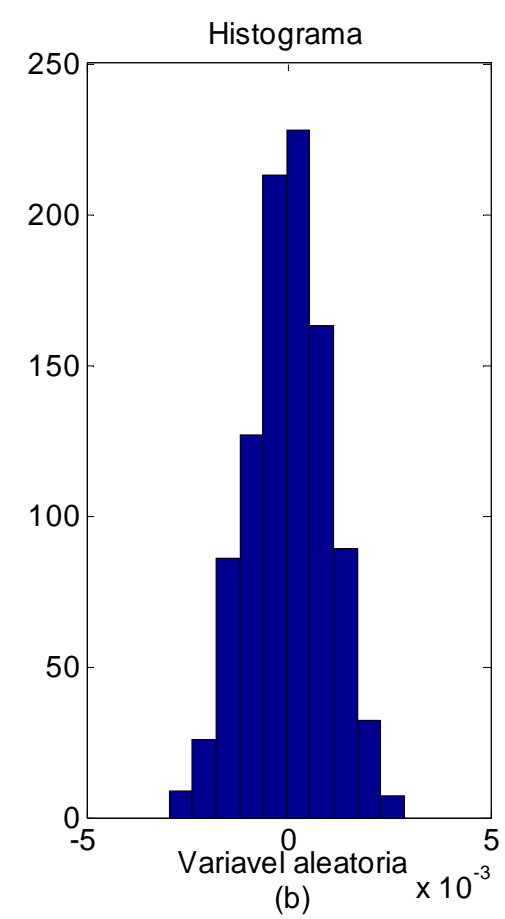

(b)

Figura 7.10: Teste do sinal residual da saída 2 (a) função de auto-correlação; (b) histograma (caso A)

Como pode ser observado através da Figura 7.10 (a), a função de autocorrelação do sinal residual correspondente a saída 2 , garante que o sinal seja próximo de um ruído branco. Já o histograma, Figura 7.10(b), indica que o sinal residual possui uma distribuição próxima da Gaussiana. O mesmo pode ser concluído com relação as saídas 1,3 e 4 .

Assim, pode-se concluir que os modelos ARMAV $(9,9)$ para ambos os casos $A$ e $B$, representam um bom ajuste dos dados pelos quais eles foram estimados.

Na identificação realizada através do algoritmo baseado no modelo ARV (Figura 6.2) foram utilizados somente os dados de resposta no domínio do tempo, ou seja, as quatro medidas de aceleração obtidas experimentalmente.

As Figuras 7.11 (a) e (b) trazem as estimativas das freqüências obtidas para o caso $A$ e caso $B$, respectivamente. Através dos diagramas, pode ser observada a estabilidade das cinco primeiras freqüências naturais da placa de alumínio no intervalo de variação de $p$ [2 20].

Com base na estabilidade das freqüências naturais do sistema, o algoritmo apresentado na Figura 6.3 é utilizado para realizar a seleção da estimativa que melhor represente os cinco primeiros modos da placa de alumínio. 


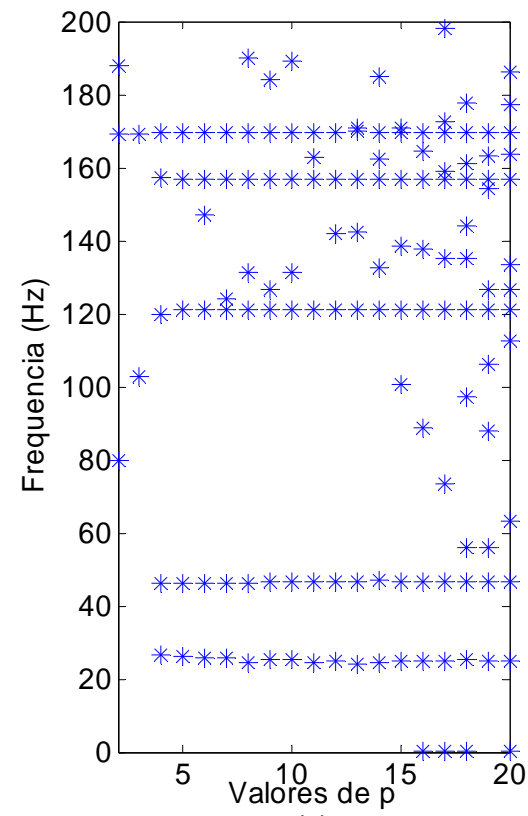

(a)

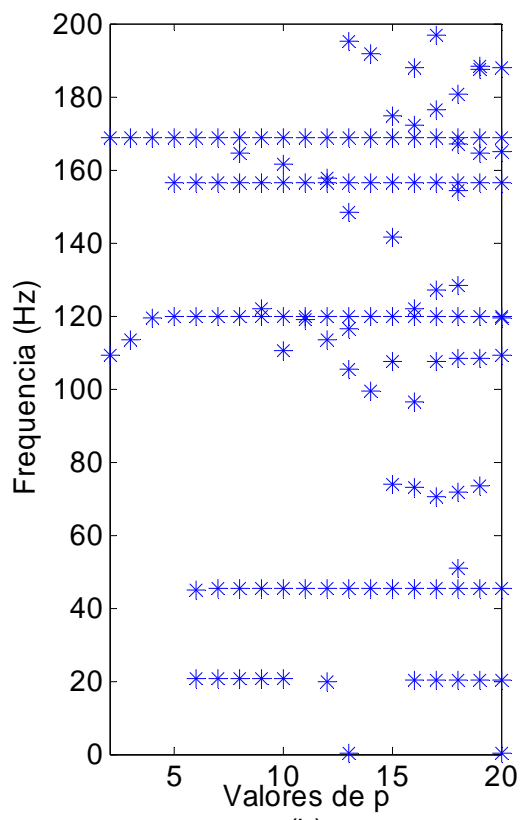

(b)

Figura 7.11: Diagramas de estabilidade - modelo ARV- (a) caso A; (b) caso B

Os valores de $\varepsilon, \varepsilon_{1}$ e $\varepsilon_{2}$, necessários no processo de escolha da melhor estimativa (Figura 6.3), foram considerados iguais àqueles utilizados nos casos simulados. Os parâmetros modais, que correspondem às cinco primeiras freqüências naturais e fatores de amortecimento da placa de alumínio, obtidos a partir dessa seleção, com as respectivas ordens $p_{j}$ do modelo ARV pelos quais foram determinados, estão dispostos na Tabela 7.5.

Tabela 7.5: Parâmetros Modais Identificados- Modelo ARV- (caso A e caso B)

\begin{tabular}{|c|c|c|c|c|c|}
\hline \multicolumn{3}{|c|}{$\begin{array}{c}\text { ARV }\left(p_{j}\right) \\
\text { caso } A\end{array}$} & \multicolumn{3}{c|}{$\begin{array}{c}\text { ARV }\left(p_{j}\right) \\
\text { caso B }\end{array}$} \\
\hline \hline$p_{j}$ & $\omega_{j}(\mathrm{~Hz})$ & $\zeta_{j}$ & $p_{j}$ & $\omega_{j}(\mathrm{~Hz})$ & $\zeta_{j}$ \\
\hline \hline 14 & 24.55 & 0.0536 & 18 & 20.04 & 0.0067 \\
\hline \hline 15 & 46.58 & 0.0466 & 13 & 45.42 & 0.0062 \\
\hline \hline 12 & 121.1 & 0.0029 & 13 & 119.6 & 0.0078 \\
\hline \hline 13 & 156.8 & 0.0054 & 12 & 156.2 & 0.0014 \\
\hline \hline 12 & 169.7 & 0.0026 & 11 & 168.7 & 0.0042 \\
\hline
\end{tabular}

Uma análise comparativa, análoga àquela realizada entre os resultados obtidos através da identificação a partir do modelo ARMAV, o $E P M$ e o $M E F$, será feita entre 
as freqüências naturais obtidas através do modelo ARV, o $M E F$ e o auto-espectro de potência médio da saída $(A E P M)$, para ambos os casos $A$ e $B$. Os gráficos dos dois autoespectros de potência médio obtido a partir das 4 saídas, de acordo com o ponto de excitação $A$ e $B$ são ilustrados na Figura 7.12.

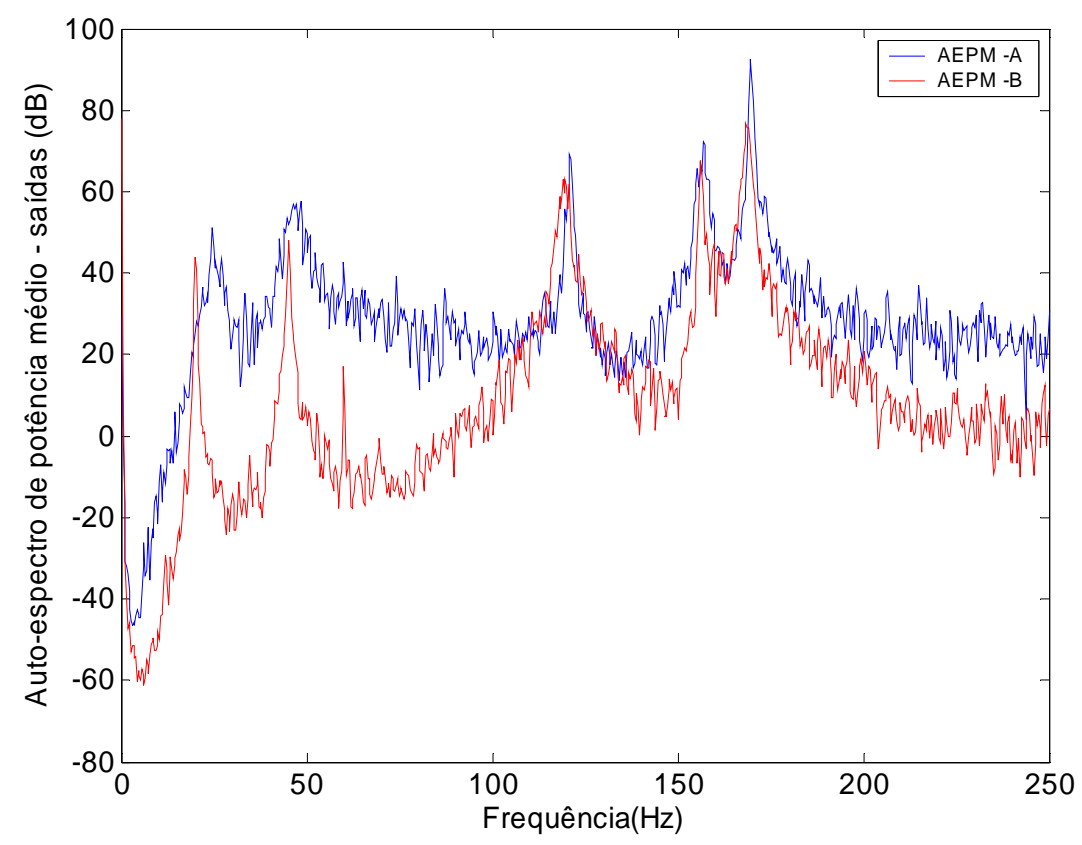

Figura 7.12: Auto-espectro de potência média de saída - pontos de excitação $\boldsymbol{A}$ e $\boldsymbol{B}$

As estimativas das freqüências naturais da placa para o caso $A$ e o caso $B$, obtidas experimentalmente através dos picos do gráfico do $A E P M$, podem ser vistas na Tabela 7.6.

Tabela 7.6: Estimativas das freqüências naturais obtidas pelos $A E P M$

\begin{tabular}{|c|c|}
\hline \multicolumn{2}{|c|}{ Freqüencias $-\omega_{j}(\mathrm{~Hz})$} \\
\hline AEPM-caso $A$ & AEPM-caso B \\
\hline 24.71 & 19.99 \\
\hline 46.44 & 45.18 \\
\hline 121.3 & 119.8 \\
\hline 156.9 & 156.3 \\
\hline 169.5 & 168.6 \\
\hline
\end{tabular}

Os erros relativos percentuais máximos em relação às freqüências para o caso $A$ e caso $B$, quando comparados as freqüências naturais obtidas a partir do modelo ARV, $A E P M$ e o $M E F$ são apresentados na Tabela 7.7. 
Tabela 7.7: Máximo erro relativo percentual - freqüências (ARV)

\begin{tabular}{|c|c|}
\hline \multicolumn{2}{|c|}{$\operatorname{Erro}(\%)$} \\
\hline \hline$\omega_{j}-$ caso $A$ & $\omega_{j}-$ caso $B$ \\
\hline \hline 18.53 & 0.1996 \\
\hline 3.907 & 6.561 \\
\hline \hline 0.8258 & 2.090 \\
\hline 0.1276 & 0.2561 \\
\hline \hline 4.3017 & 4.920 \\
\hline
\end{tabular}

De acordo com os resultados obtidos na Tabela 7.7, pode-se observar que o erro em relação à freqüência, para o caso $B$ permaneceu inferior a $7 \%$ enquanto que para $o$ caso $A$ alcançou quase $19 \%$.

Comparando os resultados da identificação obtidos através do modelo ARMAV (Tabela 7.2) e modelo ARV (Tabela 7.5), pode-se notar a ocorrência de uma expressiva variação na primeira freqüência natural, do caso $A$. Provavelmente, o aumento na primeira freqüência identificada através do modelo ARV, deve ter sido ocasionada devido à influência da massa da armadura do excitador (shaker). Essa influência pode diminuir de acordo com a localização do excitador na estrutura em teste, quando se utiliza no procedimento de identificação apenas as respostas do sistema. Quanto mais próximo da base de fixação o excitador estiver posicionado, menor é a sua influência, conforme pode ser notado no caso $B$.

É importante notar também que a influência do excitador torna-se menor nas freqüências mais altas.

É importante observar que os picos dos auto-espectros de potência médio das saídas, correspondentes as 2 primeiras freqüências (Figura 7.12), estão deslocados à direita, comparados com os picos dos espectros de potência médio das FRFs (Figura 7.5), o que condiz com o resultado obtido na identificação através do modelo ARV e ARMAV, respectivamente.

Para que os modos de vibrar, identificados na placa de alumínio, possam ser representados graficamente, é necessário que se tenha um número suficiente de saídas para obter uma boa resolução espacial. Para tanto, como não havia disponibilidade de equipamentos para coletar um número grande de saídas simultaneamente, utilizou-se a 
seguinte estratégia. Conforme mostra a Figura 7.13, foram marcados 81 pontos de saída na placa, distribuídos de maneira uniforme. Em seguida, foram realizados 27 ensaios, sendo que o único ponto de excitação foi mantido na mesma posição da placa, segundo mostra a Figura 7.13, para todos os ensaios. Dentre os quatro acelerômetros utilizados, um deles foi tomado como referência e mantido na posição $(2,2)$ da placa em todos os ensaios. Os outros 3 acelerômetros foram deslocados de ensaio para ensaio, da direita para a esquerda, percorrendo assim os 81 pontos distribuídos sobre a placa.

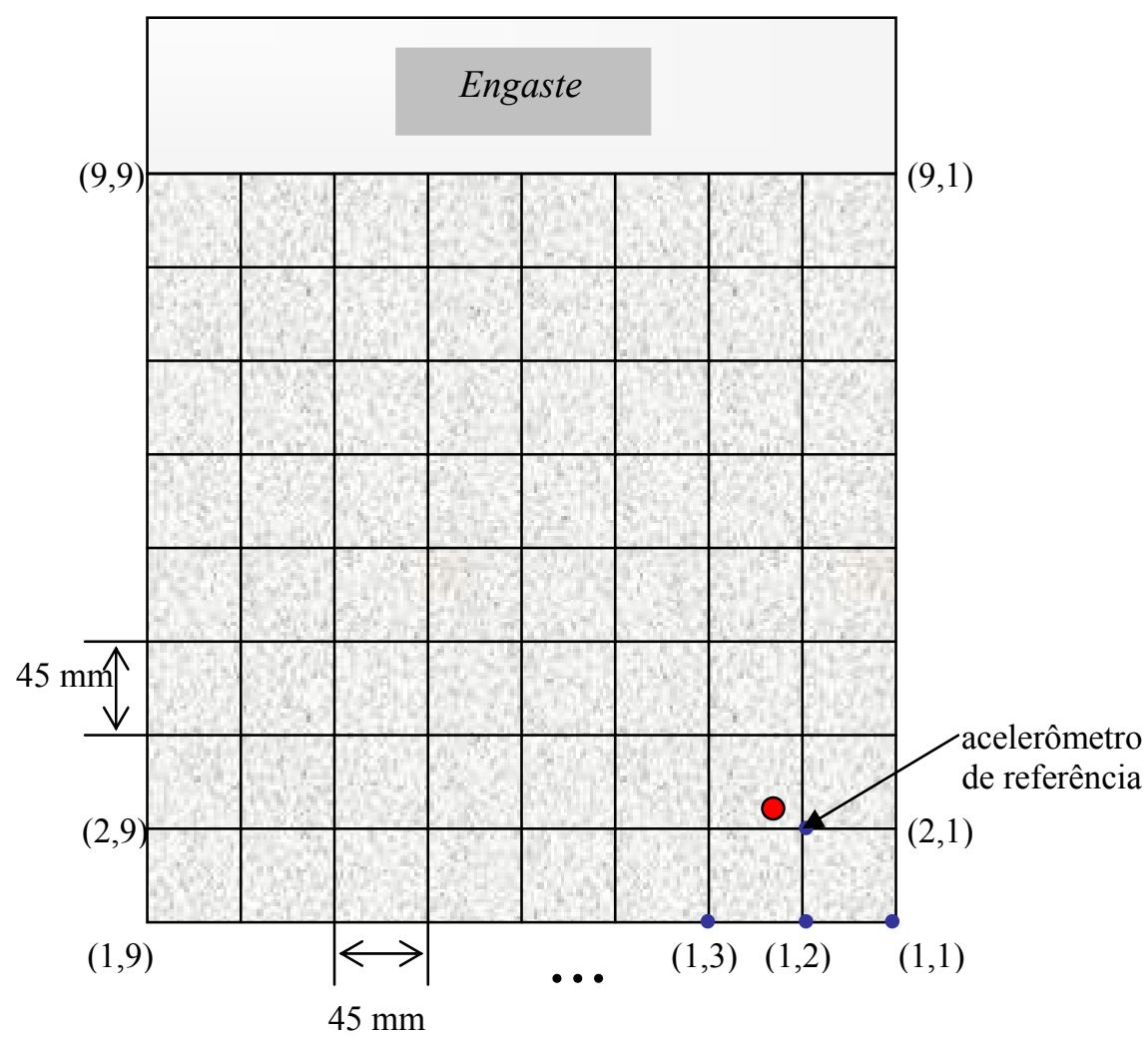

Figura 7.13: Distribuição dos pontos de excitação e de resposta sobre a placa utilizada na representação gráfica dos modos

Para cada ensaio, foram obtidas cinco freqüências naturais pertencentes a banda de interesse, com seus respectivos fatores de amortecimento e modos de vibrar com quatro coordenadas complexas, sendo uma delas a referência adotada.

A Tabela 7.8 mostra a média das freqüências identificadas nos 27 ensaios (Médfreq), com seus respectivos desvios-padrão (DP freq). 
Tabela 7.8: Médias e desvios-padrão das freqüências

\begin{tabular}{|c||c||}
\hline Méd_freq & DP_freq \\
\hline 20.43 & 0.6924 \\
\hline 47.93 & 2.580 \\
\hline 121.7 & 2.043 \\
\hline \hline 156.3 & 2.751 \\
\hline \hline 171.0 & 2.386 \\
\hline
\end{tabular}

Para cada um dos 27 ensaios, os modos complexos foram normalizados de maneira que a norma da coordenada de referência fosse igual a 1. Em seguida, as 81 coordenadas complexas de cada modo, após serem convertidas para valores reais, segundo o critério apresentado na seção 6.3.1 do capítulo 6 , foram utilizadas para representar graficamente os cinco primeiros modos de vibrar da placa de alumínio, conforme exibidos nas Figuras 7.14 a 7.18.

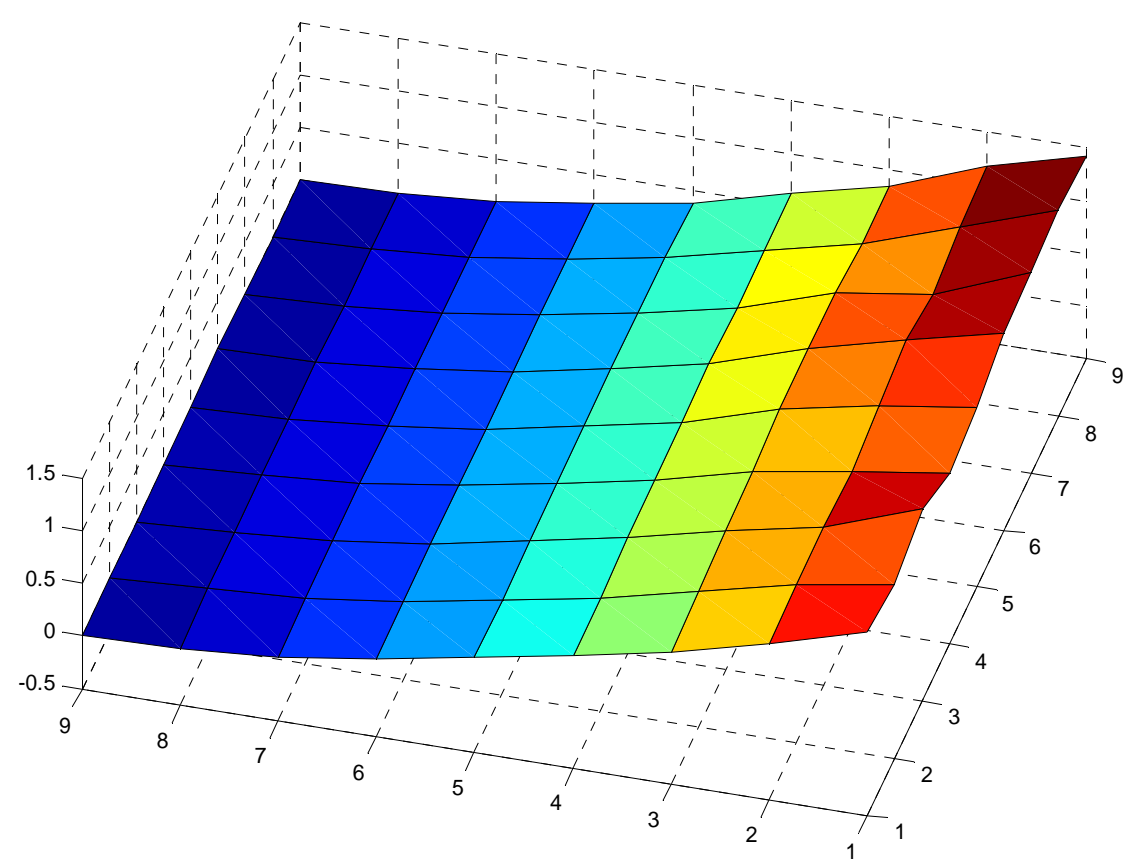

Figura $7.14: 1^{\circ}$ modo de vibrar da placa da alumínio 


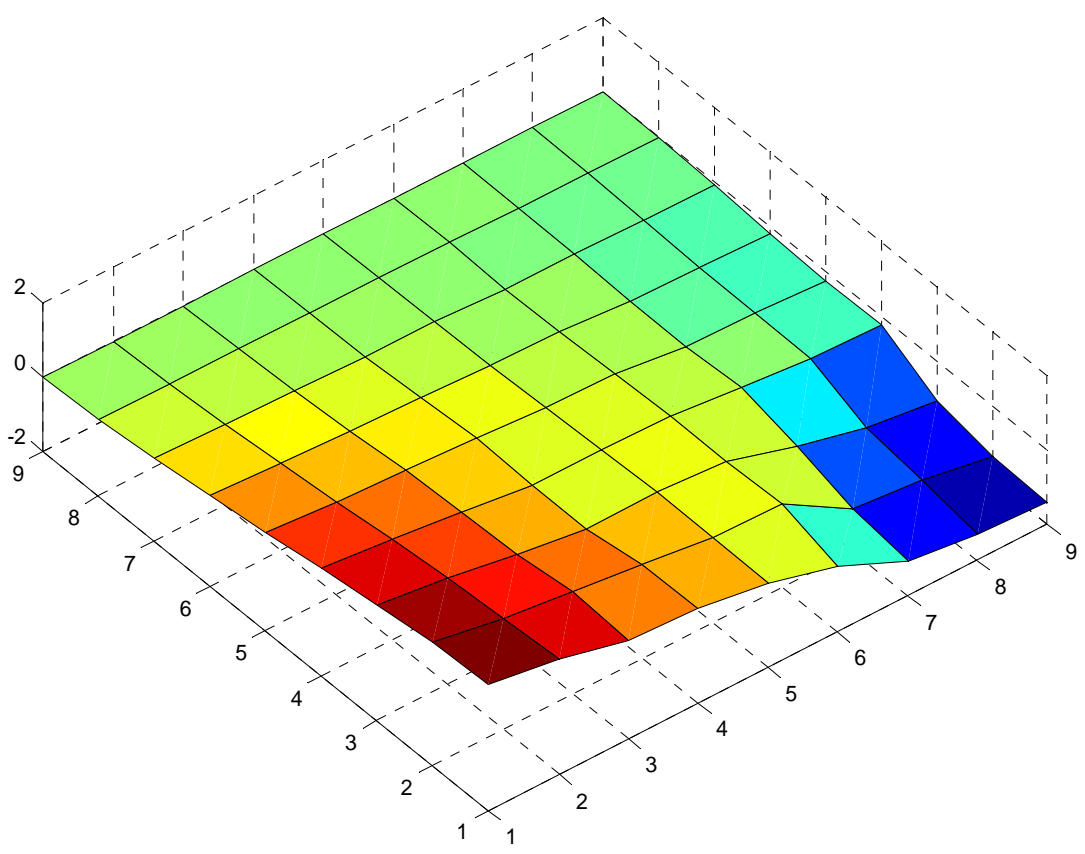

Figura $7.15: 2^{\circ}$ modo de vibrar da placa da alumínio

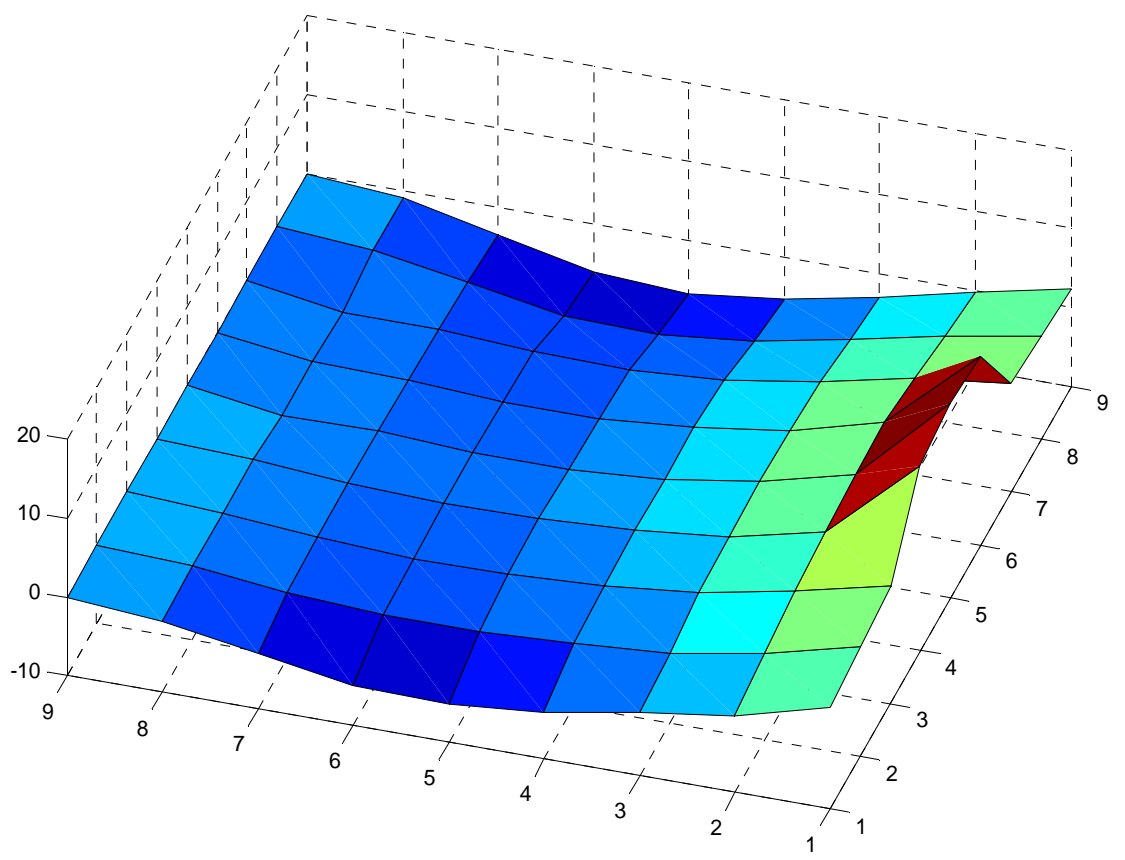

Figura $7.16: 3^{\circ}$ modo de vibrar da placa da alumínio 


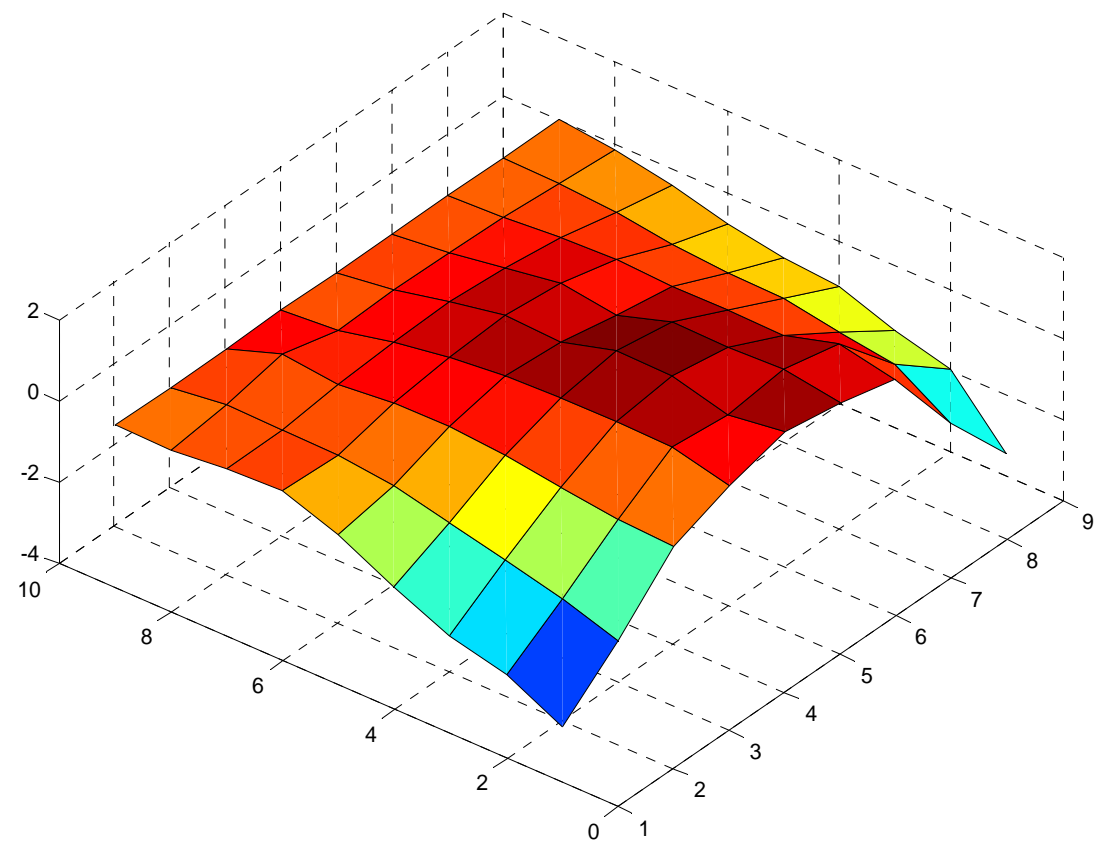

Figura $7.17: 4^{\circ}$ modo de vibrar da placa da alumínio

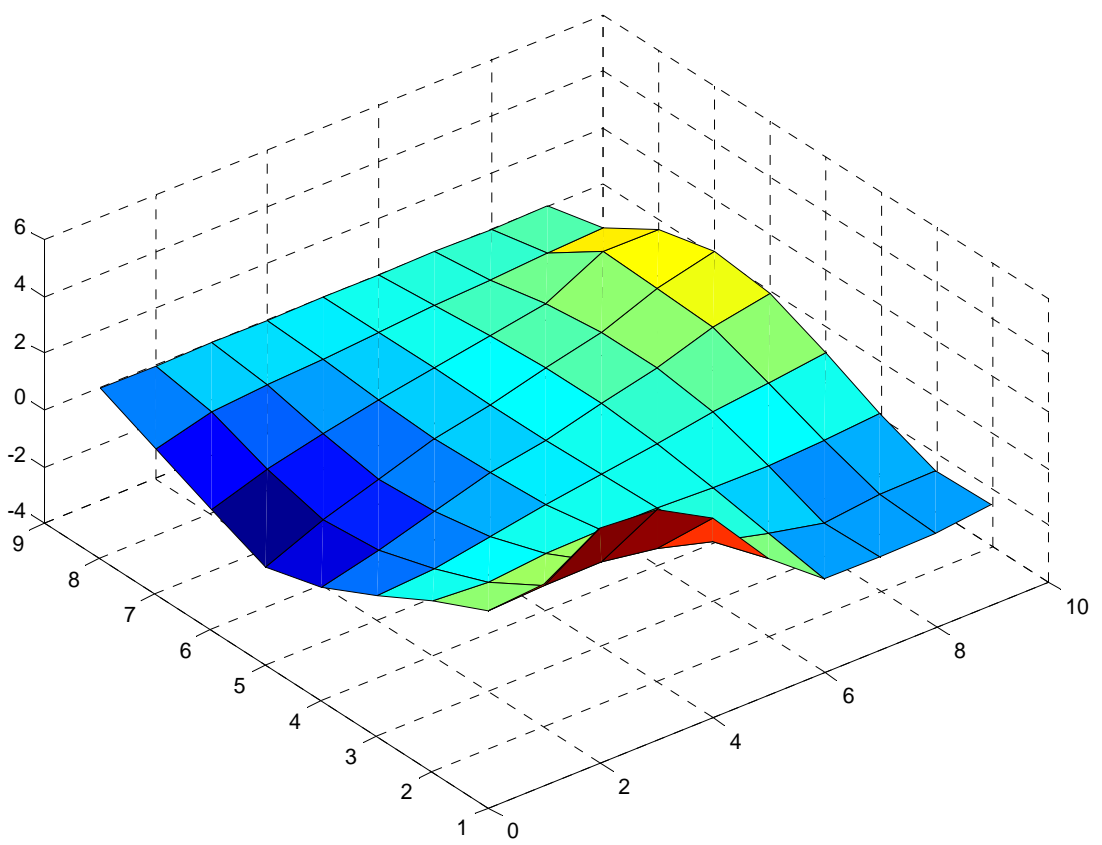

Figura $7.18: 5^{\circ}$ modo de vibrar da placa da alumínio 
A representação gráfica dos modos foi obtida através do algoritmo de identificação com base no modelo ARMAV. É importante observar que só foi possível desenhar a forma dos modos, ainda que de maneira razoável, pelo fato da estrutura em estudo, no caso a placa de alumínio, ser fracamente não proporcional. Isso se deve ao fato de que a aproximação dos modos complexos no caso de amortecimento não proporcional para modos reais é considerada aceitável, somente se, a estrutura possuir um baixo fator de amortecimento. Caso a estrutura em estudo tivesse um amortecimento fortemente não proporcional, a representação gráfica dos modos na forma convencional não seria possível. 


\section{Conclusões e Propostas Futuras}

\subsection{CONCLUSÕES GERAIS}

Este trabalho desenvolveu um estudo sobre técnicas de identificação no domínio do tempo aplicada à análise modal de estruturas mecânicas, através dos modelos ARMAV e ARV.

Para verificar a eficiência dos algoritmos de identificação, foi utilizada a aproximação dos mínimos quadrados para encontrar os coeficientes do modelo ARMAV, e a dos mínimos quadrados modificada na estimação dos coeficientes do modelo ARV. As técnicas de identificação foram aplicadas a dados provenientes de simulações numéricas e de um ensaio experimental.

Primeiramente, dois modelos de sistemas mecânicos distintos com características interessantes do ponto de vista do problema de identificação foram considerados. Com esses modelos, buscou-se analisar situações próximas do real a partir de dados de saída contaminados por ruído e de um número reduzido de medidas de respostas.

Posteriormente, uma placa de alumínio engastada em um dos lados foi submetida à análise modal experimental para que os dados obtidos no domínio do 
tempo pudessem ser utilizados como dados de entrada para os algoritmos de identificação.

Em geral, a partir dos resultados obtidos, pode-se verificar a eficiência das técnicas de identificação para estimar os parâmetros modais, dentro da análise considerada, através de ambos os modelos ARMAV e ARV.

Quanto à determinação da ordem do modelo ARMAV, pode-se observar que em algumas situações, o critério BIC não foi eficaz, ora sobre-determinando ora subestimando o modelo, chegando assim a prejudicar a identificação dos parâmetros modais em certos casos.

Já a técnica proposta, que contribuiu para estimar a ordem que melhor representa o sistema físico na faixa de freqüência de interesse, a partir da identificação baseada no modelo ARV, mostrou-se eficiente em todas as situações analisadas. Esta técnica, que é baseada no princípio de que os modos estruturais tendem a se estabilizar para diferentes estimativas, colaborou também para automatizar o procedimento de estimação dos parâmetros modais, exigindo pouca intervenção do analista.

Em relação à aplicação experimental, pode-se observar que os valores das freqüências naturais obtidos na identificação do modelo experimental através do modelo ARMAV e ARV revelaram-se ajustados com os resultados obtidos a partir do espectro de potência médio das FRFs e dos auto-espectros de saída, obtidos experimentalmente. Além disso, os resultados mostraram-se coerentes com aqueles obtidos através do modelo de elementos finitos na mesma estrutura (TSUNAKI, 1999).

Porém, a partir de uma análise comparativa dos resultados obtidos através do modelo ARMAV e ARV, pode-se notar uma variação significativa principalmente da freqüência correspondente ao primeiro modo da placa de alumínio. O aumento na primeira freqüência, identificada a partir do modelo ARV, provavelmente ocorreu devido o efeito da massa do excitador. Como visto, esse efeito pode variar de acordo com a localização do excitador na estrutura, além de exercer uma maior influência nas freqüências mais baixas.

Quanto às formas dos cinco primeiros modos da placa de alumínio identificados através do modelo ARMAV, mostraram-se boa semelhança com aquelas obtidas nos trabalhos de (TSUNAKI,1999) e (PAZIANI, 2002).

Por fim, com base nas estimativas obtidas a partir dos dados provenientes de simulações numéricas e do ensaio experimental, pode-se concluir que o algoritmo de identificação utilizando o modelo ARMAV, e o modelo ARV aliado a técnica proposta, 
desempenharam suas funções de forma eficiente para os modelos considerados neste trabalho, na identificação das freqüências naturais, fatores de amortecimento e modos de vibrar.

\subsection{PROPOSTA PARA FUTUROS DESENVOLVIMENTOS}

Algumas sugestões para desenvolvimentos futuros, que surgiram como conseqüência do estudo realizado neste trabalho, são apresentadas a seguir:

- Realizar um ensaio experimental excitando a estrutura através da base, com o intuito de diminuir ou até mesmo excluir a interferência do excitador, na identificação dos parâmetros modais da estrutura a partir do modelo ARV;

- Desenvolver técnica utilizando o critério de confiança modal (MAC), e acoplá-la à técnica proposta neste trabalho, baseada no diagrama de estabilidade das freqüências, para que seja possível realizar a identificação modal de forma satisfatória, caso haja acoplamento dos modos, como no caso da ocorrência do fenômeno de flutter (REBOLHO, 2006).

- Estudar e verificar o efeito de uma excitação aleatória, diferente do ruído branco, na análise modal de estruturas mecânicas, utilizando os algoritmos de identificação com base nos modelos ARMAV e ARV.

- Investigar a aplicabilidade dos algoritmos de identificação a partir dos modelos ARMAV e ARV para o caso onde a excitação do sistema não assume uma distribuição gaussiana e é considerada não-estacionária, por meio de um modelo simulado. 


\section{Referências Bibliográficas}

AGUIRRE, L. A.; RODRIGUES, G.G.; JÁCOME, C. R. F. (1998). Identificação de Sistemas não lineares utilizando modelos NARMAX polinomiais - Uma revisão e novos resultados. SBA Controle \& Automação, v.9, n.2, p.90-106.

AKAIKE, H. (1969). Fitting autoregressive models for prediction. Annals of the Institute of Statistical Mathematics, Tokyo, v.1, p.243-347.

. (1974). A new look at the statistical model identification. IEEE Transactions on Automatic Control, New York, v.AC-19, n.6, p.716-723, Dec.

ALLEMANG, R. J.; BROWN, D. L. (1982). A correlation coefficient for modal vector analysis. In: INTERNATIONAL MODAL ANALYSIS CONFERENCE, 1., 1982, Orlando. Proceedings... Bethel: Society for Experimental Mechanics, p.110-116.

ANDERSEN, P. (1997). Identification of civil engineering structures using ARMA models. 258p. PhD Thesis - Departament of Building Technology and Structural Engineering, Aalborg University, Denmark, 1997.

BAZÁN, F.S.V. (1993). Desenvolvimento de ferramentas de identificação paramétrica de sistemas mecânicos no domínio do tempo. 90p. Tese (Doutorado) - Faculdade de Engenharia Mecânica, Universidade Estadual de Campinas, Campinas, 1993.

BENDAT, J.S.; PIERSOL, A.G. (1993). Engineering applications of correlation and spectral analysis. $2^{\text {nd }}$ ed. New York: John Wiley.

BODEUX, J.B.; GOLINVAL, J.C. (2001). Application of ARMAV models to the identification and damage detection of mechanical and civil engineering structures. Smart Materials and Structures, Bristol, v.10, n.3, p.479-489, June. 
. (2003). Modal identification and damage detection using the data-driven stochastic subspace and ARMAV methods. Mechanical Systems and Signal Processing, London, v.17, n.1, p.83-89, Oct.

BOX, G.P.; JENKINS, G.M. (1976). Time series analysis forecasting and control. Englewood Cliffs: Prentice Hall.

BRAUN, S. (1986). Mechanical signature analysis: theory and applications. London: Academic Press.

BROWN, D.L. et al. (1979). Parameter estimation techniques for modal analysis. Warrendale: SAE. (SAE Technical Paper Series, 790221).

BROWN, R.G.; HWANG, P.Y. (1992). Introduction to random signals and applied kalman filtering. New York: John. Wiley.

CARDOSO, A. (2006). A transformada wavelet aplicada à identificação modal modal operacional. 147 p. Tese (Doutorado) - Escola de Engenharia de São Carlos, Universidade de São Paulo, São Carlos, 2006.

CHEN, C.T. (1999). Linear system theory and design. New York: Oxford University Press.

COOLEY, J.W.; TUKEY, J.W. (1965). An algorithm for the machine calculation of complex fourier series. Mathematics of Computation, Menasha, v.19, n.90, p.297301, Apr.

D'ALESSANDRO, G. et al. (1991). ARMAV model technique for multiple input/output system identification. In: INTERNATIONAL MODAL ANALYSIS CONFERENCE, 11.,, 1993, Kissimmee. Proceedings... Bellingham: SPIE. v.1, p.246-249. (SPIE, 1923) 
DAVIES, P.; HAMMOND, J.K. (1984). A comparison of fourier and parametric methods for structural system identification. Journal of Vibration and Acoustics, New York, v.106, p.40-48, Jan.

DEBLAUWE, F.; BROWN, D.L.; ALLEMANG, R.J. (1987). The polyreference time domain technique. In: INTERNATIONAL MODAL ANALYSIS CONFERENCE, 5. 1987, London. Proceedings... Schenectady: Union College. p.832-845.

DESFORGES, M.J.; COOPER, J.E.; WRIGHT, J.R. (1995). Spectral and modal parameter estimation from output-only measurements. Mechanical Systems and Signal Processing, London, v.9, n.2, p.169-186, June.

DUONG, H.N.; LANDAU, D.I. (1996). An IV based criterion for model order selection. Automatica, New York, v.32, n.6, p.909-914, Dec.

EWINS, D.J. (1984). Modal testing: theory and practice. Letchworth: Research Studies Press.

FASSOIS, S.D. (2001). MIMO LMS-ARMAX identification of vibrating structurespart I: the method. Mechanical Systems and Signal Processing, London, v.15, n.4, p.723-735, Dec.

FASSOIS, S.D.; LEE, J.D. (1993). On the problem of stochastic experimental modal analysis based on multiple-excitation multiple-response data, part II: the modal analysis approach. Journal of Sound and Vibration, London, v.161, n.1, p.57-87, Sept.

GARIBALDI, L.; GIORCELLI, E.; PIOMBO, B.A.D. (1998). ARMAV techniques for traffic excited bridges. Journal of Vibration and Acoustics, New York, v.120, n.3, p.713-718, July.

GARIBALDI, L. et al. (2001). CVA and ARMAV: performance comparison over real data. Journal of Intelligent Material Systems and Structures, London, v.12, p.577588, Aug. 
GAUTIER, P.E.; GONTIER, C.; SMAIL, M. (1995). Robustness of an ARMA identification method for modal analysis of mechanical systems in the presence of noise. Journal of Sound and Vibration, London, v.179, n.2, p.227-242, Sept.

GERSCH, W. (1974). On the achievable accuracy of structural system parameter estimates. Journal of Sound and Vibration, London, v.34, n.1, p.63-79, Nov.

GERSCH, W.; FOUTCH, D.A. (1974). Least squares estimates of structural system parameters using covariance function data. IEEE Transactions on Automatic Control, New Yoprk, v.AC-19, n.6, p.898-903, Dec.

GERSCH, W.; NIELSEN, N.N.; AKAIKE, H. (1973). Maximum likelihood estimation of structural parameters from random vibration data. Journal of Sound and Vibration, London, v.31, n.3, p.295-308, Apr.

GERSCH, W.; TAOKA, G.T.; LIU, R. (1976). Structural system parameter estimation by two-stage least-squares method. Journal of Engineering Mechanics Division, New York, v.102, n.EM5, p.883-899, Oct.

GHANEM, R.; SHINOZUKA, M. (1995). Structural-system identification I: theory. Journal of Engineering Mechanics, New York, v.121, n.2, p.255-264, Feb.

GIORCELLI, E. et al. (1994). Modal analysis and system identification using ARMAV models. In: INTERNATIONAL MODAL ANALYSIS CONFERENCE, 12., 1994, Honolulu. Proceedings... Bethel: Society for Experimental Mechanics. v.1, p.676-680.

. (1996). ARMAV analysis of queensborough bridge ambient data. In: INTERNATIONAL MODAL ANALYSIS CONFERENCE, 14., 1996, Dearborn. Proceedings... Bethel: Society for Experimental Mechanics. v.1, p.466-469.

GOHBERG, I.; LANCASTER, P; RODMAN, L. (1982). Matrix polynomials computer science and applied mathematics. New York: Academic Press. 
GONTIER, C.; SMAIL, M.; GAUTIER, P.E. (1993). A time domain method for the identification of dynamic parameters of structures. Mechanical Systems Signal Processing, London, v.7, n.1, p.45-56, Jan.

HERMANS, L. et al. (1997). Modal parameter extraction from in-operation data. In: INTERNATIONAL MODAL ANALYSIS CONFERENCE, 15., 1997, Orlando. Proceedings... Bethel: Society for Experimental Mechanics. v.1, p.531-539.

HUANG, C.S. (2001). Structural identification from ambient vibration measurement using the multivariate AR model. Journal of Sound and Vibration, London, v.241, n.3, p.337-359, July.

HUANG, C.S.; YEH, C.H. (1999). Some properties of randomdec signatures. Mechanical Systems and Signal Processing, London, v.13, n.3, p.491-507, July.

IBRAHIM, S.R.; MIKULCIK, E.C. (1973). A time domain modal vibration test technique. Shock and Vibration Bulletin, v.43, n.4, p.21-37.

. (1977). A method for the direct identification of vibration parameters from the free response. Shock and Vibration Bulletin, v.47, n.4, p.184-198.

JAMES III, G.H., CARNE, T.G. AND LAUFFER, J.P. (1995), The natural excitation technique (NExT) for modal parameter extraction from operating structures. Modal Analysis: The International Journal of Analytical and Experimental Modal Analysis, v. 10, n. 4, p.260-277, Oct.

JUANG, J.N. (1994). Applied system identification. Englewood Cliffs: PrenticeHall.

JUANG, J.N.; PAPPA, R.S. (1985). An eigensystem realization algorithm for modal parameter identification and model reduction. Journal of Guidance Control and Dynamics, Washington, v.8, n.5, p.620-627. 
KADAKAL, U.; YÜZÜGÜLLÜ, Ö. (1996). A comparative study on the identification methods for the autoregressive modelling from the ambient vibration records. Soil Dynamics Earthquake Engineering, Oxford, v.15, n.1, p.45-49, July.

KAY, S.M. (1988). Modern spectral estimation: theory and application. Englwood Cliffs: Prentice-Hall.

LARBI, N.; LARDIES, J. (2000). Experimental modal analysis of a structure excited by a random force. Mechanical Systems and Signal Processing, London, v.14, n.2, p.181-192, Sept.

LEE, A.C.; CHEN, J.H. (1989). Modal analysis for randomly excited structural system with unmeasured input. Journal of Sound and Vibration, London, v.132, n.1, p.101-113, July.

LIANG, G.; WILKES, D.M.; CADZOW, J.A. (1993). ARMA model order estimation based on the eigenvalues of the covariance matrix. IEEE Transactions on Signal Processing, New York, v.41, n.10, p.3003-3009, Sept.

LJUNG, L. (1987). System identification: theory for the user. Englewood Cliffs: Prentice Hall

LJUNG, L.; SÖDERSTROM, T. (1983). Theory and practice of recursive identification. Cambridge: MIT Press.

MAIA, N.M.M.; SILVA, J.M.M. (1997). Theoretical and experimental modal analysis. New York: John Wiley.

MARPLE JR., S.L. (1988). Digital spectral analysis with application. Englewood Cliffs: Prentice Hall.

MATHIAS, M.H. (1998). Aplicação da técnica paramétrica ARMA de processamento de sinais na caracterização de estruturas mecânicas. $117 \mathrm{p}$. Tese (Doutorado) - Faculdade de Engenharia Mecânica, Universidade Estadual de Campinas, Campinas, 1998. 
MICKLEBOROUGH, N.C.; PI, Y.L. (1989). Modal parameter identification using Ztransforms. International Journal for Numerical Methods in Engineering, London, v.28, p.2307-2321, Mar.

PAPAKOS, V.; FASSOIS, S.D. (2003). Multichannel identification of aircraft skeleton structures under unobservable excitation: a vector AR/ARMA framework. Mechanical Systems and Signal Processing, London, v.17, n.1, p.1-20, Oct.

PAZIANI, F.T. (2002). Identificação de parâmetros modais no domínio do tempo: método IDT. 107p. Dissertação (Mestrado) - Escola de Engenharia de São Carlos, Universidade de São Paulo, São Carlos, 2002.

PEETERS, B.; DE ROECK, G. (2001). Stochastic system identification for operational modal analysis: a review. Journal of Dynamic Systems, Measurement, and Control, New York, v.123, n.4, p.659-667, Dec.

PEETERS, B; DE ROECK, G.; ANDERSEN, P. (1999). Stochastic system identification: uncertainty of the estimated modal parameters. In: INTERNATIONAL MODAl ANAlysis CONFERENCE, 17., 1999, Kissimmee. Proceedings... Local: Editora. p.231-237.

PEETERS, B. et al. (1998). Comparison of system identification methods using operational data of a bridge test. In: INTERNATIONAL CONFERENCE ON NOISE AND VIBRAting EngIneERING, 23., 1998, Belgium. Proceedings... Belgium: ISMA. p.923-930.

PETSOUNIS, K.A.; FASSOIS, S.D. (2001). Parametric time-domain methods for the identification of vibrating structures - a critical comparison and assessment. Mechanical Systems and Signal Processing, London, v.15, n.6, p.1031-1060, Nov.

PI, Y.L.; MICKLEBOROUGH, N.C. (1989). Modal identification of vibration structures using ARMA model. Journal of Engineering Mechanics, New York, v.115, n.10, p.2232-2250, Oct. 
PIOMBO, B. et al. (1993). Structures identification using ARMAV models. In: INTERNATIONMAL MODAL ANALYSIS CONFERENCE, 11., 1993, Kissimmee. Proceedings... Bellingham: SPIE. v.1, p.588-592. (SPIE, 1923).

PUKKILA T.M.; KRISHNAIAH, P.R. (1988). On the use of autorregressive order determination criteria in multivariate white noise tests. IEEE Transactions on Acoustics, Speech, and Signal Processing, New York, v.36, n.9, p.1396-1403, Sept.

REBOLHO, D.C. (2006). Um método para identificação de parâmetros modais em tempo real. 129p. Tese (Doutorado) - Escola de Engenharia de São Carlos, Universidade de São Paulo, São Carlos, 2006.

RISSANEN, J. (1983). A universal prior for the integers and estimation by minimum description length. Annals of Statistics, v.11, n.2, p.416-431.

SCHWARZ, G. (1978). Estimation of the dimension of a model. Annals of Statistics, Hayward, v.6, n.2, p.461-464, Mar.

SCZIBOR, V. (2002). Identificação modal de uma estrutura aeronáutica via algoritmo de realização de sistemas. 104p. Dissertação (Mestrado) - Escola de Engenharia de São Carlos, Universidade de São Paulo, São Carlos, 2002.

SHINOZUKA, M.; GHANEM, R. (1995). Structural system identification II: experimental verification. Journal of Engineering Mechanics, New York, v.121, n.2, p.265-273, Feb.

SMAIL, M.; THOMAS, M. (1999). Assessment of optimal ARMA model orders for modal analysis. Mechanical Systems and Signal Processing, London, v.13, n.5, p.803-819, Feb.

SÖDERSTRÖM, T.; STOICA, P. (1989). System identification. Englewood Cliffs: Prentice Hall. 
TANEGUTI, L.Y. (1997). Análise de desempenho do método de identificação ITD. 178p. Tese (Doutorado) - Escola de Engenharia de São Carlos, Universidade de São Paulo, São Carlos, 1997.

TSUNAKI, R.H. (1999). Identificação automatizada de modelos dinâmicos no espaço de estado. 143p. Tese (Doutorado) - Escola de Engenharia de São Carlos, Universidade de São Paulo, São Carlos, 1999.

VAN DEN BOOM, A.J.W.; VAN DEN ENDEN, A.W.M. (1974). The determination of the orders of process and noise dynamics. Automatica, New York, v.10, p.245256, Nov.

VOLD, H. et al. (1982). A muti-inut modal estimation algorithm for minicomputers. Warrendale: SAE. (SAE Technical Papers Series, n.820194).

XIA HE; DE ROECK, G. (1997). System identification of mechanical structures by a high-order multivariate autoregressive model. Computers \& Structures, v.64, n.4, p.341-351.

WALD, A. (1947). Sequential analysis. New York: John Wiley.

WEI, W.W.S. (1990). Time series analysis: univariate and multivariate methods. Redwood City: Addison-Wesley.

YANG, Q.J. et al. (1994). A system theory approach to multi-input multi-output modal parameters identification methods. Mechanical System and Signal Processing, London, v.8, n.2, p.159-174, Nov.

YOUNG, P. (1976). Some observations on instrumental variable methods of timeseries analysis. International Journal of Control, London. v.23, n.5, p.593-612.

YOUNG, P.; JAKEMAN, A. (1979). Refined instrumental variable methods of recursive time-series analysis - part I - single input, single output systems. International Journal Control, London, v.29, n.1, p.1-30, Dec. 
YULE, G.U. (1927). On the method of investigating periodicities in disturbed series, with special reference to wolfer's sunspot numbers. Philosophical Transactions of the Royal Society of London - A, London, v.226, p.267-298. 


\section{Apêndice A - Função de Correlação}

A matriz de correlação, denotada por $R_{y y}(j)$, de um processo vetorial estocástico $y(k)$, com distribuição Gaussiana é definida por:

$$
R_{y y}(j)=E\left[y(k) y^{T}(k+j)\right]
$$

onde $E[$.$] indica o operador esperança.$

Em detalhes, a matriz de correlação de $y(k)$ é dada por:

$$
\begin{aligned}
& R_{y y}(j)= E\left[y(k) y^{T}(k+j)\right]= \\
&= {\left[\begin{array}{c}
y_{1}(k) \\
y_{2}(k) \\
\vdots \\
y_{n}(k)
\end{array}\right] } \\
& {\left[\begin{array}{llll}
y_{1}(k+j) & y_{2}(k+j) & \cdots & y_{n}(k+j)
\end{array}\right]=} \\
&= {\left[\begin{array}{cccc}
E\left[y_{1}(k) y_{1}(k+j)\right] & E\left[y_{1}(k) y_{2}(k+j)\right] & \cdots & E\left[y_{1}(k) y_{n}(k+j)\right] \\
E\left[y_{2}(k) y_{1}(k+j)\right] & E\left[y_{2}(k) y_{2}(k+j)\right] & \cdots & E\left[y_{2}(k) y_{n}(k+j)\right] \\
\vdots & \vdots & \vdots & \vdots \\
E\left[y_{n}(k) y_{1}(k+j)\right] & E\left[y_{n}(k) y_{2}(k+j)\right] & \cdots & E\left[y_{n}(k) y_{n}(k+j)\right]
\end{array}\right]=} \\
&= {\left[\begin{array}{cccc}
R_{y_{1} y_{1}}(j) & R_{y_{1} y_{2}}(j) & \cdots & R_{y_{1} y_{n}}(j) \\
R_{y_{2} y_{1}}(j) & R_{y_{2} y_{2}}(j) & \cdots & R_{y_{2} y_{n}}(j) \\
\vdots & \vdots & \vdots & \vdots \\
R_{y_{n} y_{1}}(j) & R_{y_{n} y_{2}}(j) & \cdots & R_{y_{n} y_{n}}(j)
\end{array}\right]=R(j) }
\end{aligned}
$$

onde os elementos de $R(j)$ do tipo $R_{y_{k} y_{l}}$, para $k \neq l$ são chamados de correlação cruzada de $y$, e para $k=l$ denominados de auto-correlação de $y$.

Para processos estacionários as seguintes propriedades são válidas: 


$$
\begin{aligned}
& R_{y y}(j)=E\left[y(k) y^{T}(k+j)\right] \\
& R_{y y}(-j)=E\left[y(k) y^{T}(k-j)\right] \\
& R^{T}{ }_{y y}(j)=E\left[y(k+j) y^{T}(k)\right] \\
& R^{T}{ }_{y y}(-j)=E\left[y(k-j) y^{T}(k)\right]
\end{aligned}
$$

onde $R_{y y}(-j)=R_{y y}{ }^{T}(j)$ e $R_{y y}(j)=R_{y y}^{T}(-j)$.

Por simplicidade de notação $R_{y y}(j)$ será denotado apenas por $R(j)$.

A função de correlação e a sua transformada de Fourier, denominada de densidade espectral de potência (PSD), medem o grau de dependência existente entre dois ou mais processos. 